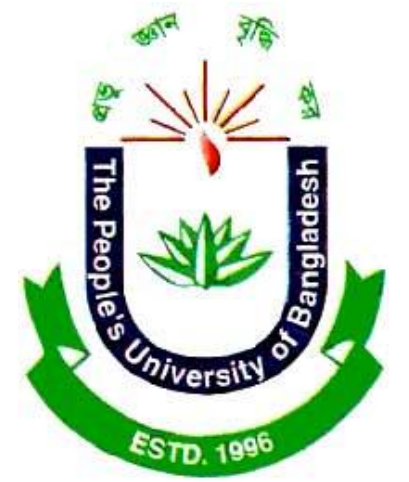

\title{
MIMO CHANNEL AND PERFORMANCE ANALYSIS USING OFDM SYSTEM FOR REDUCED BIT ERROR RATE \\ by
}

Saddam Hossain

ID No:10410203132

Md. Izaz Arefin Mrida

ID No: 12412203016

Pradip Chandra Das

ID No:10309203094

Abu Sayed

ID No:10410203424

Nahid Rahman

ID No: 10410203044

Supervisor: Ing. Md Mamunur Rashid

The thesis submitted to the faculty of Applied Science and Engineering in partial fulfillment of the requirements for the degree of Bachelor of Science in Electronics and Telecommunication Engineering

The People's University of Bangladesh (PUB)

Dhaka, Bangladesh

November 2014 


\section{THE PEOPLE'S UNIVERSITY OF BANGLADESH (PUB) \\ ELECTRONICS AND TELECOMMUNICATION ENGINEERING DEPARTMENT}

\section{MIMO CHANNEL AND PERFORMANCE ANALYSIS USING OFDM SYSTEM FOR REDUCED BIT ERROR RATE}

Approved by:

Ing. Md Mamunur Rashid

Chairman \& Assistant Professor

Dept. of Electronics \& Telecommunication Engineering 


\section{DECLARATION}

The work presented in the thesis is, to the best of our sense and faith, original and our own work except as acknowledged in the text. The material has not been submitted, either in whole or in part, for a degree at The People's University of Bangladesh or any other university but some materials have been included based on several sources in internet.

\section{Authors:}

Saddam Hossain

ID:10410203132

Md. Izaz Arefin Mrida

ID No: 12412203016

Pradip Chandra Das

ID:10309203094

Abu Sayed

ID: 10410203424

Nahid Rahman

ID: 1041020304
Signature

Signature

\section{Thesis Advisor:}

This thesis has been submitted for examination with my approval as a university advisor.

Ing. Md Mamunur Rashid

Chairman \& Assistant Professor

Dept. of Electronics \& Telecommunication Engineering

Signature 
MIMO Channel and Performance Analysis Using OFDM System for Reduced Bit Error Rate

Copyright@ 2014 by Saddam Hossain

All Rights Reserved. 
This thesis is dedicated to our parents and our honorable teacher Ing. Md Mamunur Rashid and those people who always support and love us in our academic career. 
"There is no scale to measure an erudite person, but wisdom."

-Saddam Hossain 


\begin{abstract}
Multiple-input Multiple-output (MIMO) systems use multiple antennas at the transmitter and receiver end, are a key technology to meet the growing demand for high data rate wireless systems. The aim of this thesis is to investigate MIMO system capacity with the aim of achieving optimum Bit Error Rate (BER) while increasing the system capacity using multicarrier delay diversity modulation (MDDM), proposed for fifth generation systems. In principle, the capacity of MIMO system can increase linearly with the number of antennas. Multiple antennas at the transmitter and receiver provide diversity in a fading environment. Furthermore, the research work in this thesis consists of different investigations of the basic principle of MIMO, Multiple-input Single-output (MISO) and Single-input Single-output (SISO) wireless communication systems with Space Time Codes (STC). A MISO systems and MIMO systems are schematized using MDDM which incorporated with Orthogonal Frequency Division Multiplexing (OFDM). OFDM is chosen over a single-carrier solution due to lower complexity of equalizers for high delay spread channels or high data rates. The design is implemented with binary Phase Shift Keying (BPSK) and simulated using MATLAB, which is examined in associated Additive White Gaussian Noise (AWGN) channel. The receiver-design is included with the maximal ratio combiner (MRC) receiving technique with perfect wisdom of channel state information (CSI). The theoretical performance is derived for AWGN channels and compared with the simulated results as well as compared between each system to another.
\end{abstract}




\section{ACKNOWLEDGEMENTS}

In the name of Allah, the beneficent, the merciful. Praise be to Allah, the lord of the universe. This report summarizes the achievements of the Bachelor Thesis work carried out between April and September 2014 at The People's University of Bangladesh, Dhaka, Bangladesh. We would like to express our deepest gratitude to our supervisor Ing. Md Mamunur Rashid, for his consistent encouragement and insightful advise. This thesis would not have been possible without his invaluable technical insight and continuous guidance. We would like to thank all our classmates in the Department of Electronics and Telecommunication Engineering for the past four years. Special thanks are dedicated to all of our teachers, friends and family who have been a source of encouragement and inspiration to accomplish this thesis. We would also like to deeply thank several students and researchers for providing us various sources (mention in references) that made this thesis possible.

Of course, We are also grateful to our parents because of their patience, continuous encouragement and love. Without them, this work would never have come into existence, and We would truly miss the phone calls, in which they asked for the status of our thesis work. 


\section{EXECUTIVE SUMMARY}

The incredible growth of mobile data and the use of smart-phones are creating unprecedented challenges for wireless service providers to overcome a global bandwidth shortage. As today's cellular providers attempt to deliver high quality, low latency video and multimedia applications for wireless devices, they are limited to a carrier frequency spectrum ranging between $700 \mathrm{MHz}$ and $2.6 \mathrm{GHz}$. The demand for high speed and reliable communication within the constraints of limited radio frequency spectrum and power, are the prime technical criteria for communication systems. Besides, to obtain a higher data rate at an acceptable bit error rate in larger bandwidth is required.

Obtaining this information is extremely important to deploy future generation cellular networks, it can be 5G. But there are few challenges to deploy 5G wireless communication systems in which sufficient bandwidth present. On the other hand, To mitigate severe fading channel conditions and bit error rate, a higher transmitted power level is required. Multiple-input multipleoutput (MIMO) communication systems have the potential to provide increased capacity and reliability without increasing the bandwidth or transmitted power. MIMO systems exploit time and spatial diversities by employing multiple antennas at the transmitter and receiver.

The main objective of this thesis is to investigate the fundamentals of MIMO systems with a multicarrier delay diversity modulation (MDDM) technique. A simple model was designed to incorporate MDDM in multiple-input singleoutput (MISO) and MIMO systems. The design is implemented with Binary Phase Shift Keying (BPSK) and simulated using MATLAB simulator, which is examined in an Additive White Gaussian Noise (AWGN) channel. The simulated performance results and theoretical analysis results were compared with the conventional single-input single-output (SISO) system results. The performance metric of bit error probability versus $E_{d} / N_{o}$ (energy per bit to noise power spectral density ratio) was used. To establish a fair comparison, the transmitted power for the SISO, MISO and MIMO systems was maintained equal. The results will show that the designed MISO and MIMO system perform within expected parameters of the theoretical analysis in the AWGN channel. 


\section{LIST OF ABBREVIATIONS}

MIMO

OFDM

BER

AWGN

MDDM

MISO

SNR

SISO

CSI

$\mathrm{MRC}$

STC

BPSK

QoS

IID

GRV

AoA

AoD

ToA

SCM

AHG

ADS
Multiple Input Multiple Output

Orthogonal Frequency Division Multiplexing

Bit Error Rate

Additive White Gaussian Noise

Multicarrier Delay Diversity Modulation

Multiple Input Single Output

Signal-to-Noise Ratio

Single Input Single Output

Channel State Information

Maximal Ratio Combiner

Space Time Codes

Binary Phase Shift Keying

Quality of Service

Independent Identically Distributed

Gaussian Random Variables

Angle of Arrival

Angle of Departure

Time of Arrival

Spatial Channel Model

Ad-Hoc Group

Azimuth-Delay Spread 


$\begin{array}{ll}\text { DFT } & \text { Discrete Fourier Transform } \\ \text { STBC } & \text { Space-Time Block Codes } \\ \text { STTC } & \text { Space-Time Turbo Trellis Codes } \\ \text { BLAST } & \text { Bell Labs layered space-time technology } \\ \text { FEC } & \text { Forward Error Correction } \\ \text { ISI } & \text { Inter-symbol Interference } \\ \text { IDFT } & \text { Inverse Discrete Fourier Transform } \\ \text { FFT } & \text { Fast Fourier Transform } \\ \text { IFFT } & \text { Inverse Fast Fourier Transform } \\ \text { FDM } & \text { Frequency Division Multiplexing } \\ \text { LOS } & \text { Line of Sight } \\ \text { A/D } & \text { Analog to Digital } \\ \text { D/A } & \text { Digital to Analog }\end{array}$




\section{LIST OF FIGURES}

Figure 2.1: MIMO system

Figure 2.2: SIMO channel environment

Figure 2.3: Multi-path signals in the multiple antenna elements

Figure 2.4: Comparison of mean capacity of SISO, SIMO and MISO systems as a function of SNR.

Figure 2.5: Increasing the MIMO capacity with increasing number of antennas

Figure 3.1: SISO system

Figure 3.2: SIMO system

Figure 3.3: MISO system

Figure 3.4: MIMO system

Figure 3.5: Block diagram of OFDM modulation using IFFT

Figure 3.6: Comparison of bandwidth efficiency between FDM and OFDM

Figure 3.7: The addition of the cyclic guard interval

Figure 3.8: Diagram of the concept of cyclic prefix

Figure 3.9: MRC for BPSK with time diversity

Figure 3.10: MRC for BKSP for Space Diversity

Figure 4.1: Block diagram of MDDM transmitter

Figure 4.2: OFDM modulator

Figure 4.3: Block diagram of MDDM receiver

Figure 4.4: OFDM demodulator

Figure 5.1: BPSK correlation demodulator for MIMO system with MDDM.

Figure 5.2: Simulation of MDDM MIMO system in AWGN

Figure 5.3: Simulation result of MDDM MISO system in AWGN

Figure 5.4: Simulation result of MDDM-2*2MIMO system in AWGN ..... Figure 5.5: Simulation result of MDDM 2*3-MIMO system in AWGN...... Figure 5.6: Simulation result of MDDM $3 * 3-$ MIMO system in AWGN Figure 5.7: Simulation result of all MDDM MIMO systems in AWGN

\section{LIST OF TABLES}

Table 1: The BPSK modulated symbol scheme

Table 2: Demodulation of BPSK signal 


\section{Contents}

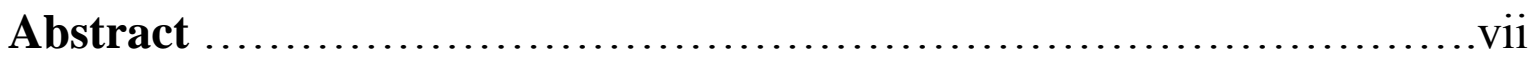

Acknowledgements ................................................

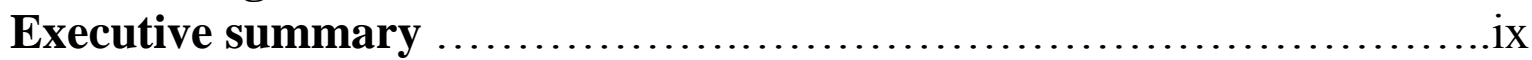

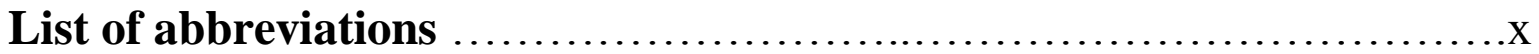

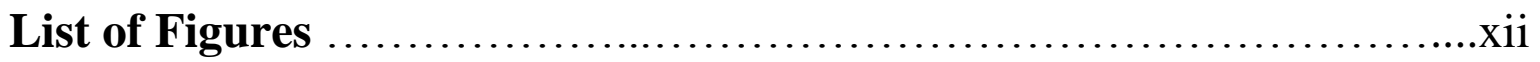

List of Tables .........................................................

\section{Introduction}

1.1 Background and motivation....

1.2 Research problems and objectives

1.3 Aims of this thesis.

1.4 Thesis organization

\section{MIMO system background}

2.1 Literature review.

2.2 MIMO system model.

2.3 MIMO channel modeling

2.4 Statistical MIMO model

2.5 Antenna diversity.

2.6 Measurement.

2.7 Capacity analysis

2.7.1 Channel matrix normalization

2.7.2 SISO system capacity....

2.7.3 SIMO and MISO system capacity

2.7.4 MIMO system capacity.

\section{MIMO systems and multicarrier delay diversity}

3.1 MIMO Channel Model Classification.

3.1.1 Single-Input Single-Output System. ...

3.1.2 Single-Input Multiple-Output System....

3.1.3 Multiple-Input Single-Output System.

3.1.4 Multiple-Input Multiple-Output System.

3.2 Space Time Coding....

3.3 Multicarrier delay diversity in MIMO systems

3.4 Orthogonal frequency division multiplexing

3.4.1 Generation of OFDM

3.4.2 Cyclic guard interval

3.5 The Multipath and Fading Channel 
3.5.1 Flat Rayleigh Fading Channel

3.5.2 Maximal-Ratio Combining

3.6. Summary.

\section{Multicarrier delay diversity modulation transmitter and receiver models for MIMO systems}

4.1 The multicarrier delay diversity modulation scheme

4.2 MDDM transmitter

4.2.1 Binary information source and $M$ PSK modulator

4.2.2 OFDM Modulator.

4.2.3 Cyclic delay addition

4.2.4 Guard interval (Cyclic Prefix) addition

4.3 Digital to analog conversion and RF modulation

4.4 MDDM receiver.

4.5 Summary

\section{Analysis and simulation of multicarrier delay diversity modulation scheme}

5.1 Simulation of MDDM transmitter

5.2 Simulation of MDDM receiver.

5.3 Simulation and performance analysis of MDDM in AWGN channel

5.3.1 Performance analysis of SISO and MISO system with two transmitting and one receiving antenna.

5.3.2 Performance analysis of SISO and MIMO system with two transmitting and two receiving antennas

5.3.3 Performance analysis of SISO and MIMO system with two transmitting and three receiving antennas 5.3.4 Performance Analysis of SISO and MIMO System with three transmitting and three receiving antennas

Conclusion

Appendix

References 


\section{Chapter 1}

\section{INTRODUCTION}

One of the challenges faced by future wireless communication systems is to provide high data rates at high quality of service (QoS). Combined with the fact that spectrum is a scarce resource and propagation conditions are hostile this requires radical increase in spectral efficiency and link reliability. The system requirements can be met by the combination of two powerful technologies in the physical layer design: multi input and multi output (MIMO) antennas and orthogonal frequency division multiplexing (OFDM) modulation [1]. These two are considered to be the key enabling technologies that will help the next generation networks exceed the current system performance. Use of multiple antennas can offer significant increase in data throughput, spectral efficiency and link range without additional bandwidth or transmit power [2]. Link reliability and diversity are improved but this also increases frequency selective fading in the channels. The primary advantage of OFDM is its ability to cope with severe channel conditions like frequency selective fading due to multipath propagation, attenuation of high frequency in long copper wire and narrowband interference [2]. Multicarrier Delay Diversity Modulation(MDDM) technique has been recognized as the MIMO technique to increase transmission capacity. Therefore current research is trying to find well performing less complex detection algorithms for AWGN channel models with fading and no fading channel. Simulated performance of MIMO is strongly influenced by the choice of the channel model. Modeling of the radio channel is essential for system design and performance evaluation [3]. Inaccurate channel model selected for MIMO-OFDM can lead to suboptimal transmissions and incorrectly assessed performance of the system [3]. Channel conditions like spatial correlation have been noticed to substantially impair the performance of MIMO wireless communication systems. Most of the research in MIMO OFDM performance has been concentrated on using independent and identically distributed Rayleigh channel model which will also be considered along with more realistic channel models with spatial correlation. The overall goal of this work is to evaluate and improve the performance of MIMO OFDM system with Delay Diversity Modulation(MDDM). 


\subsection{Background and motivation}

Currently we are witnessing the deployment of fourth generation (4G) mobile communication systems which are expected to outperform third generation (3G) systems in terms of supported data rates and technologies. Despite the fact that the $4 \mathrm{G}$ systems can offer high data rates as subscribers demand, they may not be sufficient to meet the requirements for future high data rate applications. New multimedia applications such as video streaming and wireless teleconferencing require higher data rate communications. New activities in various organizations have already started researching for fifth generation $(5 \mathrm{G})$ mobile wireless communication systems in order to cope with these increasing demands in data rates. It is expected that the $5 \mathrm{G}$ systems will support data rates up to $1 \mathrm{~Gb} \mathrm{Mb} / \mathrm{s}$ for mobile applications [4].

From this information, one of the major challenges facing modern communications is to satisfy the ever increasing demand of high speed reliable communications with the constraints of extremely limited frequency spectrum and limited power. Wireless communications systems like cellular mobile communications, internet and multimedia services require very high capacity to fulfill the demand of high data rates, Multiple-input multipleoutput (MIMO) wireless systems may keep important role. Multiple-input multiple-output (MIMO) wireless systems, characterized by multiple antenna elements at the transmitter and receiver, have shown astonishing increase in spectral efficiency and significant improvement in link reliability in rich multipath environments. One possible choice for the $5 \mathrm{G}$ systems is to utilize the MIMO technology in order to achieve high spectral efficiency and consequently provide reliable high data rates. While coding and signal processing are key elements to the successful implementation of MIMO systems, the propagation channel, the antenna design and the accuracy of measurement data represent major design parameters that ultimately impact MIMO system performance. Understanding the effects of these parameters on MIMO systems performance is essential for the successful design and deployment of MIMO systems and this is the motivation behind this thesis work.

On the other hand, another important motivation behind this thesis work is the wireless communication systems with multiple transmit and multiple receive antennas can provide high capacity at low probability of bit error with extremely low power, even in dense scattering and multipath environments. These multiple-input multiple-output (MIMO) systems with appropriate space-time codes have been an area of recent research as they hold the promise of ever increasing data rates. The capacity of a MIMO system can be 
increased linearly by increasing the number of transmit and receive antennas. The applications of MIMO systems in a frequency selective channel require equalization and other techniques to compensate for frequency selectivity of the channel, which add to the complexity of these systems. In recent years, orthogonal frequency division multiplexing (OFDM) has been widely used in communications systems to operate in frequency selective channels including several wireless communication standards. Communication systems with a MIMO-OFDM combination can significantly improve capacity and reliability by exploiting the robustness of OFDM to fading, enhanced by adding more diversity gain via space time codes. In this research, delay diversity in combination with a MIMO-OFDM system is the primary focus and is referred to as multicarrier delay diversity modulation (MDDM).

\subsection{Research problems and objectives}

The promising advantages of MIMO systems over traditional single antenna systems depend largely on the correlation properties between antenna elements [5]. Low correlation values reveal high MIMO system performance in terms of data rates and link reliability and high correlation values indicate the opposite. There are several factors affecting the correlation properties. Among these factors are the environment physical parameters [6], the antenna array configuration and the antenna element properties. These parameters play a key role in determining the MIMO system performance.

Despite the fact that MIMO systems take the spectral efficiency to a new level, there are challenges accompanying this system. One of the main challenges is the computational complexity of the receiver, which increase with number of antennas employed. This is due to the fact that independent data streams transmitted from each transmit antenna occupy the same frequency spectrum and therefore, interfere with one another in addition to the effects of system related noise. Hence, the challenge is to develop MIMO receiver (MIMO MDDM receiver) which has high performance and, simultaneously, has low and manageable computational complexity. In this regard, this thesis focuses on the development of the MIMO computational complexity and performance which is optimal MIMO-BER and increase channel capacity.

One of the objectives of this thesis work is to conduct a thorough investigation of the effect of propagation environment characteristics, antenna array configuration properties on MIMO system performance. Successful design and deployment of MIMO wireless communication systems require detailed channel characterization. 
In general, the objective of this thesis is to develop a MIMO-OFDMA system with MDDM scheme which has near optimal BER with much reduced computational complexity and the best channel performance.

Specific Objectives Specifically the aim of this thesis is to:

Extensively study the available MIMO-MDDM schemes, analyze and evaluate the computational complexities of these schemes in conjunction with their performances under AWGN channel conditions and antenna configurations.

Analyze and investigate the best combination of MDDM schemes considering the computational complexity and BER.

Evaluate and compare the BER performance and throughhput enhancement of the proposed scheme with the existing detection techniques.

The main objective of this thesis is to investigate the fundamentals of MIMO systems with a multicarrier delay diversity modulation (MDDM) technique.

A simple model was designed to incorporate MDDM in multiple-input singleoutput (MISO) and MIMO systems. The design is implemented with Binary Phase Shift Keying (BPSK) and simulated using MATLAB simulator, which is examined in an Additive White Gaussian Noise (AWGN) channel.

Finally Analysis The simulated performance results and theoretical analysis results, were compared with the conventional single-input single-output (SISO) system results.

\subsection{Aims of this thesis}

The goal of this thesis is to investigate MIMO-OFDM system capacity with the aim of achieving optimum Bit Error Rate (BER) while increasing the system capacity using multicarrier delay diversity modulation (MDDM), tested in AWGN channel.

\subsection{Thesis organization}

This thesis is organized in five chapters. Chapter I describes the background and motivation of this thesis. Furthermore, refers research problems and objectives as well as aims of this thesis.

Chapter II introduces the literature review, referred in previous work by other researchers, which forms the foundation of this thesis. Such as MIMO channel modeling, antenna diversity, channel measurement and capacity analysis of SISO, SIMO, MISO and MIMO system. 
Chapter III introduces the fundamentals of MIMO systems and the multicarrier delay diversity modulation scheme. It discusses the capacity of MIMO systems, space time coding and use of time delay diversity as a space time coding technique in MIMO systems.

Chapter IV represents the modeling and simulation of the MDDM transmitter and receiver, OFDM modulator scheme, digital to analog(D/A) conversion , RF modulation, cyclic delay addition and guard interval (Cyclic Prefix) addition

Chapter $\mathrm{V}$ analyzes the performance of this modulation technique in an AWGN channel with AWGN. Chapter V reviews the summary of the work done. Appendix.

In Chapter five simulation results of all the detection schemes considered in Chapters three and four are provided. Most of the simulation results are BER versus SNR characteristics, which are used to compare performance of different MIMO detection schemes, while there are also complexity and throughput comparisons. 


\section{Chapter 2}

\section{MIMO SYSTEM BACKGROUND}

\subsection{Literature review}

The literature review outlines previous work by other researchers, which forms the foundation of this thesis. These topics are organized in the following order:

\subsection{MIMO system model}

A multiple-input multiple-output (MIMO) system with multiple $(L)$ transmit antennas and multiple $(J)$ receive antennas is illustrated in Figure 2.1. MIMO systems are the focus of this thesis. Therefore, the MIMO model is discussed in detail in chapter 3 . The representation of the model is largely based on [1, 8]. For a faded channel, it is assumed that channel responses from each transmit antenna to each receive antenna are independent.

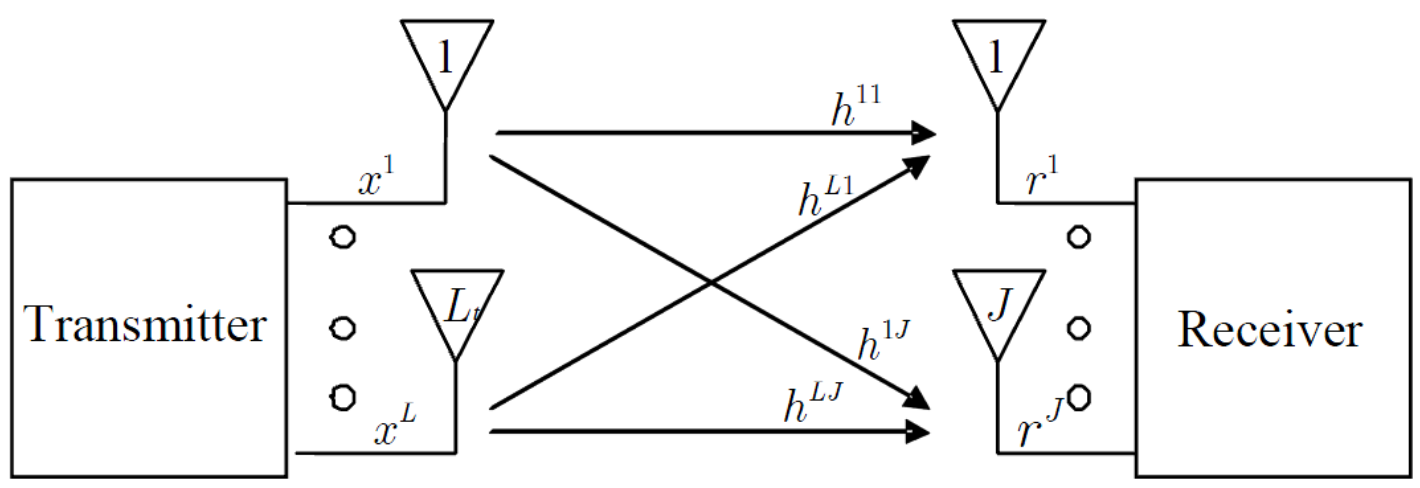

Figure 2.1: MIMO system

$$
\begin{aligned}
& r^{j}=h^{1 j} x^{1}+h^{2 j} x^{2}+\cdots+h^{L j} x^{L}+n^{j} \\
& {\left[\begin{array}{c}
r^{1} \\
r^{2} \\
\vdots \\
\vdots \\
r^{J}
\end{array}\right]=\left[\begin{array}{cccc}
h^{11} & h^{21} & \ldots & h^{L 1} \\
h^{21} & h^{22} & \ldots & h^{L 2} \\
\vdots & \vdots & \vdots \\
\vdots & \vdots & \vdots \\
h^{J 1} & h^{J 2} & \ldots & h^{J L}
\end{array}\right]\left[\begin{array}{c}
x^{1} \\
x^{2} \\
\vdots \\
\vdots \\
x^{L}
\end{array}\right]+\left[\begin{array}{c}
n^{1} \\
n^{2} \\
\vdots \\
\vdots \\
n^{J}
\end{array}\right]}
\end{aligned}
$$




$$
r=\boldsymbol{H} x+n
$$

where $\mathbf{r}$ and $\mathbf{n}$ represent the $J \times 1$ received signal and noise column vectors, $\mathbf{H}$ is a

$J \times L$ complex channel matrix and $\mathbf{x}$ is the $L \times 1$ transmitted column matrix. [86] All the elements in the channel matrix are considered independent identically distributed (IID) complex Gaussian Random Variables (GRVs), and similarly, the elements of the noise vector are also complex Gaussian random variables.

\subsection{MIMO channel modeling}

Channel modeling is a fundamental research area in wireless communication due to the complexity of the signal propagation process. It plays a crucial role in the investigation of MIMO system capacity.

The independent and identically distributed (i.i.d.) channel model has been used by researchers to derive the useful bounds of MIMO capacity [7]. As a stochastic channel model, the i.i.d. channel is the simplest and the most common channel model. Many different MIMO channel models have been presented in previous work [8]. MIMO channel models can be classified in many different ways. The MIMO channel models can be divided into wideband and narrowband by considering the system bandwidth [8]. In the wideband channel models, the channel is assumed to have different channel responses for different frequencies, so that the channel is a frequency selective fading channel. On the contrary, the narrowband channel model is assumed to have an equal response over the system bandwidth. By considering the characteristics of the channel parameters, the MIMO channel models can be divided into non-physical and physical models [8]. The nonphysical models use non-physical channel parameters based on the channel's statistical characteristics. However, the physical model uses some crucial physical parameters, such as Angle of Arrival (AoA), Angle of Departure (AoD) and Time of Arrival (ToA) to model the channel. examples of physical narrowband channel models are 'One-Ring' and SCM channel models.

The 'One-Ring' channel model is one of the remarkable MIMO channel model. As compared to i.i.d. channel model, the 'One-Ring' channel model takes into account the inter-element distance which makes it possible to study mutual coupling and correlation effects [9]. 
The SCM (Spatial Channel Model) is an example of a more realistic channel model [10]. It is a standardized model developed by 3GPP-3GPP2 spatial channel model (SCM) ad-hoc group (AHG), which are standardization bodies for $3 \mathrm{G}$ cellular systems [10]. This model allows us to perform system level simulations, and offers three propagation scenarios for investigation: suburban macro-cell, urban macro-cell and urban micro-cell [10].

Transfer matrix modeling is the another simplest channel models directly compute the channel matrix $\mathbf{H}$ based upon a statistical description. For example, in a NLOS propagation scenario, it is commonly assumed that the channel between one transmit and one receive antenna will have a magnitude and phase that follow Rayleigh and uniform distributions, respectively [11]. This combination indicates that the individual complex elements of $\mathbf{H}$ are circular symmetric complex Gaussian random variables. In this case, the distribution is completely specified by the full channel correlation matrix $R_{H}=E\left\{h h^{H}\right\}$, where $\mathrm{h}=\operatorname{vec}(\mathrm{H})$ and $\operatorname{vec}($.$) stacks the columns of the matrix$ argument into a single column vector. In case of no correlation between the signals on different antennas, the full channel correlation matrix $R_{H}$ is an identity matrix, i.e. $R_{H}=\mathrm{I}$, which leads to independent matrix entries. This is the case when orthogonal channels are assumed. However, if the correlation structure is to be included, a correlation matrix must be constructed directly from measured data [12] or from a correlation model [13]. If the fading statistics at the transmit and receive sides are assumed to be independent, a separable correlation structure, referred to as the Kronecker product model [40-43], can be created. The Kronecker structure is in the form $\boldsymbol{R}_{\boldsymbol{H}_{\text {kron }}}=$ $\boldsymbol{R}_{t x} \otimes \boldsymbol{R}_{\boldsymbol{r} x}$ where $\mathbf{R}_{t x}$ and $\mathbf{R}_{r x}$ are correlation matrices for signals on the transmit and receive arrays, respectively, and $\otimes$ denotes the Kronecker product. Utilizing the Kronecker model, a channel matrix $\boldsymbol{H}_{\boldsymbol{k} \text { ron }}$ can be generated according to

$$
\boldsymbol{H}_{\text {kron }}=\boldsymbol{R}_{\boldsymbol{r x}}{ }^{1 / 2} \boldsymbol{H}_{\boldsymbol{w}}\left(\boldsymbol{R}_{\boldsymbol{t x}}^{1 / 2}\right)^{T}
$$

This approach is very simple to implement, and therefore facilitates assessment of space-time codes using Monte Carlo simulation approaches. Some studies have shown that this model is highly effective in matching measured results for systems with up to four antenna elements [12]. However, recent work has demonstrated key deficiencies in this Kronecker product model [13]. In fact, one study has demonstrated that the Kronecker structure leads to high errors not only in the computed capacity but also in the 
correlation matrix representation [46]. Another study has confirmed this error in the capacity as well as error in the joint statistics of the resulting transfer matrix [14]. Nevertheless, the simplicity of this model makes it an attractive starting point in the analysis of any MIMO system performance [15]. Therefore, improved modified versions were proposed in [12-16].

Multipath characterization is an another approach for modeling MIMO wireless channels is to directly describe the properties of the physical channel multipath components. In this approach the obtained multipath channels are independent of the properties of the associated antenna system which facilitates studying the impact of antenna properties. Models capturing multipath behavior range from deterministic site-specific ray-tracing to simpler statistical descriptions.

1. Deterministic ray-tracing. Deterministic ray-tracing modeling begins by creating a two dimensional (2D) or three dimensional (3D) geometrical model of the propagation environment. Then the response of the model to electromagnetic excitation is computed through computational techniques. Such models can also provide statistical channel information by applying Monte Carlo analysis on many random transmit/receiver locations and/or model geometries. Ray-tracing techniques based on geometrical optics, often supplemented by diffraction theory to enhance accuracy in shadow regions, have emerged as the most popular techniques for analyzing site-specific scenarios due to their ability to analyze very large structures with reasonable computational resources [17-18]. Several investigations of MIMO systems performance have been conducted based on ray-tracing techniques [19]. Raytracing techniques have demonstrated reasonable accuracy in predicting largescale path loss variation. However, preliminary comparisons of ray tracing predications with measurements indicate that the simulations tend to underestimate MIMO channel capacity [20]. This likely due to over simplification of the geometrical scenario representation than failure of the electromagnetic simulation approach. Other recent work [21] has shown promising agreement between measured and simulated results of angle of arrival (AOA) estimation in microcells.

2. Geometric scattering models. The high computational cost of rigorous raytracing simulations in addition to the fact that this type of technique is very site specific are the main drawbacks of ray-tracing techniques. Due to that more approximate models have appeared where more simplified geometries and scattering mechanisms are assumed [22-23]. Scatterers are modeled as 
discrete objects located around the receiver and/or transmitter. These objects can represent site-specific obstacles, or their locations and scattering properties (e.g. cross-section) which can be defined in terms of their statistical distribution. These models also allow for dynamic channel evolution by computing the response as the transmitter or receiver moves through the environment.

3. Statistical scattering models. Statistical cluster models directly specify distributions on the multipath angle of departure (AOD), AOA, time of arrival (TOA) and amplitude by grouping multipath components into clusters that decay exponentially with increasing delay time [24]. A single cluster might correspond to a single scattering object. In more advanced models, the birth and death of clusters due to movement of the subscriber can be taken into account [25]. Statistical descriptions of the multipath arrival parameters have been obtained through measurements [26] and ray-tracing. In [27] cluster and discrete scattering models are combined to include both distant and local scattering.

\subsection{Statistical MIMO model [28]}

Recall that delay spread and Doppler spread are the most important factors to consider in characterizing the SISO system. In the MIMO system which employs multiple antennas in the transmitter and/or receiver, the correlation between transmit and receive antenna is an important aspect of the MIMO channel. It depends on the angle-of-arrival (AoA) of each multipath component. Consider a SIMO channel with a Uniform Linear Array (ULA) in which $M$ antenna elements are equally spaced apart, with an inter-distance of $\mathrm{d}$ as shown in Figure 2.2. Let $y_{i}(t)$ denote a received signal at the $\mathrm{i}^{\text {th }}$ antenna element with the channel gain $\alpha_{i}$, delay $\tau_{i}$, and angle of arrival (AoA) $\phi_{i}$. As shown in Figure 2.3, the AoA is defined as the azimuth angle of incoming path with respect to the broadside of the antenna element. Note that the received signal of each path consists of the enormous number of irresolvable signals received around the mean of AoA in each antenna element. A vector of the received signals $y(t)=\left[y_{1}(t), y_{2}(t), \ldots \ldots y_{M}(t)\right]^{T}$ in the uniform linear array (ULA) of M elements can be expressed as 


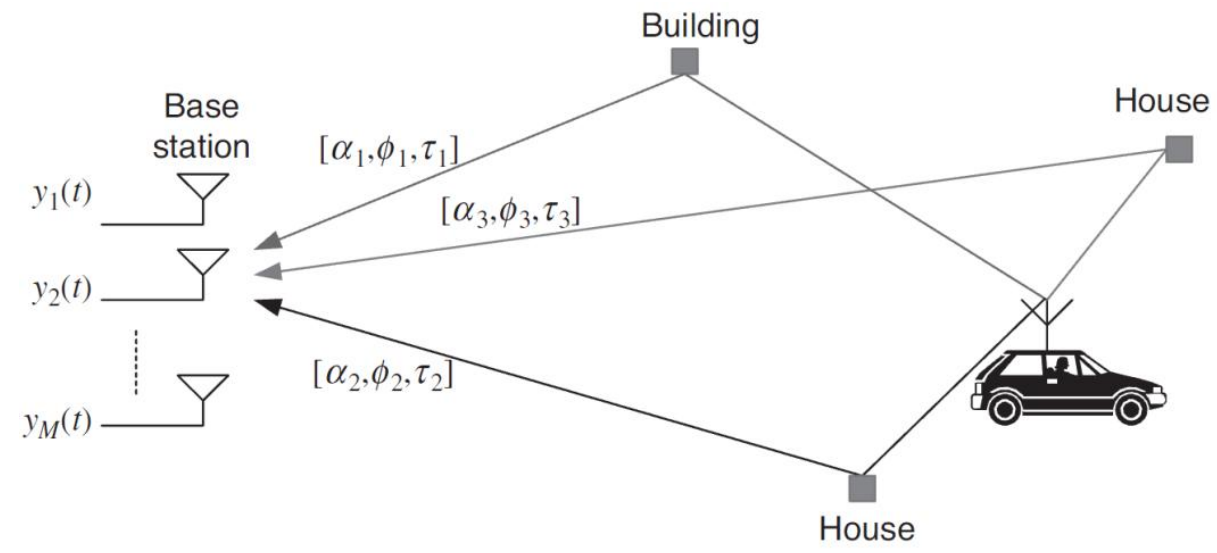

Figure 2.2: SIMO channel environment [28]

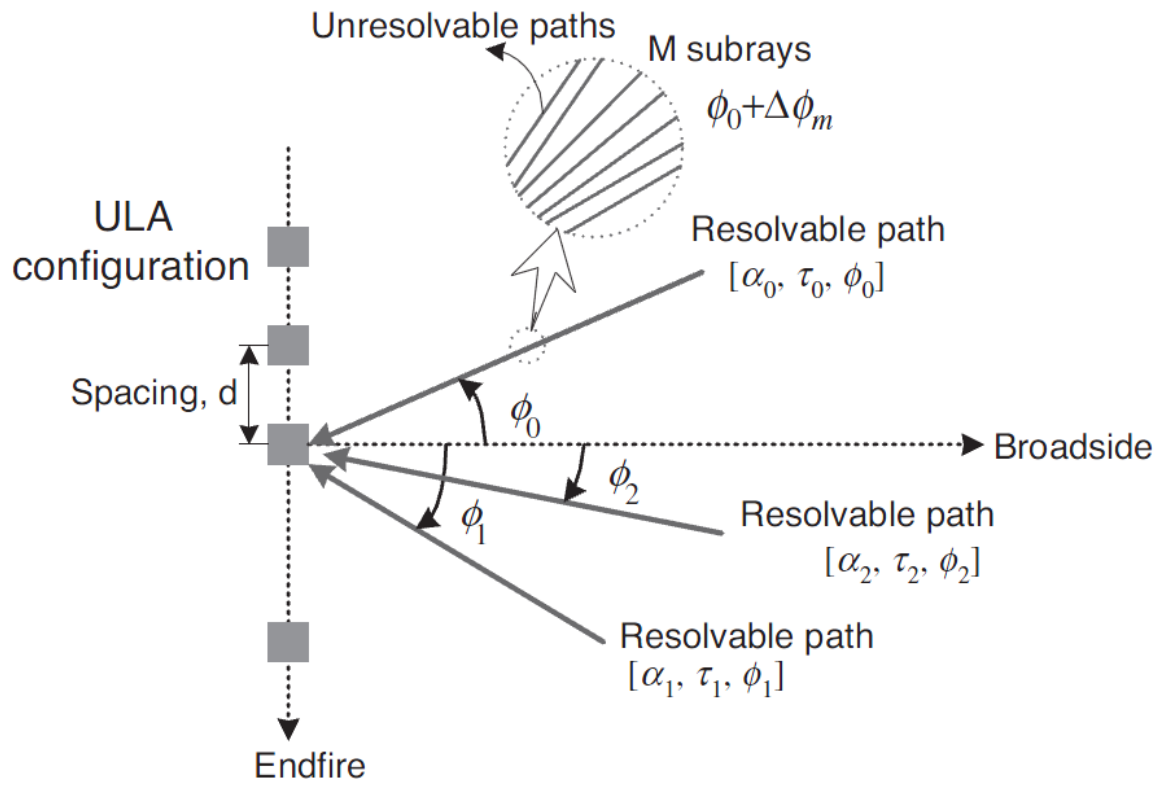

Figure 2.3: Multi-path signals in the multiple antenna elements [28]

$$
y(t)=\sum_{i=1}^{l} \alpha_{i} \boldsymbol{c}\left(\phi_{i}\right) x\left(t-\tau_{i}\right)+N(t)
$$

where I denotes the number of paths in each antenna element and $c\left(\phi_{i}\right)$ is an array steering vector. The array steering vector is defined as

Where

$$
\boldsymbol{c}\left(\phi_{i}\right)=\left[c_{1}(\phi), c_{1}(\phi), \ldots \ldots c_{M}(\phi)\right]^{T}
$$

$$
c_{m}(\phi)=f_{m}(\phi) e^{-j 2 \pi(m-1)(d / \lambda) \sin \phi}, \quad m=1,2, \ldots ., M
$$


In Equation (2.4), $f_{m}(\phi)$ denotes a complex field pattern of the $m^{\text {th }}$ array element and $\lambda$ is the carrier wavelength. The received signal in Equation (2.3) can be expressed in the following integral form:

$$
y(t)=\iint c(\phi) h(\phi, \tau) x(t-\tau) d \tau d \phi+N(t)
$$

where $h(\phi, \tau)$ represents a channel as a function of ADS (Azimuth-Delay Spread).

\subsection{Antenna Diversity [28]}

We have mentioned that the multiple antenna techniques can be broadly classified into two categories: spatial multiplexing techniques or diversity techniques. In this section, we will study the basic concepts of antenna diversity techniques. For AWGN channel, the slope of BER versus SNR curve for AWGN channel goes to infinity as SNR becomes large, that is, showing a water-falling type of BER performance as SNR increases. The fundamental goal of the antenna diversity techniques is to convert an unstable time-varying wireless fading channel into a stable AWGN-like channel without significant instantaneous fading, thereby steepening the BER versus SNR curve. MIMO antenna diversity techniques are used to mitigate degradation in the error performance due to unstable wireless fading channels, for example, subject to the multipath fading. Diversity in data transmission is based on the following idea: The probability that multiple statistically independent fading channels simultaneously experience deep fading is very low. There are various ways of realizing diversity gain, including the following ones:

Space diversity: sufficiently separated multiple antennas are used to implement independent wireless channels.

Polarization diversity: independent channels are implemented using the fact that vertically and horizontally polarized paths are independent.

Time diversity: same information is repeatedly transmitted at sufficiently separated (more than coherence time) time instances.

Frequency diversity: same information is repeatedly transmitted at sufficiently separated (more than coherence bandwidth) frequency bands.

Angle diversity: multiple receive antennas with different directivity are used to receive the same information-bearing signal at different angles. 


\subsection{Measurement [29]}

Transfer matrix measurement is the most straightforward approach to characterize MIMO wireless channels is to deploy a system that directly measures the $N_{r}$ and $N_{t}$ channel matrix. In this case, all components in the channel part are embedded in the measured channel and the measurements will only be applicable for the analysis of systems employing the same array configurations and antenna elements. Results based on a variety of measurement campaigns have appeared in literature, the reported results are usually in terms of channel capacity, signal correlation structure, rank of channel matrix, path loss and delay spread. The measurement campaigns can be classified based on the architecture of the measurement equipment into two main groups.

1. Measurement-based on true array system. This type of measurement equipment uses a true array system where all antennas operate simultaneously. The main advantages of such systems are the closeness to real world MIMO channels and ability of measuring channels that vary in time. However, the cost of the parallel transmit and receive electronics is the main drawback of this type of measurement equipments.

2. Measurement-based on switched array or virtual array. Switched array designs use a single transmitter and single receiver to measure the transfer function with high speed switches sequentially connecting all array elements to the electronics. Switching times for such systems are generally very low (few $\mu$ s to $100 \mathrm{~ms}$ ), indicating that the measurement over all antenna pairs can be conducted before the channel changes appreciably for most environments of practical interest. Virtual array instruments use precision displacement (or rotation) of a single antenna element to prescribed locations. A complete channel matrix measurement often takes several seconds or minutes, requiring a long mean stationary time of the measured channel. Therefore, virtual arrays are most suitable for fixed indoor measurement campaigns when activity is low. Measurement impairments such as thermal additive noise and PN in the local oscillators are other drawbacks of such systems.

\subsection{MIMO Capacity Analysis [28]}

Compared to a conventional single antenna system, the channel capacity of a multiple antenna system with $\mathrm{N}_{\mathrm{T}}$ transmit and $\mathrm{N}_{\mathrm{R}}$ receive antennas can be increased by the factor of $\left(\mathrm{N}_{\mathrm{T}}, \mathrm{N}_{\mathrm{R}}\right)$, without using additional transmit power or spectral bandwidth. Due to the ever increasing demand of faster data transmission speed in the recent or future telecommunication systems, the 
multiple antenna systems have been actively investigated and successfully deployed for the emerging broadband wireless access networks (e.g., Mobile WiMAX) [212]. Even when a wireless channel with high channel capacity is given, we still need to find good techniques to achieve high speed data transmission or high reliability. Multiple antenna techniques can be broadly classified into two categories: diversity techniques and spatial-multiplexing techniques. The diversity techniques intend to receive the same information bearing signals in the multiple antennas or to transmit them from multiple antennas, thereby improving the transmission reliability. A basic idea of the diversity techniques is to convert Rayleigh fading wireless channel into more stable AWGN-like channel without any catastrophic signal fading. We addressed the diversity techniques in section 2.5. In the spatial-multiplexing techniques, on the other hand, the multiple independent data streams are simultaneously transmitted by the multiple transmit antennas, thereby achieving a higher transmission speed. However, when the diversity techniques are used, the achievable transmission speed can be much lower than the capacity of the MIMO channel.

Claude Shannon first derived the channel capacity for additive white Gaussian noise (AWGN) channels in 1948 [30]. Compared with the scalar AWGN channels, a MIMO system can offer significant improvement to either communication quality (bit-error rate or BER)or transmission date rate (bits/sec) by exploiting spatial diversity [31]. We summarize absolute capacity bounds, which compare SISO, single-input-multiple-output (SIMO) and multiple-input single-output (MISO) capacities. Since feedback is an important consideration of communication system design, we further discuss a more specific case which takes into account a prior knowledge of the channel matrix at the transmitter. Before discussing capacity, some assumptions need to be stated:

- In all these cases, we focus on the single user form of capacity, so that the received signal is corrupted by additive white Gaussian noise only.

- Capacity analysis is based on a "quasi-static" situation which means that the channel is assumed fixed within a period of time (a burst), and the burst is assumed to be a long enough duration in which sufficient bits are transmitted to make information theory be meaningful [31].

- The channels are assumed to be memory-less channels which means that each channel realization is independent of the others [32].

\subsubsection{Channel Matrix normalization [29]}


Since the MIMO system performance depends on both the channel correlation properties and the average receive SNR, it is important and convenient to properly normalize the channel matrix for correct interpretation of the results. For a given channel matrix $\mathrm{G}$, its normalized version $\mathrm{H}$ can be obtained as follows

$$
H=\frac{1}{\alpha} G
$$

where $\alpha$ is a normalization factor given by

$$
\alpha=\sqrt{\frac{1}{N_{r} N_{t}}\|G\|_{F}^{2}}
$$

where $\|$.$\| denotes the Frobenius norm of the matrix argument. With$ normalized channel matrix, the average receive SNR can be defined in terms of the total transmitted signal power $\sigma_{x}^{2}$ and noise power at each receive antenna $\sigma_{n}^{2}$ as

$$
\rho=\frac{\sigma_{x}^{2}}{\sigma_{n}^{2}}
$$

It is worthy to notice that there are different normalization techniques used in MIMO literature. The above normalization technique keeps the total power in each channel realization fixed but does not remove the power imbalance between the elements of the channel matrix. In it is shown that the power imbalance results in significant MIMO system performance degradation even at low correlation propagation environment. Compensating the power imbalance requires a normalization technique that operates on each subchannel. In this thesis the issue of power imbalance is not considered.

\subsubsection{SISO System Capacity}

For a SISO (Single-Input-Single-Output) system the capacity is given by [32]

$$
C_{\text {SISO }}=\log _{2}\left(1+\rho|h|^{2}\right) \quad \text { bps } / H z
$$

where $\mathrm{r}$ is the SNR at receiver antenna, and $h$ is the normalized complex gain of the channel.

\subsubsection{SIMO and MISO System Capacity}

With $N R$ RX antennas, the single-input-multiple-output (SIMO) system capacity is 


$$
C_{M I S O}=\log _{2}\left(1+\rho \sum_{m=1}^{N_{r}}\left|h_{m}\right|^{2}\right) \quad b p s / H z
$$

where $h_{m}$ is the gain for ; $m^{\text {th }} \mathrm{R}_{\mathrm{X}}$ antenna.

However, if $N_{T} \mathrm{~T}_{\mathrm{X}}$ antennas are used, a multiple-input-single-input (MISO) is achieved. The capacity is given [32]

$$
C_{\text {MISO }}=\log _{2}\left(1+\frac{\rho}{N_{T}} \sum_{n=1}^{N_{T}}\left|h_{n}\right|^{2}\right) \quad \text { bps } / H z
$$

where $h_{n}$ is the gain for $n^{\text {th }}$ TX antenna. In order to ensure transmitter power restriction, SNR is normalized by $N T$. Figure 2.4 illustrates the capacity comparison of SISO, SIMO and MISO system versus SNR using Equation (2.9) - Equation (2.11).

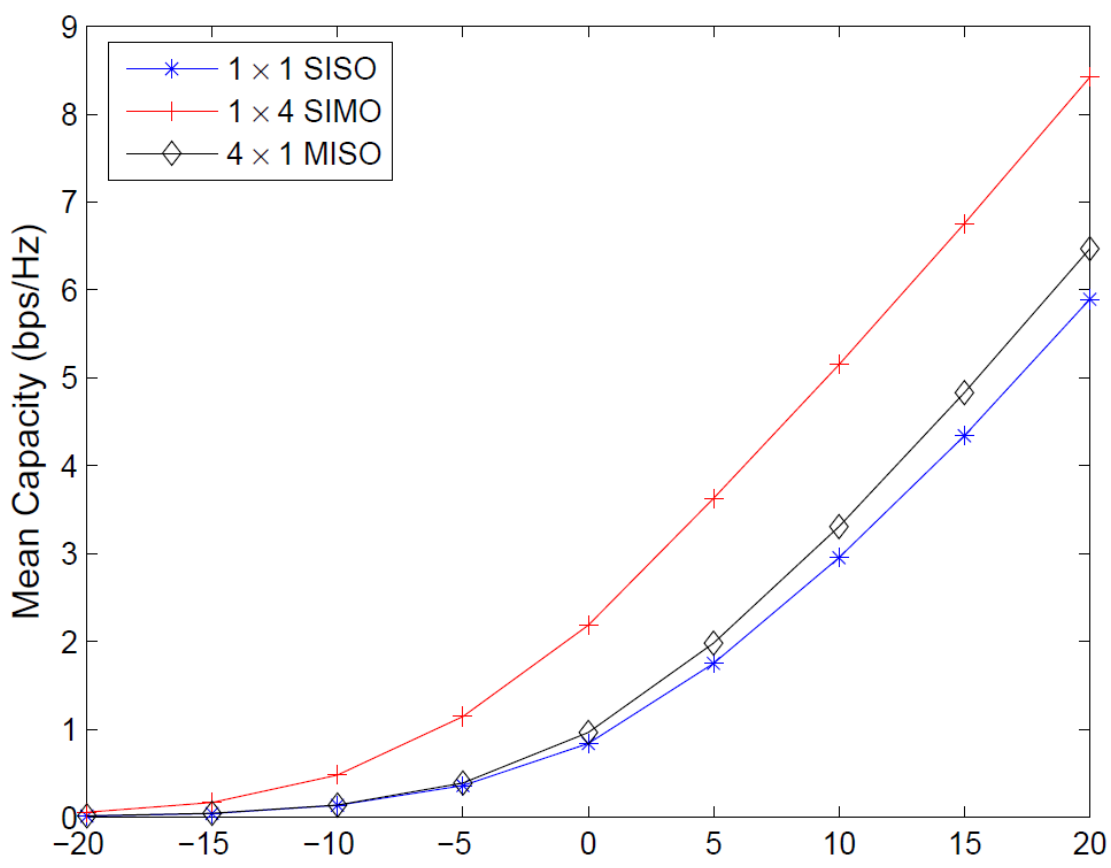

Figure 2.4: Comparison of mean capacity of SISO, SIMO and MISO systems as a function of SNR. [85]

\subsubsection{MIMO channel capacity [29]}

Capacity is a fundamental limit on the spectral efficiency that a communication channel can support reliably. In contrast to the capacity of the scalar additive white Gaussian noise (AWGN) channel that was first derived in [33], MIMO channels exhibit fading and encompass a spatial dimension. Throughout this thesis work, the MIMO channel capacity is used as a fundamental performance measure because it captures both the SNR and the 
multipath spatial characteristics. For a given channel realization, the channel capacity is given by

$$
c=\underset{\max _{r}\left(\boldsymbol{R}_{x}\right)=\sigma_{x}^{2}}{\log } \log \left(I_{N_{r}}+\frac{1}{\sigma_{n}^{2}} \boldsymbol{H R}_{\boldsymbol{x}} \boldsymbol{H}^{\boldsymbol{H}}\right) b / s / H z
$$

where $T_{r}($.$) denotes the trace of the matrix, and \boldsymbol{R}_{\boldsymbol{x}}=E\left\{x x^{H}\right\}$ is the transmitted signal covariance matrix. The channel capacity $c$ is the maximum data rate per unit bandwidth that can be transmitted with arbitrarily low probability of error. For a given bandwidth $\mathrm{W}$ the achievable data rate is $\mathrm{W} c$ $\mathrm{b} / \mathrm{s}$. Figure 2.5 illustrates increasing the MIMO capacity with increasing number of antennas

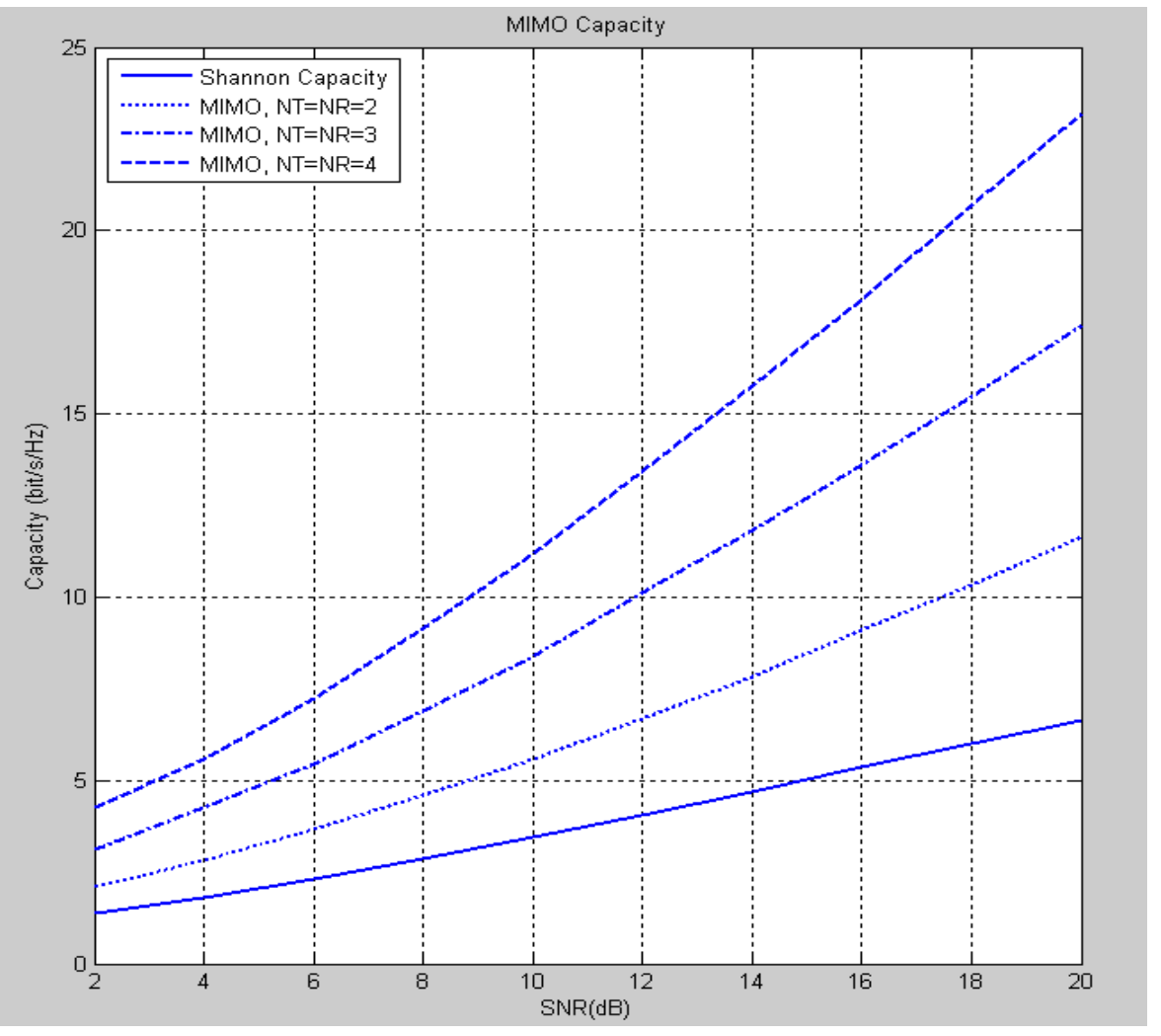

Figure 2.5: Increasing the MIMO capacity with increasing number of antennas 


\section{Chapter 3}

\section{MIMO CHANNEL MODEL CLASSIFICATION AND MULTICARRIER DELAY DIVERSITY}

The view of this chapter is to make the basic understanding of MIMO systems, space time coding and the application of MDDM in MIMO systems. The fundamentals of OFDM and its implementations with the discrete Fourier transform (DFT) are discussed. Then, the implementation of cyclic delay diversity with OFDM within the context of MIMO systems is presented. Finally, the multipath flat fading channel and the maximum ratio combining receiver are discussed.

\subsection{MIMO channel model classification}

In order to facilitate the concept of multiple-input multiple-output systems, single-input single-output (SISO) systems, single-input multiple-output (SIMO) systems and multiple-input single-output (MISO) systems models are discussed briefly. In this thesis, all signals and models are represented in complex baseband equivalent form to facilitate analysis.

\subsubsection{Single-Input Single-Output System}

The SISO system model is shown in Figure 3.1. The signal transmitted from the antenna is denoted as $x(t)$. The signal received at the receiving end, $r(t)$, passes through the channel with impulse response $h(t)$ in an additive white Gaussian noise (AWGN) environment. It is assumed that the bandwidth of the signal is small enough such that the frequency response of the channel is flat and the channel response can be given as

$$
h=|h| e^{i \varphi}
$$

where $i=\sqrt{-1}$. The relationship between the transmitted signal and receive signal is given by

$$
r(t)=\int_{-\infty}^{\infty} x(\tau) h(t-\tau) d \tau+n(t)
$$




$$
\begin{gathered}
=\int_{-\infty}^{\infty} x(\tau)|h| e^{i \varphi} \delta(t-\tau) d \tau+n(t) \\
=x(t) h+n(t)
\end{gathered}
$$

where $\delta(t)$ is the Dirac delta function and $n(t)$ is the AWGN. The received signal is the transmitted signal convolved with the channel impulse response plus added noise. [40]

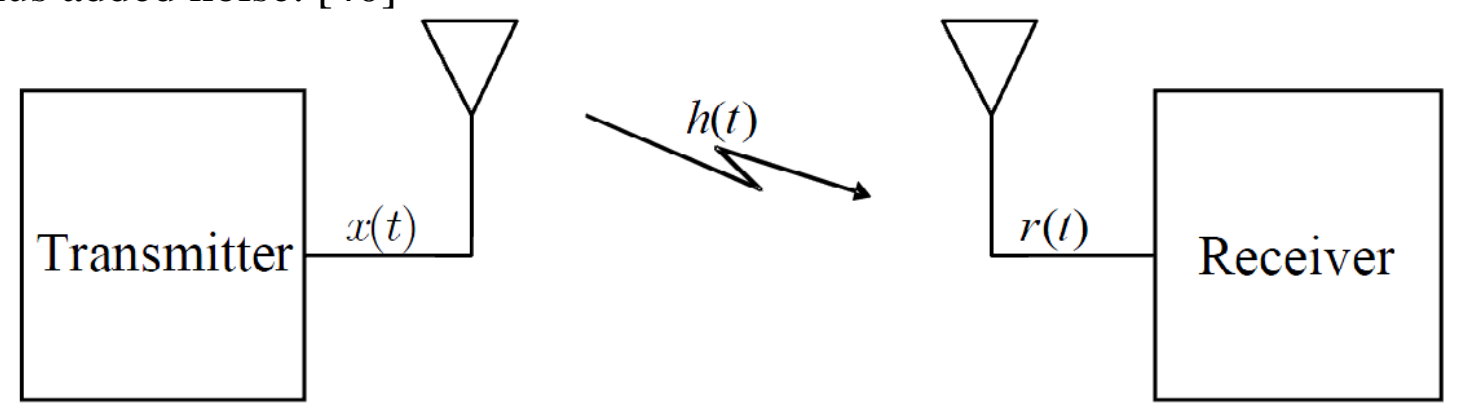

Figure 3.1: SISO system

\subsubsection{Single-Input Multiple-Output System}

A single-input multiple-output (SIMO) system with one transmit antenna and multiple $(J)$ receive antennas is illustrated in Figure 3.2.

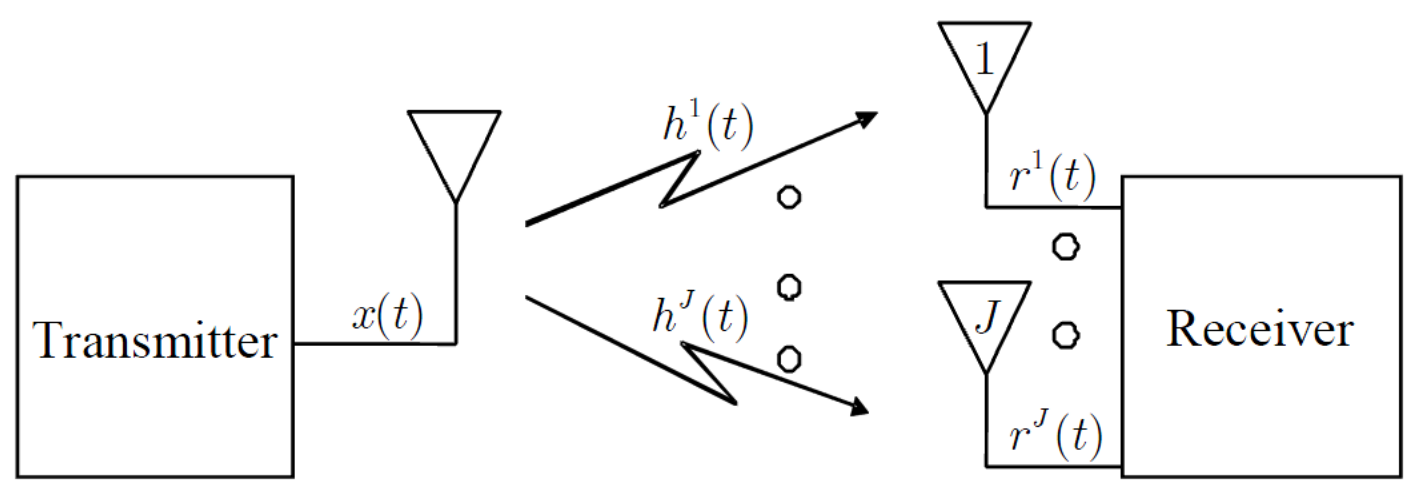

Figure 3.2: SIMO system

The received signals at the receiver end can be represented as the set of linear equations

$$
\begin{gathered}
r^{1}(t)=h^{1}(t) x(t)+n^{1}(t) \\
r^{2}(t)=h^{2}(t) x(t)+n^{2}(t) \\
\vdots \\
r^{J}(t)=h^{J}(t) x(t)+n^{J}(t)
\end{gathered}
$$


where $r^{j}(t), h^{j}(t)$ and $n^{j}(t), j=1,2,3, \ldots, J$ represent the received signal, channel impulse response and noise, respectively, at the $j^{\text {th }}$ receive antenna. If $x(t), h(t)$ and $r(t)$ are sampled at the rate of one sample per symbol, then they can be represented as $x, h, r$. The received signal can also be represented in form of vectors.

$$
\begin{gathered}
\mathbf{x}=\left[\begin{array}{lll}
\mathrm{x}^{1} & \mathrm{x}^{2} & \ldots \mathrm{x}^{\mathrm{J}}
\end{array}\right]^{\mathrm{T}} \quad \text { for } \mathrm{x}^{1}=\mathrm{x}^{2}=\ldots=\mathrm{x}^{\mathrm{J}} \\
\mathbf{h}=\left[\begin{array}{lll}
\mathrm{h}^{1} & \mathrm{~h}^{2} & \ldots \mathrm{h}^{\mathrm{J}}
\end{array}\right]^{\mathrm{T}} \\
\mathbf{n}=\left[\begin{array}{llll}
\mathrm{n}^{1} & \mathrm{n}^{2} & \ldots \mathrm{n}^{\mathrm{J}}
\end{array}\right]^{\mathrm{T}}
\end{gathered}
$$

Now, the received vector is represented as

$$
\boldsymbol{r}=\boldsymbol{h} \cdot \boldsymbol{x}+\boldsymbol{n}
$$

where $\mathbf{x}, \mathbf{h}$ and $\mathbf{n}$ are transmission, channel and noise vectors, respectively and operator ' .' denotes element by element multiplication.

\subsubsection{Multiple-Input Single-Output System}

A multiple-input single-output (MISO) system with multiple $(L)$ transmit antennas and one receive antenna is illustrated in Figure 3.3. The receive antenna receives a sum of all the signals transmitted by each antenna and can be represented as

$$
r=h^{1} x^{1}+h^{2} x^{2}+\ldots+h^{L} x^{L}+n
$$

where $x^{l}$ and $h^{l}, l=1,2,3, \ldots, L$ are the transmitted signal and channel response from transmit antenna $l$ to the receive antenna and $n$ is the AWGN. Equation (3.7) can also be written as

$$
r=\sum_{l=1}^{L} h^{1} x^{1}+n
$$




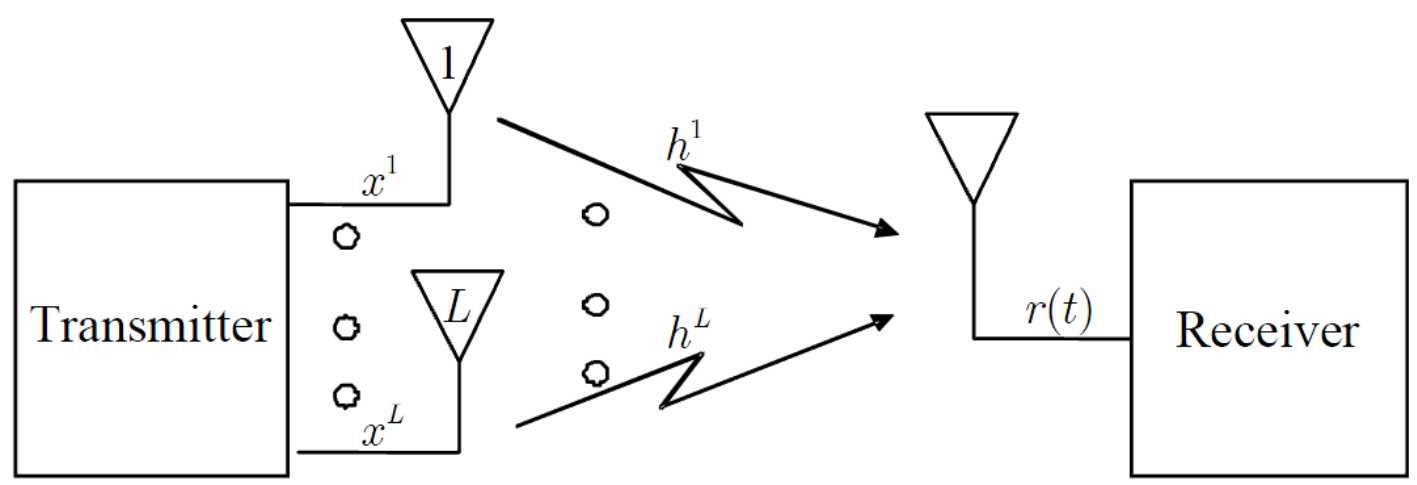

Figure 3.3: MISO system

\subsubsection{Multiple-Input Multiple-Output System}

A multiple-input multiple-output (MIMO) system with multiple $(L)$ transmit antennas and multiple $(J)$ receive antennas is illustrated in Figure 3.4. MIMO systems are the focus of this thesis. Therefore, the MIMO model is discussed in detail in this section. The representation of the model is largely based on $[34,41]$. For a faded channel, it is assumed that channel responses from each transmit antenna to each receive antenna are independent.

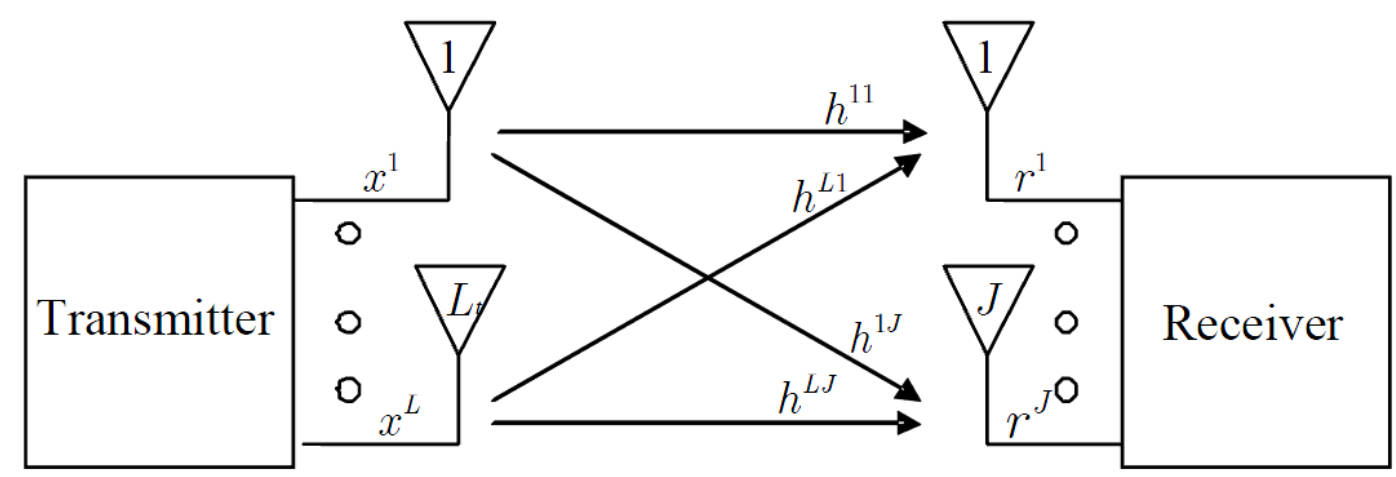

Figure3.4: MIMO system

The received signal at any receive antenna is the sum of all the signals transmitted by each transmit antenna passing through the respective channel and the AWGN.

$$
r^{j}=h^{1 j} x^{1}+h^{2 j} x^{2}+\cdots+h^{L j} x^{L}+n^{j}
$$




$$
=\left[\begin{array}{lll}
h^{1 j} & h^{2 j} & \ldots . \\
& h^{L j}
\end{array}\right]\left[\begin{array}{c}
x^{1} \\
x^{2} \\
: \\
\cdot \\
x^{L}
\end{array}\right]+n^{j}
$$

$h^{l j}$ is the channel response from transmit antenna $l$ to receive antenna $j$ where $l$ $=1,2,3, \ldots, L$ and $j=1,2,3, \ldots, J$. The system therefore can be represented in matrix form as

$$
\begin{gathered}
{\left[\begin{array}{c}
r^{1} \\
r^{2} \\
\vdots \\
\vdots \\
r^{J}
\end{array}\right]=\left[\begin{array}{cccc}
h^{11} & h^{21} & \ldots & h^{L 1} \\
h^{21} & h^{22} & \ldots & h^{L 2} \\
\vdots & \vdots & \vdots \\
\vdots & \vdots & \vdots \\
h^{J 1} & h^{J 2} & \ldots & h^{J L}
\end{array}\right]\left[\begin{array}{c}
x^{1} \\
x^{2} \\
\vdots \\
\vdots \\
x^{L}
\end{array}\right]+\left[\begin{array}{c}
n^{1} \\
n^{2} \\
\vdots \\
\vdots \\
n^{J}
\end{array}\right]} \\
\boldsymbol{r}=\boldsymbol{H} \boldsymbol{x}+\boldsymbol{n}
\end{gathered}
$$

where $\mathbf{r}$ and $\mathbf{n}$ represent the $J \times 1$ received signal and noise column vectors, $\mathbf{H}$ is a $J \times L$ complex channel matrix and $\mathbf{x}$ is the $L \times 1$ transmitted column matrix [34]. All the elements in the channel matrix are considered independent identically distributed (IID) complex Gaussian random variables (GRVs), and similarly, the elements of the noise vector are also complex Gaussian random variables. According to information theory, the optimum distributions for transmitted signals are also Gaussian. Therefore, it is considered that all elements of $\mathbf{x}$ are zero mean complex GRVs. Therefore, the covariance matrix of the transmitted signals is the same as the autocorrelation matrix and it is a diagonal matrix [34, 41]

$$
\begin{gathered}
\boldsymbol{C}_{\boldsymbol{X} \boldsymbol{X}}=\boldsymbol{R}_{\boldsymbol{X} \boldsymbol{X}} \\
\boldsymbol{R}_{\boldsymbol{X} \boldsymbol{X}}=\boldsymbol{E}\left[\boldsymbol{X} \boldsymbol{X}^{* T}\right]
\end{gathered}
$$

where $\mathbf{x}^{* T}$ denotes the transpose and component-wise complex conjugate of the transmitted matrix $\mathbf{x}$ [34]. The total transmitted power, $P$, is the sum of all the diagonal elements of the autocorrelation matrix. To facilitate the analysis for the MIMO system, assume that all the transmit antennas transmit equal power [41]. 


$$
\begin{gathered}
P=E\left[\sum_{l=1}^{L}\left|x^{l}\right|^{2}\right]=\sum_{l=1}^{L} E\left[\left|x^{l}\right|^{2}\right]=\operatorname{tr}\left(\boldsymbol{R}_{X X}\right) \\
P^{l}=E\left[\left|x^{l}\right|^{2}\right]=\frac{P}{L}
\end{gathered}
$$

where $P^{l}$ is the average power transmitted from antenna $l$. An AWGN channel is considered, and according to information theory, the optimum distribution for the transmitted signal is also Gaussian [34]. Therefore, the elements of $\mathbf{x}$ as stated in [34] are also considered independent and identically distributed (IID) Gaussian variables with zero mean. Then, the autocorrelation matrix can be written as

$$
\boldsymbol{R}_{\boldsymbol{X} \boldsymbol{X}}=\frac{P}{L} I_{L}
$$

where $I_{L}$ is the identity matrix of size $L \times L$

It is further assumed that the channel matrix is fixed at least for the duration of one symbol period and there is no attenuation due to path loss and no amplification due to antenna gain. In other words, each receive antenna receives the total transmitted power regardless of its branch. Thus, the normalization constraint for the elements of channel matrix $\mathbf{H}$ for fixed coefficient can be represented as [34]

$$
\sum_{l=1}^{L}\left|h^{j l}\right|^{2}=L \quad \text { for } j=1,2, \ldots \ldots J
$$

For a faded channel, the channel matrix elements are random variables and the normalization constraint will apply to the expected value of Equation (3.16). This normalization constraint is required for a fair comparison with SISO systems with equal power transmitted [41]. It is also assumed that the channel impulse response at that time, referred to as the channel state information (CSI), is perfectly known at the receiver by sending training symbols. [34]

The elements of the noise vector $\mathbf{n}$ are considered IID complex Gaussian random variables with zero mean and the variance of $\sigma_{o}{ }^{2}$ for both real and imaginary parts. Independence of the noise elements and zero mean imply that the autocorrelation and covariance matrix are the same diagonal matrix. Therefore, the covariance matrix $\mathbf{C}_{\mathbf{n n}}$ of the noise vector can be represented as $[34,41]$ 


$$
\begin{gathered}
\boldsymbol{C}_{\boldsymbol{n} \boldsymbol{n}}=\boldsymbol{R}_{\boldsymbol{n} \boldsymbol{n}}=E\left[\boldsymbol{n n}^{* T}\right] \\
\boldsymbol{R}_{\boldsymbol{n} \boldsymbol{n}}=\sigma_{o}^{2} \boldsymbol{I}_{L}
\end{gathered}
$$

The average signal power at receive antenna $j$ with assumed fixed channel coefficients can be given by $[34,41]$

$$
\begin{gathered}
P_{r}^{j}=E\left[r^{j} r^{j *}\right] \\
=E\left[\sum_{l=1}^{L} h^{l j} x^{l} \sum_{m=1}^{L} h^{m j *} x^{m *}\right] \\
=E\left[\sum_{l=1}^{L} \sum_{m=1}^{L} h^{l j} x^{l} h^{m j *} x^{m *}\right] \\
=\sum_{l=1}^{L} \sum_{m=1}^{L} h^{l j} h^{l j *} E\left[x^{j} x^{m *}\right] \\
=\sum_{l=1}^{L}\left|h^{l j}\right|^{2} E\left[\left|x^{l}\right|^{2}\right]
\end{gathered}
$$

where ( )* denotes complex conjugate. Substitution of Equations (3.15) and (3.16) into Equation (3.19) yields

$$
P_{r}^{j}=\sum_{l=1}^{L}\left|h^{l j}\right|^{2} E\left[\left|x^{l}\right|^{2}\right]=L \frac{P}{L}=P
$$

Then, the average signal-to-noise ratio (SNR) at each receive antenna, represented by , is given by [34, 41]

$$
\gamma=\frac{P}{\sigma_{o}^{2}}
$$

Similarly, the autocorrelation matrix for the received signal can be represented as [41]

$$
\begin{gathered}
\boldsymbol{R}_{\boldsymbol{r} \boldsymbol{r}}=E\left[\boldsymbol{r} \boldsymbol{r}^{* T}\right] \\
\boldsymbol{R}_{\boldsymbol{r} \boldsymbol{r}}=E\left[(\boldsymbol{H} \boldsymbol{x}+\boldsymbol{n})(\boldsymbol{H} \boldsymbol{x}+\boldsymbol{n})^{* T}\right]
\end{gathered}
$$




$$
\begin{gathered}
=E\left[(\boldsymbol{H} \boldsymbol{x}+\boldsymbol{n})\left((\boldsymbol{H} \boldsymbol{x})^{* T}+\boldsymbol{n}^{* T}\right)\right] \\
=E\left[\boldsymbol{H} \boldsymbol{x}(\boldsymbol{H} \boldsymbol{x})^{* T}+\boldsymbol{H} \boldsymbol{x} \boldsymbol{n}^{* T}+\boldsymbol{n}(\boldsymbol{H} \boldsymbol{x})^{* T}+\boldsymbol{n} \boldsymbol{n}^{* \boldsymbol{T}}\right] \\
=E\left[\boldsymbol{H} \boldsymbol{x}(\boldsymbol{H} \boldsymbol{x})^{* T}\right]+E\left[\boldsymbol{H} \boldsymbol{x} \boldsymbol{n}^{* T}\right]+E\left[(\boldsymbol{H} \boldsymbol{x})^{* T} n\right]+E\left[\boldsymbol{n} \boldsymbol{n}^{* \boldsymbol{T}}\right]
\end{gathered}
$$

By using the identity of transposition of a product of matrices [42] as follows

$$
(\boldsymbol{H} \boldsymbol{x})^{* T}=\boldsymbol{x}^{* T} \boldsymbol{H}^{* T}
$$

Equation (3.23) can be written as

$$
\boldsymbol{R}_{\boldsymbol{r r}}=E\left[\boldsymbol{H} \boldsymbol{x} \boldsymbol{x}^{* T} \boldsymbol{H}^{* T}\right]+E\left[\boldsymbol{H} \boldsymbol{x} \boldsymbol{n}^{* T}\right]+E\left[\boldsymbol{H}^{* T} \boldsymbol{n}^{* T} \boldsymbol{n}+E\left[\boldsymbol{n} \boldsymbol{n}^{* \boldsymbol{T}}\right]\right]
$$

If it is assumed that the channel coefficients are deterministic and signal matrix $\mathbf{x}$ and noise matrix $\mathbf{n}$ are independent with zero mean, Equation (3.25) yields

$$
\begin{gathered}
\boldsymbol{R}_{\boldsymbol{r r}}=\boldsymbol{H} \boldsymbol{E}\left[\boldsymbol{x} \boldsymbol{x}^{* T}\right] \boldsymbol{H}^{* T}+E\left[\boldsymbol{n} \boldsymbol{n}^{* T}\right] \\
=\boldsymbol{H R}_{\boldsymbol{x} \boldsymbol{x}} \boldsymbol{H}^{* T}+\boldsymbol{R}_{\boldsymbol{n} \boldsymbol{n}}
\end{gathered}
$$

\subsection{Space time coding}

Space time coding is a technique to achieve higher diversity at the receiver end to mitigate multipath fading without increasing the transmitted power or bandwidth. Space time coding holds the promise to maximize the system capacity. The system capacity is defined in [34], "The maximum possible transmission rate such that the probability of error is arbitrarily small." The capacity of SISO system is given by Shannon's capacity equation [43]

$$
\begin{gathered}
C=W \log _{2}(1+S N R) \\
C=W \log _{2}\left(1+\frac{P}{\sigma^{2}}\right)
\end{gathered}
$$

where $C, W, P$ and $\sigma^{2}$ represent capacity, bandwidth, average signal power and average noise power, respectively. The capacity of a MIMO system in a flat fading channel with perfect channel state information is given by [34] 


$$
C=W \log _{2}\left(1+L J \frac{P}{\sigma^{2}}\right)
$$

Space time coding techniques designed appropriately with MIMO systems have the potential to achieve the channel capacity in Equation (3.28). Space time coding provides the diversity both in time and space to achieve the higher performance at reduced transmitted power and without bandwidth expansion. Space time coding techniques can be classified into two main categories. The first category provides power efficiency without compromising the performance such as delay diversity, space-time block codes (STBC) and space-time turbo trellis codes (STTC). The other category, such as Bell Labs layered space-time technology (BLAST), increases the data rates with the use of bandwidth efficient modulation schemes [35].

The performance of a MIMO system can be further improved by applying forward error correction coding (FEC) with optimum interleaving at the cost of reduced data rate or increased bandwidth. FEC coding gain can be achieved without sacrificing the data rate or bandwidth by designing space time coding technique with higher rate modulation schemes. [34] This thesis is focused on achieving multicarrier delay diversity gain. The incorporation of error control coding and interleaving is left for future work.

\subsection{Multicarrier delay diversity in MIMO systems}

The delay diversity technique was the first approach proposed for MIMO systems [35]. In this scheme, delayed versions of the same signal are transmitted by multiple antennas. This simple delay diversity was originally suggested for flat fading channels [44]. This scheme has the inherent problem of increasing frequency selectivity caused by the delay diversity. Full diversity cannot be achieved without equalization [45] and equalization for MIMO systems is very difficult due to the large number of channels. Thus, the receiver design becomes much more complicated. Orthogonal frequency division multiplexing (OFDM) with delay diversity is another approach to make good use of frequency selectivity of the delay diversity. The OFDM scheme with delay diversity has a limitation as an increase in the number of transmit antennas requires an increase in the guard interval at the expense of bandwidth. If the guard interval is not as large as or larger than the delay spread of the channels, then it will cause inter-symbol interference (ISI) [45]. The OFDM scheme can be easily implemented by using the inverse discrete Fourier transform (IDFT), which can be efficiently computed by the inverse 
fast Fourier transform (IFFT). The same information can be translated back by the DFT operation. DFT and IDFT as defined in [46] are

$$
\begin{aligned}
& \operatorname{DFT}\{x[m]\}=X[k]=\sum_{n=0}^{N-1} x[m] e^{-i 2 \pi k n / N} \quad \text { for } k=0,1,2 \ldots \ldots N-1 \\
& \operatorname{DFT}\{X[k]\}=x[m]=\sum_{k=0}^{N-1} X[k] e^{-i 2 \pi k n / N} \quad \text { for } m=0,1,2 \ldots \ldots N-1
\end{aligned}
$$

Discrete Fourier transforms have a property that any circular shift in the time domain results in a phase shift in the frequency domain [46]

$$
\operatorname{DFT}\left\{x\left[(m-D)_{n}\right]\right\}=e^{-i 2 \pi k n / N} X[k] \quad \text { for } k=0,1,2 \ldots . . N-1
$$

where $\mathrm{D}$ denotes the delay in the time index and $(n)_{N}$ denotes $n$ modulo $N$. This cyclic delay property of the Fourier transforms can be used in an OFDM system design to induce diversity that can be exploited at the receiver end. To overcome the problems of a simple time delay diversity scheme in a frequency selective fading channel for MIMO-OFDM systems, a new approach of cyclic delay diversity was suggested in [39]. This simple scheme does not require any additional guard interval with an increasing number of transmit antennas. The combination of cyclic delay diversity and OFDM in MIMO systems has been referred to as multicarrier delay diversity modulation (MDDM) in this thesis and this can be considered a special type of space-time coding. For a frequency selective channel, a cyclic guard interval (cyclic prefix) of duration $G$ is added at the beginning of each OFDM symbol. This guard interval is greater than or equal to the maximum channel delay $M$ to mitigate the intersymbol interference (ISI). The orthogonality of subcarriers is paramount for OFDM. The cyclic prefix converts a linear convolution channel into a circular convolution channel and the interference from the previous symbols will only affect the guard interval. This restores the orthogonality at the receiver. Adding zeros as the guard interval can alleviate the interference between OFDM symbols. For a flat fading channel, the guard interval can be eliminated to increase the data rate. [35] The space time code for MDDM can be represented as [35] 


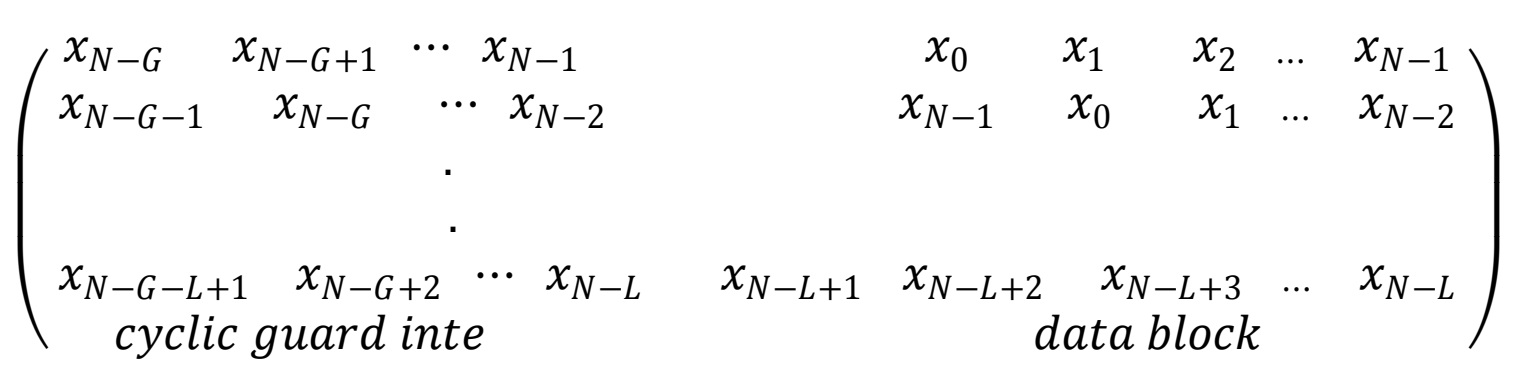

where $G$ is the length of the guard interval and $N$ is the number of points in the IDFT. The MDDM scheme has some advantages over space time block coding (STBC) and space time trellis coding (STTC). STBCs require all block codes be orthogonal and full rate transmission is not achievable for more than two transmit antennas. The complexity of STTC increases exponentially with the number of transmit antennas. STBCs and STTCs are fixed for a combination of transmit and receive antennas and they do not offer any flexibility in changing the number of antennas at either end. For each combination of the number of antennas, a new space-time code is required. These codes were designed for flat fading channels. For frequency selective channels, they cannot be used without equalization and other compensation techniques. However, the MDDM space time code can be used with any combination of transmit and receive antennas with little modification in the receiver design. The MDDM is based upon the OFDM modulation scheme. Therefore, orthogonal frequency division multiplexing fundamentals are discussed very briefly in the following sections. [34, 35]

\subsection{Orthogonal frequency division multiplexing}

Broadband MIMO wireless communication systems operating in a rich scattering environment usually face unacceptable inter-symbol interference (ISI) from multipath propagation and their inherent delay spread. Thus, the channel exhibits frequency selective fading. Orthogonal Frequency Division Multiplexing (OFDM) is a powerful, multicarrier technique for combating ISI. In effect, it transforms a frequency selective channel into a set of parallel flat fading channels and hence, the signals on each subcarrier undergo narrowband fading. In order to deal with frequency selective nature of broadband wireless channels, MIMO can be combined with OFDM. Thus, the combination of the two technologies can achieve higher data rates at high quality of service [36-39]. OFDM is a multi-carrier technique that operates with specific orthogonality constraints between the sub-carriers, which enables it to achieve a very high spectral efficiency. It can be implemented 
using IDFT (inverse discrete Fourier transform) at the transmitter and DFT (discrete Fourier transform) at the receiver which are in turn implemented using IFFT (inverse fast Fourier transform) and FFT (fast Fourier transform) algorithms respectively when the number of subcarriers are powers of two. The data symbols to be transmitted are first converted serial to parallel and each $\mathrm{N}_{\mathrm{c}}\left(\mathrm{N}_{\mathrm{c}}\right.$ is the number of OFDM subcarriers) of these symbols are OFDM modulated using IFFT at the transmitter (assuming all subcarriers are utilized) as shown in the block diagram of Figure 3.5. The output of IFFT is $\mathrm{N}_{\mathrm{c}}$ samples of a time domain OFDM symbols. OFDM combats ISI by using guard interval, the duration of time in which the multipath components are allowed to die out. To reduce the receiver complexity, a guard symbol which is a replica of the last $\mathrm{N}_{\mathrm{g}}$ samples (called cyclic prefix) of the OFDM symbol, is introduced during the guard interval. This converts a linear convolution of a signal and the channel to a circular convolution and thereby causing the FFT of the circularly convolved signal and channel to simply be the product of their respective FFTs. The guard length $\mathrm{N}_{\mathrm{g}}$ is chosen to be greater than the maximum channel delay spread. Thus, the relative length of the cyclic prefix depends on the ratio of the channel delay spread to the OFDM symbol duration. In the block diagram of MIMO-OFDM systems shown in Figure 3.5, for simplicity, channel coding and interleaving of the source bit streams are not included. On the transmitter side the source data bits are mapped to a constellation by digital modulator and then encoded (de-multiplexed) by MIMO encoder (de multiplexer) on to different transmission layers. Just before transmission the symbols of each transmission layer are OFDM modulated where data symbols are serial to parallel converted, passed through IFFT filters and then in order to avoid ISI cyclic prefix (CP) is added to each OFDM symbols [39, 46]. At the receiver side, the reverse process of transmitter takes place. The received samples are OFDM demodulated where, first, $\mathrm{CP}$ is removed, then the remaining samples are serial to parallel converted and finally passed through FFT filters. The outputs of the FFT filters are fed to the MIMO decoders where signal detection takes place per sub-carrier [46]. 


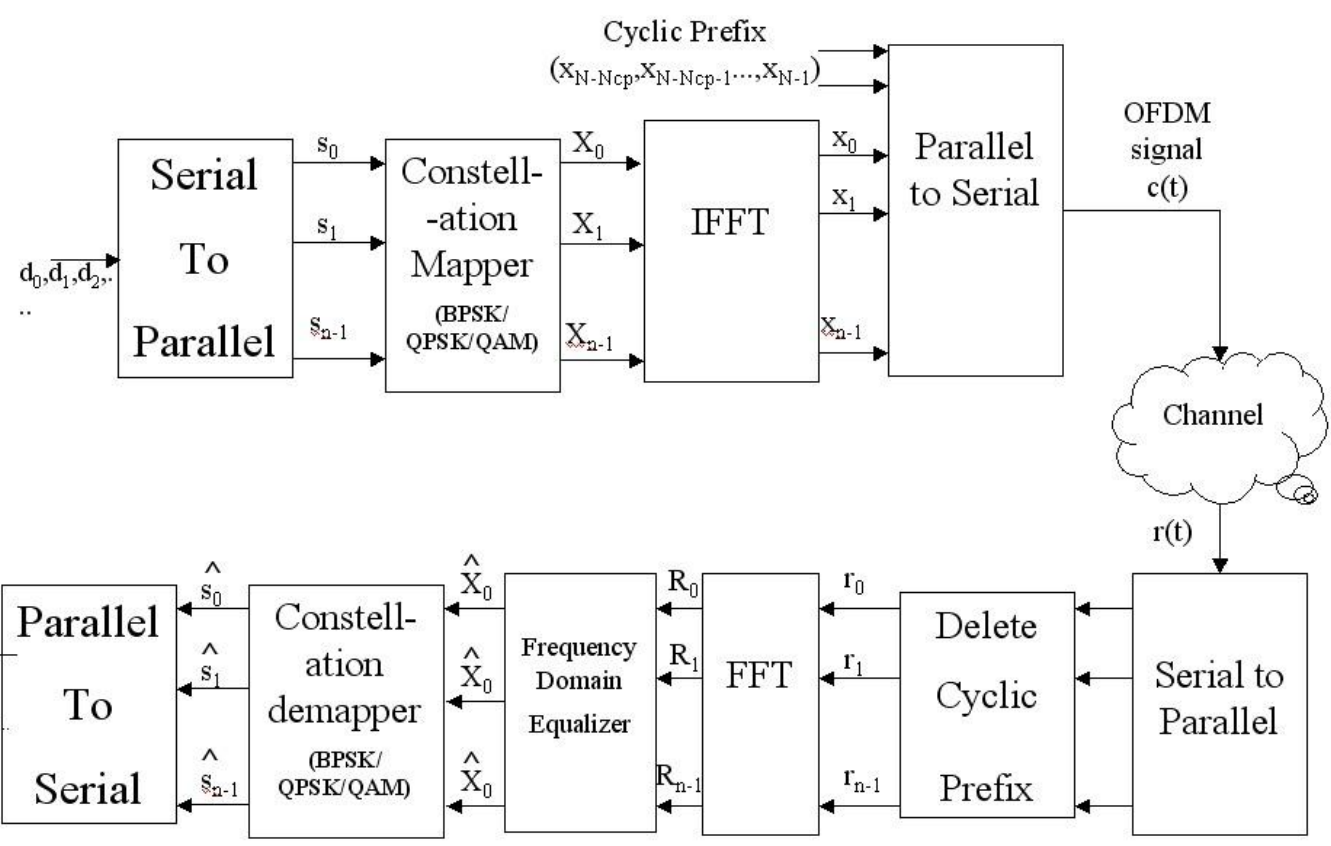

Figure 3.5: Block diagram of OFDM modulation using IFFT

In conventional sequential data transmission over a single carrier, the data rate has a limit imposed by the availability of frequency spectrum and the delay spread of the channel. The bandwidth required is inversely proportional to the data symbol duration, which means that the highest achievable symbol rate is limited by the available bandwidth [49]. Considering a fixed delay spread of the channel, the ISI also increases with the increase in the symbol rate as delayed copies of the symbols coming from the multipath can have significant overlap with the original symbol. The lessening of ISI will require equalization which further adds to the complexity of the system [50]. These problems can be mitigated by transmitting the data in parallel on multiple carriers with a reduced data rate on each carrier compared to the overall data rate. The reduced data rate will require reduced bandwidth which should not be more than the coherence bandwidth of the channel to avoid frequency selective fading. This multicarrier system can be designed by classical frequency division multiplexing (FDM) [34]. In this scheme, the carriers need to be well apart in frequency domain to avoid inter-carrier interference. Therefore, a guard frequency band is required between two consecutive subcarriers which makes this scheme highly inefficient in frequency spectrum utilization. This problem can be eliminated by using minimum-spaced orthogonal carriers. In OFDM, all carriers are allowed to overlap by maintaining orthogonality of all the subcarriers, which increases bandwidth 
efficiency [47]. The comparison of bandwidth efficiency for FDM and OFDM is illustrated in Figure 3.6.
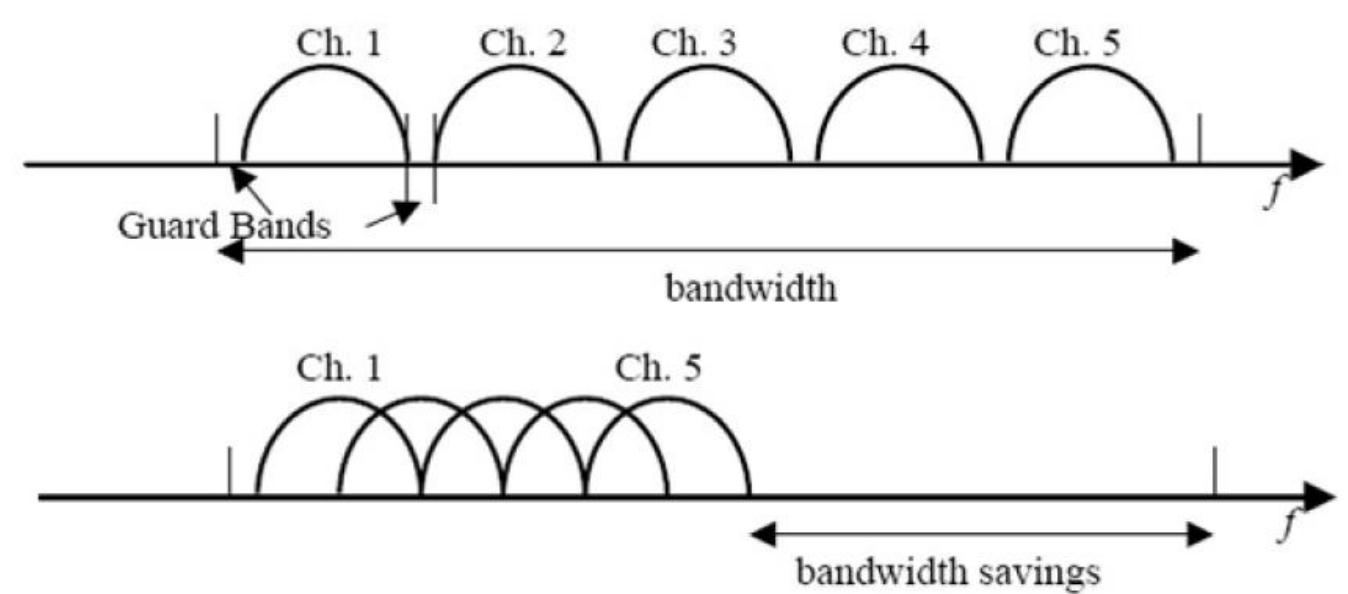

Figure 3.6: Comparison of bandwidth efficiency between FDM and OFDM

In OFDM, to maintain the orthogonality of the subcarrier channels, the correlation between signals transmitted on subcarriers must be zero. Assume that the available bandwidth for the OFDM system is $\Delta W$ and it is to be divided in $K$ subcarriers. The input serial data stream is to be converted into $K$ parallel data streams which are assigned to the $K$ subcarriers [34]. The symbol duration of the input serial data is ' $T_{s}^{\prime}$ with serial data rate of $f_{s}^{\prime}=\frac{1}{T_{s}}$. Therefore, if the number of parallel data streams is equal to the number of OFDM subcarriers, the symbol duration for OFDM will be

$$
T_{s}=K T_{s}^{\prime}
$$

Equation (3.33) indicates that the symbol duration of an OFDM signal is $K$ times larger than that of single serial stream symbol duration. Therefore, the OFDM scheme has the inherited advantage over single carrier modulation techniques to mitigate ISI and frequency selectivity of the channel. The OFDM transmitted signal $S(t)$ can be written as

$$
S(t)=\sum_{m=0}^{K=1} A\left\{d_{I_{m}} \cos \left(2 \pi f_{m} t\right)-d_{Q_{m}} \sin \left(2 \pi f_{m} t\right)\right\}
$$




$$
=\sum_{m=0}^{K-1} a_{m}\left\{\frac{d_{I_{m}}}{\sqrt{d_{I_{m}}^{2}+d_{Q_{m}}^{2}}} \cos \left(2 \pi f_{m} t\right)-\frac{d_{Q_{m}}}{\sqrt{d_{I_{m}}^{2}+d_{Q_{m}}^{2}}} \sin \left(2 \pi f_{m} t\right)\right\}
$$

where $A$ is a constant, $d_{I_{m}}, d_{Q_{m}}$ are the information-bearing components of the signal and

$$
a_{m}=A \sqrt{d_{I_{m}}^{2}+d_{Q_{m}}^{2}}
$$

Using the trigonometric identity

$$
\cos (\alpha+\beta)=\cos (\alpha) \cos (\beta)-\sin (\alpha) \sin (\beta)
$$

$S(t)$ can be written as

$$
\begin{gathered}
\qquad(t)=\sum_{m=0}^{K-1} a_{m \cos \left(2 \pi f_{m} t+\theta_{m}\right)} \\
=\sum_{m=0}^{K-1} a_{m}\left\{\cos \left(2 \pi f_{m} t\right) \cos \left(\theta_{m}\right)-\sin \left(2 \pi f_{m} t\right) \sin \left(\theta_{m}\right)\right\} \\
\text { Where } \theta_{m}=\tan ^{-1}\left(d_{Q_{m}} / d_{I_{m}}\right) \text {. The correlation between any two symbols }
\end{gathered}
$$
transmitted on separate subcarriers, represented as $R_{i j}$ must be equal to zero to maintain the orthogonality of subcarriers [34].

$$
\begin{gathered}
R_{i j}=\int_{-\infty}^{+\infty} s_{i}(t) s_{j}(t) d t \\
=\int_{0}^{T_{s}} a_{i} \cos \left(2 \pi f_{i} t+\theta_{i} t\right) a_{j} \cos \left(2 \pi f_{j} t+\theta_{j} t\right) d t
\end{gathered}
$$

Using the trigonometric identity

$$
\cos (\alpha) \cos (\beta)=\frac{1}{2} \cos (\alpha+\beta)+\frac{1}{2} \cos (\alpha-\beta)
$$


Equation (3.37) can be written as

$$
\begin{gathered}
R_{i j}=\frac{a_{i} a_{j}}{2} \int_{0}^{T_{S}}\left(\cos \left(2 \pi\left(f_{i}+f_{j}\right) t+\left(\theta_{i}+\theta_{j}\right) t\right)+\cos \left(2 \pi\left(f_{i}-f_{j}\right) t+\right.\right. \\
\left.\left.\left(\theta_{i}-\theta_{j}\right) t\right)+\right) d t
\end{gathered}
$$

where $a_{i}, a_{j}$ are constant for the symbol duration.

For $2 \pi\left(f_{i}+f_{j}\right) \gg \frac{1}{T_{s}}$ Equation (3.39) can be written as

$$
R_{i j}=\frac{a_{i} a_{j}}{2} \int_{0}^{T_{s}} \cos \left(2 \pi\left(f_{i}-f_{j}\right) t+\left(\theta_{i}-\theta_{j}\right) t\right) d t
$$

From Equation (3.40), $R_{i j}=0$ if

$$
\left(f_{i}-f_{j}\right) T_{s}=M \text { where } \mathrm{M} \in \text { set of positive integers }
$$

$$
\Rightarrow\left(f_{i}-f_{j}\right)=\frac{M}{T_{S}}
$$

Therefore, minimum frequency separation between two consecutive subcarriers to maintain orthogonality must be

$$
\Delta f=\frac{1}{T_{s}}=f_{s}
$$

Where $f_{s}$ is the rate of OFDM symbols.

\subsubsection{Generation of OFDM}

From the earlier discussion of orthogonality in an OFDM signal, all subcarriers are orthogonal to one another and the center frequencies of any two subcarriers differ by a positive integer multiple of $\Delta f$. In spite of the overlapping of subcarrier frequency bands, the transmitted symbol can be recovered at the receiver end without any interference due to zero correlation of these subcarriers. In OFDM, serial data symbols are converted to parallel to form one OFDM symbol to be assigned to $K$ subcarriers. Now, the bandpass signal can be represented as

$$
S(t)=\sum_{m=0}^{K-1} A\left\{d_{I_{m}} \cos \left(\omega_{m} t\right)-d_{Q_{m}} \sin \left(\omega_{m} t\right)\right\}
$$


where the subcarrier frequencies $\omega_{m}=2 \pi f_{m}$ and $f_{m}=f_{o}+m \Delta f$.The equation (3.43) can be written as

$$
\begin{array}{r}
S(t)=\operatorname{Re}\left\{\sum_{m=0}^{K-1} A\left(d_{I_{m}}+i d_{Q_{m}}\right) e^{i \omega_{m} t}\right\} \\
=\operatorname{Re}\left\{\sum_{m=0}^{K-1} d[m] e^{i \omega_{m} t}\right\} \\
=\operatorname{Re}\left\{\sum_{m=0}^{K-1} d[m] e^{i\left(2 \pi\left(f_{o}+m \Delta f\right) t\right)}\right\}
\end{array}
$$

Where $d[m]=A d_{I_{m}}+i A d_{Q_{m}}$ and the complex envelope of the transmitted signal is denoted by

$$
\bar{D}(t)=\sum_{m=0}^{K-1} d[m] e^{i 2 \pi m \Delta f t}
$$

Matched filters or correlation demodulators can be used at the receiver end to recover the symbol for each subcarrier. Subsequently, for the implementation of this scheme, $K$ modulators and $K$ matched filters are required. As the number of subcarriers increases, the complexity of the system also increases. For a large number of subcarriers, the complexity of the system makes it impractical. [34]

For making this scheme more practical, consider sampling the complex envelope signal $\widetilde{D}$ at the rate of $K_{s} / T$, i.e., the sampling interval is $T_{s} / K$. Now using Equation (3.42), Equation (3.45) can be represented as

$$
\begin{gathered}
\widetilde{D}\left[\frac{M T_{s}}{K}\right]=\sum_{m=0}^{K-1} d[m] e^{i 2 \pi m \Delta f M T_{s} / K} \\
=\sum_{m=0}^{K-1} d[m] e^{i 2 \pi m \Delta f M T_{s} / T_{S} K} \\
=\sum_{m=0}^{K-1} d[m] e^{i 2 \pi m \Delta f M / K}
\end{gathered}
$$


Comparison of Equations (3.30) and (3.46) clearly yields that the right side of Equation (3.46) is the IDFT of $d[m]$ scaled by $K$.

$$
\widetilde{D}=\operatorname{KIDFT}\{d[m]\}
$$

Equation (3.47) facilitates the implementation of an OFDM system by taking the IDFT of the original data stream. Therefore, implementation of the OFDM scheme is completely digital. The complexity of the system is decreased as compared to the multioscillator based OFDM modulation technique. Even for large $K$, IDFT/DFT can be efficiently implemented by using fast Fourier transforms (FFT) which make computation much faster [34]. Similarly, the receiver performs the FFT operation on the received signal to recover the original parallel data. Employing the FFT in the receiver drastically reduces the complexity as compared to employing coherent demodulators. The receiver can separate the subcarriers simply by using baseband signal processing techniques. These subcarrier signals can be integrated for the duration of the symbol period to produce decision variables for the estimation of the transmitted data.

\subsubsection{Cyclic Guard Interval}

As previously discussed, the OFDM modulation technique has the advantage of reducing the ISI caused by a multipath channel. This is achieved by converting serial data at a high rate into parallel streams each at a lower data rate and increasing the symbol transmission duration on orthogonal carriers. In order to eliminate the ISI completely due to the time delay spread of the multipath channel, a guard interval is added before each OFDM symbol. The length of the guard interval is to be greater than the anticipated delay spread of the channel [34]. This guard interval insertion costs both in terms of power and data rate or bandwidth. If the length of the guard interval is less than the delay spread of the channel, then multiple delayed versions will induce ISI and inter-carrier interference by affecting the orthogonality of the subcarriers due to overlapping of different OFDM symbols [47]. Taking advantage of the cyclic nature of the DFT represented in Equation (3.31), the guard interval can be constructed by some cyclically shifted portion of the OFDM symbol. The addition of this cyclic guard interval has already been depicted in the space time code equation (3.32). Additionally, this cyclic prefix also facilitates the synchronization of the carrier frequency and timing to obtain the orthogonality of the subcarriers [45]. The addition of the cyclic guard interval is illustrated in Figure 3.7. 


\begin{tabular}{|c|c|c|c|c|c|c|}
\hline GI & OFDM Symbol & GI & OFDM Symbol & GI & OFDM Symbol & GI \\
3 & 2 & 2 & 1 & 1 & 0 & 0 \\
\hline
\end{tabular}

Figure 3.7: The addition of the cyclic guard interval

Effect of Cyclic Prefix on Es/N0: The following diagram illustrates in figure 3.8 the concept of cyclic prefix. Each OFDM symbol contains both useful data and overhead (in the form of cyclic prefix). The bit energy represents the energy contained in the useful bits. In this case, the bit energy is spread over $\mathrm{N}$ bits (where $\mathrm{N}$ is the FFT size). On top of the useful data, additional $\mathrm{N}_{\mathrm{cp}}$ bits are added as cyclic prefix, which forms the overhead. So if the entire OFDM symbol is considered, the symbol energy is spread across $\mathrm{N}+\mathrm{Ncp}$ bits.

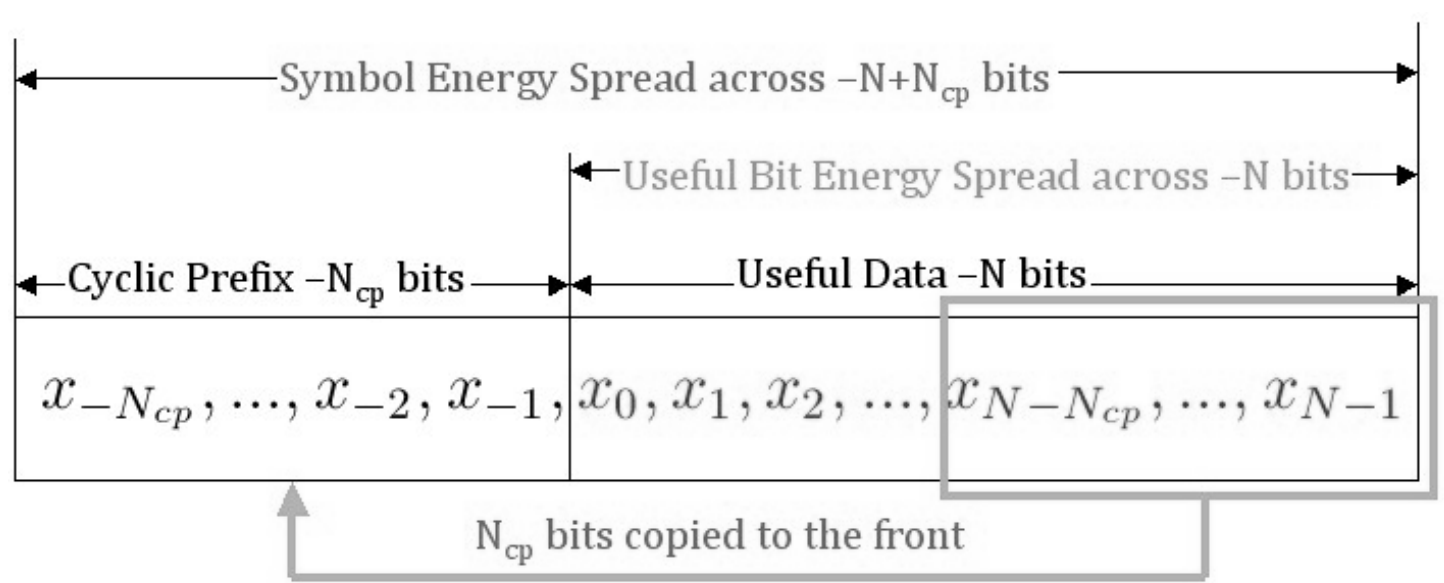

Figure 3.8: Diagram of the concept of cyclic prefix [Wikipedia]

This relationship is given as

$$
E_{s}\left(N+N_{c p}\right)=N E_{b}
$$

which translates to,

$$
E_{s}=\frac{N}{N+N_{c p}} E_{b}
$$

\subsection{The multipath and fading channel}

In terrestrial wireless communications, the line of sight (LOS) path for signal reception is often not available. The signal arriving at the receive antenna is comprised of many replicas of the signal coming from a number of reflectors and scatters present in the medium of transmission. This phenomenon is 
known as multipath. Even when the LOS path signal is present, the multipath components are usually also present. [48, 49] In this section, a general view of the multipath channel is presented to understand its nature and a baseband equivalent model is derived for use in simulation. The MDDM technique will be tested in a multipath faded channel for performance analysis and evaluation.

In a multipath channel, the signal travels via several different paths before arriving at the receive antenna. Due to the different path lengths involved, the amplitude and phase of these received replicas are not the same. For example, if a short duration pulse is transmitted in a multipath channel, then a train of pulses of different amplitudes, phases and arrival times, will be received. As a result, the received signal can significantly vary in amplitude and phase and the spectral components of the signal are affected differently by the multipath faded channel. Therefore, the frequency response of the channel may not be flat over the entire bandwidth of the signal. Multipath components of the signal create time-spreading of the signal and cause inter-symbol-interference $[48,49]$. The channel conditions may not remain constant over time and the media composition (e.g., stratosphere, ionosphere) may also change. Furthermore, the number and position of the reflectors and scatters cannot be considered fixed. In mobile communications, due to the motion of transmitter and receiver, the multipath arrangements can never be assumed constant and Doppler shift in signal frequency proportional to the relative velocity is also observed. Therefore, the multipath fading channel is a time varying channel [48]. Time varying and time spreading aspects of the channel cannot be predicted or calculated precisely. For creating a good model of the channel, these parameters are measured, and based on these measured statistics, the channel can be characterized. Doppler spread in the signal frequency describes the time varying nature of the channel. The coherence time is inversely proportional to the Doppler shift. The coherence time is the statistical measure over which the channel response does not change significantly. To characterize the channel, the coherence time is compared with the symbol duration. If the coherence time is less than the symbol durations, then the channel is called a fast fading channel. In the case of coherence time greater than the symbol duration, it is described as a slow fading channel $[48,49]$. The multipath delay spread is characterized by the coherence bandwidth. Coherence bandwidth has been defined in [50] as, "a statistical measure over which the frequency response of the channel is considered flat." If the bandwidth of the signal is greater than the coherence bandwidth of the channel, then different frequency components will be 
attenuated differently and the phase variations will also be nonlinear. This type of channel is said to be a frequency selective fading channel. If the coherence bandwidth of the channel is greater than the signal bandwidth, then all the frequency components will face flat fading with almost linear phase changes. This type of channel is said to be a frequency nonselective fading channel $[48,49]$. This fading channel can be modeled statistically as there are a large number of variables affecting the channel response. Most of these variables are random in nature and several probability distributions can be considered to model these random variables [49]. When there are a large number of reflectors and scatters in the physical channel, the cumulative effect of this large number of random variables, as per the central limit theorem, leads to a Gaussian process model for the channel response. In the case of no LOS component present, the process has zero mean with magnitude following the Rayleigh probability distribution and the phase is uniformly distributed on [ $-\pi$ to ] [48, 49].

\subsubsection{Flat Rayleigh Fading Channel}

A slow fading frequency nonselective channel was simulated to test the multicarrier delay diversity modulation scheme. The channel fading gain was kept fixed for the symbol duration to make it a slow fading channel. Considering the no line of sight (LOS) path case, a flat fading channel is usually simulated using a Rayleigh distribution for the magnitude of the channel response [49]. The Rayleigh distribution is a special case of the Ricean distribution with no line of sight component. The probability density function for a Rayleigh random variable can be derived from the Ricean probability density function as defined in [49]

$$
f_{A_{c}}\left(a_{c}\right)=\frac{a_{c}}{\sigma^{2}} \exp \left(\frac{-\left(a_{c}^{2}+\alpha^{2}\right)}{2 \sigma^{2}}\right) I_{o}\left(\frac{\alpha a_{c}}{\sigma^{2}}\right) u\left(a_{c}\right)
$$

where $I_{o}($.$) is the modified Bessel function of the first kind of zero order, u($. is the unit step function and $\alpha^{2}$ is the power in the LOS signal component and the average received signal power is

$$
\begin{aligned}
& \overline{s^{2}(t)}=\overline{a_{c}^{2}}=\alpha^{2}+2 \sigma^{2} \\
& u\left(a_{c}\right)=\left\{\begin{aligned}
-1, & a_{c} \geq 0 \\
0, & a_{c}<0
\end{aligned}\right.
\end{aligned}
$$


For the Rayleigh distribution case, there is no line of sight component so $\alpha=$ 0 and $I_{o}=1$. Using Equation (3.48), the Rayleigh probability density function can be represented as

$$
f_{A_{c}}\left(a_{c}\right)=\frac{a_{c}}{\sigma^{2}} \exp \left(\frac{-a_{c}^{2}}{2 \sigma^{2}}\right) u\left(a_{c}\right)
$$

For the baseband simulation of a Rayleigh random variable, two zero mean independent real Gaussian random variables were summed as $X+j Y$ [50]. The magnitude of this complex random quantity is the desired Rayleigh random variable and simulates the magnitude of the channel frequency response. The derivation of the proof that the complex sum of two zero mean Gaussian random variables has magnitude with Rayleigh distribution and phase uniformly distributed in [- $-\pi, \Pi$ ] is largely based on [41]. Let $X$ and $Y$ be two zero mean independent identically distributed (IID) Gaussian random variables and their complex sum is represented by

$$
z=X+j Y
$$

As $Z$ is to simulate the frequency response of a Rayleigh flat fading channel, it can be written as

$$
\begin{gathered}
Z=h e^{i \theta} \\
V=\left|Z^{2}\right|=X^{2}+Y^{2}
\end{gathered}
$$

Since $X$ and $Y$ are zero mean IID Gaussian random variables (GRVs)

$$
\begin{gathered}
\bar{X}=\bar{Y}=0 \\
\sigma_{X}^{2}=\sigma_{Y}^{2}=\sigma^{2}
\end{gathered}
$$

The random variable $V$ is a sum of two squared zero mean GRVs and is called a central chi-squared random variable of degree 2 . The probability density function for a central chi-squared random variable of degree $n$ can be given as [49] 


$$
f_{v}(v)=\frac{1}{\sigma^{n} 2^{n / 2} \Gamma\left(\frac{n}{2}\right)} v^{(n / 2)-1} e^{-v / 2 \sigma^{2}} u(v)
$$

where $n$ is the number of independent variables and $\Gamma($.$) is the gamma$ function. For a central chi-squared random variable of degree two $(n=2)$, Equation (3.57) can be rewritten as

$$
f_{v}(v)=\frac{1}{2 \sigma^{2} \Gamma(1)} e^{-v / 2 \sigma^{2}} u(v)
$$

Since

$$
\Gamma(1)=(1-1) !=1
$$

[48], the probability density function of $V$ as per Equation (3.58) can be written as

$$
f_{v}(v)=\frac{1}{2 \sigma^{2}} e^{-v / 2 \sigma^{2}} u(v)
$$

The magnitude of the simulated Rayleigh flat faded channel frequency response can be written as

$$
h=|Z|=\sqrt{V}
$$

The probability density function for $h$ can be obtained by transforming the probability density function of $V$ given in Equation (3.58) according to [51]

$$
f_{H}(h)=\left.\frac{1}{|d h / d v|} f_{v}(v)\right|_{v=h^{2}}
$$

where

$$
\frac{d h}{d v}=\frac{1}{2(v)^{1 / 2}}
$$

Substituting Equations (3.60) and (3.63) into Equation (3.62) yields

$$
f_{H}(h)=2\left(h^{2}\right)^{1 / 2} \frac{1}{2 \sigma^{2}} e^{-h^{2} / 2 \sigma^{2}} u(h)
$$




$$
f_{H}(h)=\frac{h}{\sigma^{2}} e^{-h^{2} / 2 \sigma^{2}} u(h)
$$

Comparison of Equations (2.51) and (2.64) clearly indicates that the magnitude of the complex sum of two zero mean IID GRVs follows the Rayleigh distribution. The mean and variance of $h$ as given in [51] are

$$
\begin{gathered}
\bar{h}=\sigma \sqrt{\frac{\pi}{2}} \\
\sigma_{h}^{2}=\sigma^{2}\left(2-\frac{\pi}{2}\right)
\end{gathered}
$$

Now, the probability distribution function for the phase, $\theta$, of $Z$ is to be derived.

The phase $\theta$ can be represented as [41]

$$
=\left\{\begin{array}{l}
\theta \\
\tan ^{-1}\left(\frac{Y}{X}\right)-\pi \text { if }-\infty<X<0 \text { and }-\infty<Y \leq 0 \quad(\text { referred to as case } A) \\
\tan ^{-1}\left(\frac{Y}{X}\right) \text { if } 0<X<\infty \text { and }-\infty<Y<\infty \quad(\text { referred to as case } B) \\
\tan ^{-1}\left(\frac{Y}{X}\right)+\pi \text { if }-\infty<X<0 \text { and } 0 \leq Y<\infty \quad(\text { referred to as case } c)
\end{array}\right.
$$

Let the ratio of zero mean IID GRVs $X$ and $Y$ be defined as [41, 52]

$$
R=\frac{Y}{X}
$$

The joint probability density function of $X$ and $Y$ can be written as

$$
f_{X Y}(x, y)=f_{X}(x) f_{Y}(y)=f_{X}(x) f_{x}(y)
$$

where

$$
f_{X}(x)=\frac{1}{\sqrt{2 \pi \sigma^{2}}} e^{-x^{2} / 2 \sigma^{2}}
$$


For case $\mathrm{A},-\infty<X<0$ and $-\infty<Y \leq 0$ which implies $-\pi \leq \theta \leq-\pi / 2$. The conditional cumulative distribution function of $R$ can be represented as [41, $52]$

$$
\begin{gathered}
F_{R \mid A}(r)=\operatorname{Pr}\{R<r \mid A\}=\operatorname{Pr}\left\{\frac{Y}{X}<r \mid A\right\} \\
=\int_{-\infty}^{0} \int_{r x}^{0} f_{X Y \mid A}(x, y) d y d x \\
=4 \int_{-\infty}^{0} \int_{r x}^{0} f_{X Y \mid A}(x, y) d y d x
\end{gathered}
$$

where it is noted that

$$
\int_{r x}^{0} f_{X Y \mid A}=\left\{\begin{array}{lr}
4 f_{X Y(x, y)} \text { if }-\infty<x<0 \text { and }-\infty<y<0 \\
0 & \text { otherwise }
\end{array}\right.
$$

The conditional probability density function can be obtained by taking the derivative of the conditional cumulative distribution function with respect to random variable

$r$

$[41$,

$52]$

$$
f_{R \mid A}(r)=\frac{d F_{R \mid A}(r)}{d r}=4 \frac{d}{d r}\left[\int_{-\infty}^{0} \int_{r x}^{0} f_{X Y \mid A}(x, y) d y d x\right]
$$

From Leibniz's rule $[41,52]$

$$
f_{R \mid A}(r)=4 \int_{-\infty}^{0}-x f_{X Y}(x, r x) d x
$$

Substituting Equation (3.69) and (3.70) into Equation (3.74) yields [41, 52]

$$
f_{R \mid A}(r)=-\frac{1}{\pi \sigma^{2}}\left[\int_{-\infty}^{0} e^{-x^{2}\left(\frac{1+r^{2}}{2 \sigma^{2}}\right)}(2 x) d x\right]
$$


Equation (3.75) can be represented as

$$
f_{R \mid A}(r)=\frac{2}{\pi\left(1+r^{2}\right)} \quad 0 \leq r \leq \infty
$$

For case A,

$$
\begin{gathered}
\theta=\tan ^{-1}(r)-\pi \\
\left|\frac{d \theta}{d r}\right|=\frac{1}{1+r^{2}}
\end{gathered}
$$

The conditional probability density function of $\theta$ can be attained from Equation (3.76)

$$
\begin{aligned}
& f_{\Theta \mid \mathrm{A}}(\theta)=\left.\frac{1}{|d \theta / d r|} f_{R \mid A}(r)\right|_{r=\tan \theta} \\
& f_{\Theta \mid \mathrm{A}}(\theta)=\left.\left(1+r^{2}\right) \frac{2}{\pi\left(1+r^{2}\right)}\right|_{r=\tan \theta}
\end{aligned}
$$

Equation (3.80) can be written as

$$
f_{\Theta \mid \mathrm{A}}(\theta)=\frac{2}{\pi}
$$

which implies

$$
f_{\Theta}(\theta)=\frac{1}{2 \pi} \quad \text { for }-\pi \leq \theta \leq \frac{-\pi}{2}
$$

Similarly for $0<X \leq \infty,-\infty \leq Y \leq \infty$ and for $-\infty \leq X<0,-\infty \leq Y \leq 0$, it can be proven [41] that 


$$
\begin{gathered}
f_{\Theta \mid \mathrm{B}}(\theta)=\frac{1}{\pi} \\
f_{\Theta \mid \mathrm{C}}(\theta)=\frac{2}{\pi}
\end{gathered}
$$

Therefore, the unconditional probability density function for the phase $\theta$ can be written as

$$
\begin{gathered}
f_{\Theta}(\theta)=f_{\Theta \mid \mathrm{A}}(\theta) \operatorname{Pr}(A)+f_{\Theta \mid \mathrm{B}}(\theta) \operatorname{Pr}(B)+f_{\Theta \mid \mathrm{C}}(\theta) \operatorname{Pr}(C) \\
=\frac{2}{\pi}\left(\frac{1}{4}\right) I_{\left(-\pi, \frac{\pi}{2}\right)}(\theta)+\frac{1}{\pi}\left(\frac{1}{2}\right) I_{\left(\frac{\pi}{2}, \frac{\pi}{2}\right)}(\theta)+\frac{2}{\pi}\left(\frac{1}{4}\right) I_{\left(\frac{\pi}{2}, \pi\right)}(\theta) \\
= \begin{cases}\frac{1}{2 \pi} & \text { if }-\pi<\theta \leq \pi \\
0 & \text { otherwise }\end{cases}
\end{gathered}
$$

where $I($.$) is the indicator function defined as$

$$
I_{A}(x)=\left\{\begin{array}{lr}
1 & \text { if } x \in A \\
0 & \text { otherwise }
\end{array}\right.
$$

In this section, it was proved that the complex sum of two zero mean IID GRVs gives a complex sum with Rayleigh distributed magnitude and uniformly distributed phase in $(-\pi, \Pi]$. Therefore, this model can be used to simulate the frequency response of a multipath flat Rayleigh faded channel.

\subsubsection{Maximal-Ratio Combining}

As stated in previous sections, many wireless communication systems operate in multipath channels and the performance in a multipath channel is often reduced as compared to an AWGN channel. Diversity techniques can be employed to mitigate the effect of multipath. Diversity simply implies the transmission or reception of multiple copies of the same signal. Diversity can be achieved in time, frequency and space domains. It is assumed that all these diversity receptions are independent each with an independent channel response. [48]

For BPSK, the transmitted baseband symbols are defined as 


$$
\begin{array}{ll}
x_{0}(t)=A e^{0}=A & \text { for } 0 \leq t \leq T_{c} \\
x_{1}(t)=A e^{-i \pi}=-A & \text { for } 0 \leq t \leq T_{c}
\end{array}
$$

where $c T$ is the symbol duration in each time diversity and $A$ is the peak amplitude of the BPSK signal. The energy for each time diversity reception, $E$ , can be given as [49]

$$
E=\int_{0}^{T_{c}}\left|x_{k}(t)\right|^{2} d t=A^{2} T_{c} \quad \text { for } k=0,1
$$

For time diversity, it is assumed that each received diversity signal passes through an independent faded channel and can be represented as

$$
r_{1}(t)=h_{l} e^{i \theta_{l}} x_{k}(t)+n_{l}(t) \quad \text { for } 0 \leq t \leq T_{c}, k=0,1
$$

where $l$ represents the number of the diversity reception and $n_{l}(t)$ is the complex valued AWGN with the circularly symmetric probability density function. The power spectral density function for $n_{l}(t)$ is

$$
S_{n n}(f)=N_{0}
$$

where the power spectral density functions for the real and imaginary components of $n_{l}(t)$ are

$$
S_{\operatorname{Re}[n] \operatorname{Re}[n]}(f)=S_{\operatorname{Im}[n] \operatorname{Im}[n]}(f)=\frac{N_{0}}{2}
$$

For all the diversity receptions, the received signal can be written as

$$
r(t)=\sum_{l=1}^{L} h_{l} e^{-i \theta_{l}} x_{k}(t)+n_{l}(t)
$$

The random variable $Y_{l, k}$ after the correlation receiver can be represented as [41]

$$
\begin{gathered}
Y_{l, k}=A \int_{0}^{T_{c}}\left(h_{l} e^{-i \theta_{l}} x_{k}(t)+n_{l}(t)\right) d t \\
Y_{l, k}=\left\{\begin{array}{l}
A^{2} T_{c} h_{l} e^{-i \theta_{l}}+N_{l} \text { for } k=0 \\
-A^{2} T_{c} h_{l} e^{-i \theta_{l}}+N_{l} \text { for } k=1
\end{array}\right.
\end{gathered}
$$


where $N_{l}$ is a zero-mean complex Gaussian random variable with circularly symmetric probability density function which represents the noise component and can be given as

$$
N_{l}=A \int_{0}^{T_{c}} n_{1}(t) d t
$$

Since the integrator is a filter with frequency response $H_{\text {int }}(f)$ and impulse response

$$
h_{\text {int }}(f)= \begin{cases}1 & \text { if } 0 \leq t \leq T_{b} \\ 0 & \text { itherwise }\end{cases}
$$

Then, the variance of $N_{l}$ can be calculated using Paresval's Theorem and Equations (3.90) and (3.95)

$$
\begin{aligned}
\sigma_{N_{l}}^{2} \equiv E\left\{\left|N_{l}^{2}\right|\right\} & =A^{2} \int_{-\infty}^{+\infty}\left|H_{\text {int }}(f)\right|^{2} S_{n n}(f) d f \\
= & A^{2} \int_{-\infty}^{+\infty} h_{\text {int }}^{2}(t) N_{0} d f \\
= & A^{2} N_{0} T_{b}=E N_{0}
\end{aligned}
$$

Substituting Equation (3.88) into Equation (3.93) yields

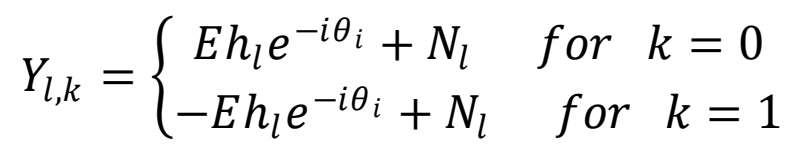

It is assumed that the exact channel state information (CSI) is known at the receiver end. The complex conjugate of the CSI is multiplied by the random variables $Y_{l, k}$ to produce the random variables $Z_{l, k}$. Thus, the phase shift in the channel is compensated and the value of the mean of the random variables $Z_{l, k}$ is proportional to the signal power. Therefore, a strong received signal carries a larger weight than a weak received signal. All the diversity receptions are added to form the random variable $Z_{k}$. This optimum combiner is called the maximal ratio combiner (MRC) [49]. Figure 3.9 illustrates the MRC for BPSK with time diversity. 


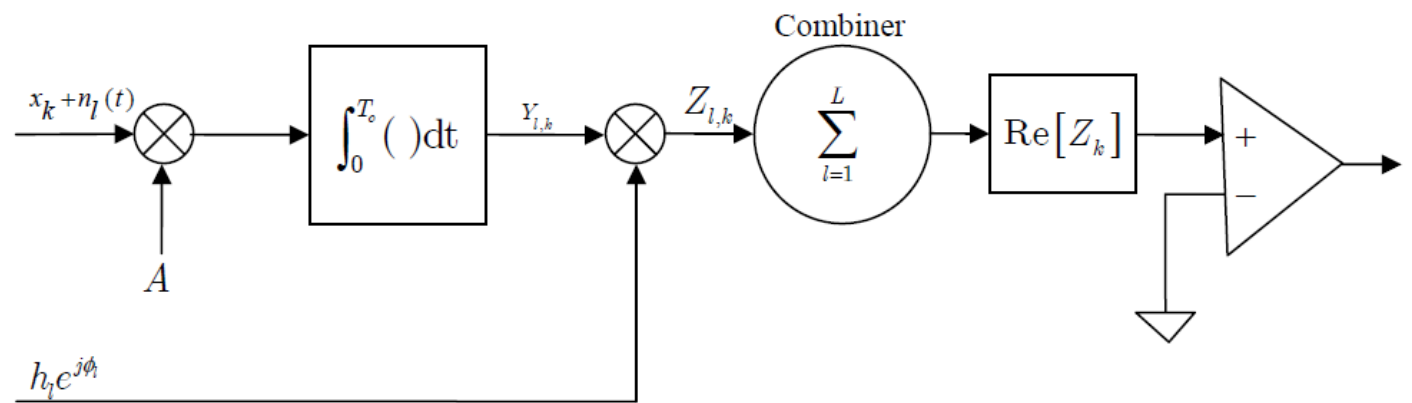

Figure 3.9: MRC for BPSK with time diversity [93, 100].

After maximal ratio combining, the random variable $Z_{k}$ can be written as

$$
Z_{k}=\left\{\begin{array}{cc}
\sum_{l=1}^{l}\left[E h_{l}^{2}+h_{l} e^{i \theta_{l}} N_{l}\right] & \text { for } k=0 \\
-\sum_{l=1}^{l}\left[E h_{l}^{2}+h_{l} e^{i \theta_{l}} N_{l}\right] & \text { for } k=1
\end{array}\right.
$$

The real part of , $Z_{k}$ denoted by $\zeta_{k}=\operatorname{Re}\left\{Z_{k}\right\}$ is fed to the comparator for comparison with the predefined threshold level. For BPSK, if $\zeta_{k}>0$, the receiver decides $k=0$ was transmitted. If $\zeta_{k}<0$, the receiver decides $k=1$ was transmitted. The decision variable $\zeta_{k}$ can be written as

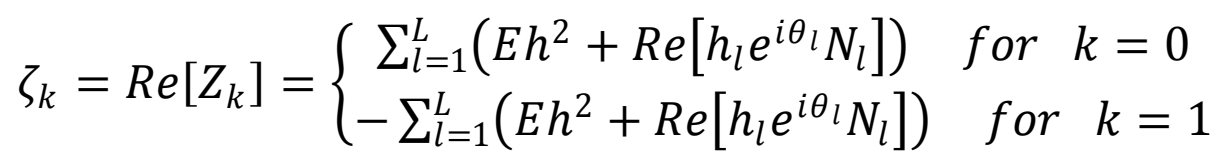

From Equation (3.99), it is evident that the decision variable $\zeta_{k}$ is a Gaussian random variable with conditional mean (conditioned on the value of $\sum_{l=1}^{L} h_{l}^{2}$ )

$$
\overline{\zeta_{k}}=(-1)^{k} E \sum_{l=1}^{L} h_{l}^{2}
$$

Since the $N_{l}$ 's are independent zero-mean complex Gaussian random variables with circularly symmetric probability density function and variance equal to $E N_{0}$, the conditional variance of $\zeta_{k}$ can be expressed as:

$$
\begin{gathered}
\sigma_{\zeta}^{2}=\sigma_{\zeta_{0}}^{2}=\sigma_{\zeta_{1}}^{2} \\
=\sum_{l=1}^{L} \operatorname{Var}\left\{\operatorname{Re}\left(h_{l} e^{i \varphi_{l}} N_{l}\right)\right\}
\end{gathered}
$$




$$
\begin{gathered}
=\sum_{l=1}^{L} h_{l}^{2} \operatorname{Var}\left\{\operatorname{Re}\left(N_{l}\right)\right\} \\
=\frac{1}{2} \sum_{l=1}^{L} h_{l}^{2} \operatorname{Var}\left\{N_{l}\right\} \\
=\frac{E N_{0}}{2} \sum_{l=1}^{l} h_{l}^{2}
\end{gathered}
$$

Assuming the probability of transmitting a " 1 " bit and a " 0 " bit are equal and using the fact that the conditional probabilities of bit error $P_{b \mid k}$ are equal due to the symmetry of the noise probability density function and the zero threshold, it is possible to calculate the conditional probability of bit error as:

$$
\begin{gathered}
P_{b}=\frac{1}{2} P_{b \mid 0}+\frac{1}{2} P_{b \mid 1}=P_{b \mid 0}=P R\left\{\zeta_{0}<0\right\} \\
=\operatorname{Pr}\left\{\frac{\zeta_{0}-\overline{\zeta_{0}}}{\sigma_{\zeta}}<\frac{\overline{\zeta_{0}}}{\sigma_{\zeta}}\right\} \\
=\operatorname{Pr}\left\{\frac{\zeta_{0}-\overline{\zeta_{0}}}{\sigma_{\zeta}}>\frac{\overline{\zeta_{0}}}{\sigma_{\zeta}}\right\} \\
=Q\left(\frac{\overline{\zeta_{0}}}{\sigma_{\zeta}}\right) \\
=Q\left(\sqrt{\frac{2 E}{N_{0}} \sum_{l=1}^{L} h_{l}^{2}}\right)
\end{gathered}
$$

Where $Q(x)$ is defined as [41]

$$
Q(x)=\frac{1}{\sqrt{2 \pi}} \int_{x}^{\infty} e^{\frac{u^{2}}{2} d u}
$$


Let $\gamma$ be defined as

$$
\gamma=\frac{E}{N_{0}} \sum_{l=1}^{L} h_{l}^{2}
$$

Substituting Equation (3.103) into (3.102), it is possible to rewrite the conditional probability of bit error as a probability of bit error conditioned on

$$
P_{b}(\gamma)=Q(\sqrt{2 \gamma})
$$

The average probability of bit error can be obtained by taking the expectation of $P_{b}(\gamma)$ with respect to random variable $\gamma[48,49]$. The average probability of bit error can be written as

$$
\begin{gathered}
\overline{P_{b}}=E\left[P_{b}(\gamma)\right] \\
=\int_{-\infty}^{\infty} P_{b}(\gamma) f_{\Gamma}(\gamma) d \gamma
\end{gathered}
$$

where $f_{\Gamma}(\gamma)$ is the probability density function for $\gamma$. Similarly, $\gamma_{l}$ can be written as

$$
\gamma_{l}=\frac{E h_{l}^{2}}{N_{0}}
$$

The probability distribution function of $h_{l}^{2}$ was derived in the last section. Taking $h_{l}^{2}=v_{l}$, Equation (3.60) can be represented as

$$
f_{v_{1}}\left(v_{1}\right)=\frac{1}{2 \sigma^{2}} e^{-v_{1} / 2 \sigma^{2}} u\left(v_{1}\right)
$$

The average SNR per diversity reception can be written as

$$
\begin{aligned}
& \overline{\gamma_{l}}=E\left[\gamma_{l}\right]=E\left[\frac{E h_{l}^{2}}{N_{0}}\right] \\
& =\frac{E}{N_{0}} E\left[h_{l}^{2}\right]=\frac{E}{N_{0}} E\left[v_{1}\right]
\end{aligned}
$$


The expectation can be evaluated as

$$
\begin{gathered}
E\left[v_{1}\right]=\int_{0}^{\infty} v_{1} f_{v_{1}}\left(v_{1}\right) d v_{1} \\
=\int_{0}^{\infty} v_{1} \frac{1}{2 \sigma^{2}} e^{-v_{1} / 2 \sigma^{2}} d v_{1}=2 \sigma^{2}
\end{gathered}
$$

Now the Equation (3.109) can be written as

$$
\gamma_{l}=\frac{2 E \sigma^{2}}{N_{0}}
$$

The characteristic function of $V_{l}$ can be written as [41, 49]

$$
F_{v_{1}}(\omega)=\frac{1}{\left(1-i 2 \sigma^{2} \omega\right)}
$$

If $L$ IID random variables are added, then the probability density function of the sum is the $L$ fold convolution of the probability density function of the single random variable. Therefore, the characteristic function of the sum is the characteristic function of the single random variable raised to the power of $L$ $[49,51]$. Therefore, the characteristic function for $V=\sum_{l=1}^{L} V_{1}$ is

$$
F_{v_{1}}(\omega)=\frac{1}{\left(1-i 2 \sigma^{2} \omega\right)^{L}}
$$

The probability density function of $V$ is the inverse Fourier transform of the characteristic function and can be written as [41]

$$
f_{v}(v)=\frac{v^{L-1}}{2^{L} \sigma^{2 L}(L-1) !} e^{-v / 2 \sigma^{2}} u(v)
$$

Consistent with Equation (3.104), it is possible to write

$$
\gamma=\frac{E v}{N_{0}}
$$

And

$$
\frac{d \gamma}{d v}=\frac{E}{N_{0}}
$$


Now, the probability distribution function for $\gamma$ can be given as

$$
\begin{gathered}
f_{\Gamma}(\gamma)=\left.\frac{1}{\frac{E}{N_{0}}} \frac{v^{L-1}}{2^{L} \sigma^{2 L}(L-1) !} e^{-v / 2 \sigma^{2}}\right|_{v=\frac{\gamma}{E / N_{0}}} \\
f_{\Gamma}(\gamma)=\frac{\gamma^{L-1}}{\left(\frac{E 2 \sigma^{2}}{N_{0}}\right)^{L}(L-1) !} e^{\frac{\gamma}{E 2 \sigma^{2}}}
\end{gathered}
$$

Substituting Equation (3.111) into Equation (3.117) yields

$$
f_{\Gamma}(\gamma)=\frac{\gamma^{L-1}}{(\bar{\gamma})^{L}(L-1) !} e^{\frac{\gamma}{\bar{\gamma}}} u(\gamma)
$$

Now, substituting Equation (3.105) and (3.118) into Equation (3.106), the average probability of bit error can be represented as [41]

$$
\overline{P_{b}}=\int_{0}^{\infty} Q(\sqrt{2 \gamma}) \frac{\gamma^{L-1}}{(\bar{\gamma})^{L}(L-1) !} e^{\frac{\gamma}{\bar{\gamma}_{l}}} d \gamma
$$

The solution for Equation (3.119) has been given in [49] as

$$
\overline{P_{b}}=\left[\frac{(1-u)}{2}\right]^{L} \sum_{l=0}^{L-1}\left(\begin{array}{c}
L-1+l \\
l
\end{array}\right)\left[\frac{(1+u)}{2}\right]^{l}
$$

where $u$ is defined as

$$
u=\sqrt{\frac{\bar{\gamma}}{1+\bar{\gamma}}}
$$

In time diversity, the total bit energy received is proportional to the number of diversity receptions and is represented as

$$
E_{b}=L E
$$


where $L$ is the number of diversity reception and $E$ is the energy per diversity reception. Substituting Equation (3.122) into Equation (3.109), $\bar{\gamma}_{l}$ can be written as

$$
\bar{\gamma}_{l}=\frac{2 E_{b} \sigma^{2}}{L N_{0}}
$$

Similarly, in the case of space diversity of order $J$ as illustrated in Figure 3.10, each diversity reception is processed by a separate correlator. The integrating time period in each correlator is $T_{b}$. Therefore, the bit energy per diversity reception can be represented as

$$
E=E_{b}
$$

Now, the average SNR per diversity reception $\bar{\gamma}_{l}$ can be written as

$$
\bar{\gamma}_{l}=\frac{2 E_{b} \sigma^{2}}{N_{0}}
$$

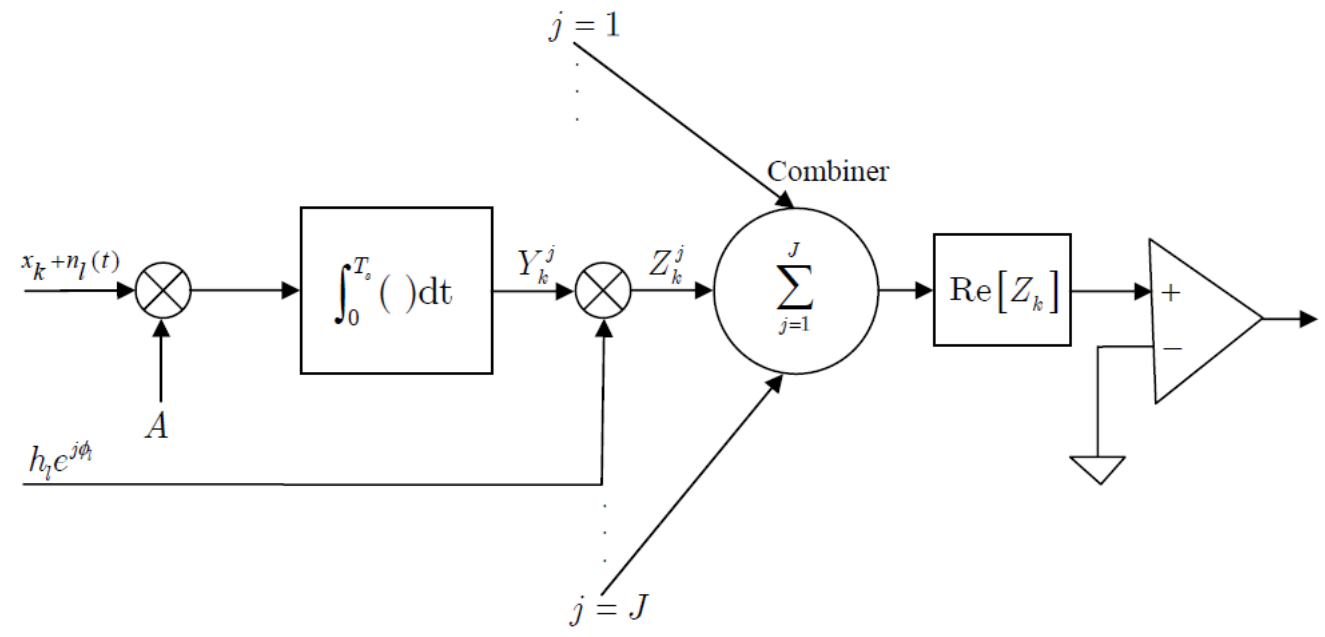

Figure 3.10: MRC for BKSP for Space Diversity [41, 48]

In this thesis, the performance of MDDM in MISO and MIMO systems is compared with the SISO system. In the case of the SISO system, there is no diversity and $L=1$. Thus, the probability of bit error $\overline{P_{b}}$ as per Equation (3.120) is given as $[48,49]$

$$
\overline{P_{b}}=\left[\frac{1-u}{2}\right]
$$


Substituting Equation (3.121) into Equation (3.126) yields

$$
P_{b}=\left[\frac{1}{2}\left(1-\sqrt{\frac{\bar{\gamma}_{l}}{1-\bar{\gamma}_{l}}}\right)\right]
$$

The probability of bit error for a BPSK MISO system in a multipath fading channel as given in Equation (3.127) will be compared with the simulated and theoretical probability of bit error for MDDM MISO and MIMO systems.

\subsection{Summary}

In this chapter, the fundamentals of SISO, SIMO and MISO systems were introduced to facilitate better understanding of MIMO systems. To approach the maximum capacity of MIMO channels, space time codes can be designed in conjunction with the modulation techniques to incorporate diversity. Orthogonal frequency division multiplexing (OFDM) can be used to mitigate the frequency selectivity of the channel. OFDM utilizes the discrete Fourier transform (DFT) of the baseband signal. The multicarrier cyclic delay diversity technique can be implemented by exploiting the cyclic shift property of the DFT. A baseband model for the flat Rayleigh fading channel was derived for simulation. Lastly, the MRC as an optimum diversity combining technique was discussed. The next chapter discusses the design of the simulated MDDM transmitters and receivers. 


\section{Chapter 4}

\section{MULTICARRIER DELAY DIVERSITY MODULATION TRANSMITTER AND RECEIVER MODELS}

This chapter introduces the implementation of the multicarrier delay diversity modulation scheme at the block level. The basic design of the transmitter and receiver is discussed briefly. In this thesis, the MDDM scheme is implemented using BPSK modulation. The inclusion of forward error correction (FEC) and higher bandwidth efficient modulation schemes can be considered for future work. The next chapter illustrates the simulation and analysis of the MDDM transmitter and receiver.

\subsection{The Multicarrier Delay Diversity Modulation Scheme}

The scheme of implementing delay diversity with OFDM for MIMO systems was recommended for flat fading channels in [90]. The cyclic delay diversity approach with OFDM was suggested for the frequency selective fading channel in [39]. In this research, cyclic delay diversity with OFDM is investigated. The implementation of multicarrier delay diversity in MIMO systems has been discussed in the previous chapter. For modeling purposes, the $2 * 2$ MIMO configuration is discussed in this chapter. For simulation and analysis, the number of receive antennas varies from one to three. To facilitate better understanding and precise representation of signals from the transmit antenna to the receive antenna, the following notations will be used:

- $X_{k}$ represents the BPSK symbol at $k^{- \text {th }}$ interval before the multicarrier delay diversity modulation and after demodulation

- $X_{k}^{l}$ represents the BPSK symbol at $k^{\text {th }}$ interval from the transmitting antenna $l$ after multicarrier delay diversity delay modulation

- $x_{m}^{l}$ represents the signal $x$ to be transmitted from transmit antenna $l$ in the $m$ th transmission interval,

- $r_{m}^{j}$ represents the received signal at receive antenna $j$ at the $m^{\text {th }}$ time interval, 
- $h^{l j}$ represents the channel response from transmit antenna $l$ to receive antenna $j$,

- $n^{j}$ represents the AWGN at receive antenna $j$, and

- asterisk ( $)^{*}$ represents the complex conjugate.

\subsection{MDDM Transmitter}

The multicarrier delay diversity scheme will be simulated with binary phase shift keying modulation. A block diagram of the MDDM transmitter with two transmitting antennas is shown in Figure 4.1. The function of each block is explained briefly in the following discussion.

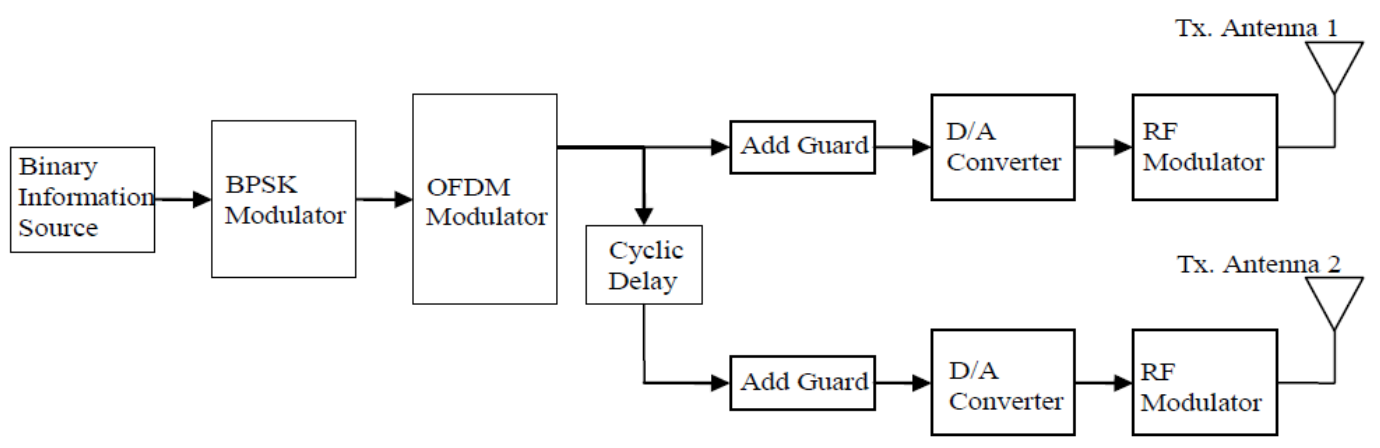

Figure 4.1: Block diagram of MDDM transmitter [34]

\subsubsection{Binary Information Source and $M$ PSK Modulator}

The binary information source generates bits 0 and 1 with equal probabilities

$$
\operatorname{Pr}[0]=\operatorname{Pr}[1]=1 / 2
$$

Then, the bits are mapped to $I$ and $Q$ symbol coordinates by the $M$ PSK modulator. The equivalent lowpass (i.e. complex envelope) $M$ PSK symbols can be represented as

$$
X_{k}(t)=A e^{\frac{j 2 \pi(m-1)}{M}} P_{T}(t) \quad \text { for } m=1,2, \ldots \ldots, M
$$

where $A$ is the amplitude of the signal, $M$ is the number of possible phases (for BPSK $M=2$, for QPSK $M=4$ ), $T$ is the symbol duration, and $P_{T}(t)$ denotes 


$$
P_{T}(t)= \begin{cases}1 & \text { for } 0 \leq t \leq T \\ 0 & \text { otherwise }\end{cases}
$$

\subsubsection{OFDM Modulator}

Mapped symbols are fed to the OFDM modulator block. In this block, first the serial input data stream is converted to parallel. Then, the IFFT operation is performed to realize OFDM as discussed in the last chapter. The FFT size denotes the number of subcarriers. Figure 4.2 illustrates the OFDM modulator. [34]

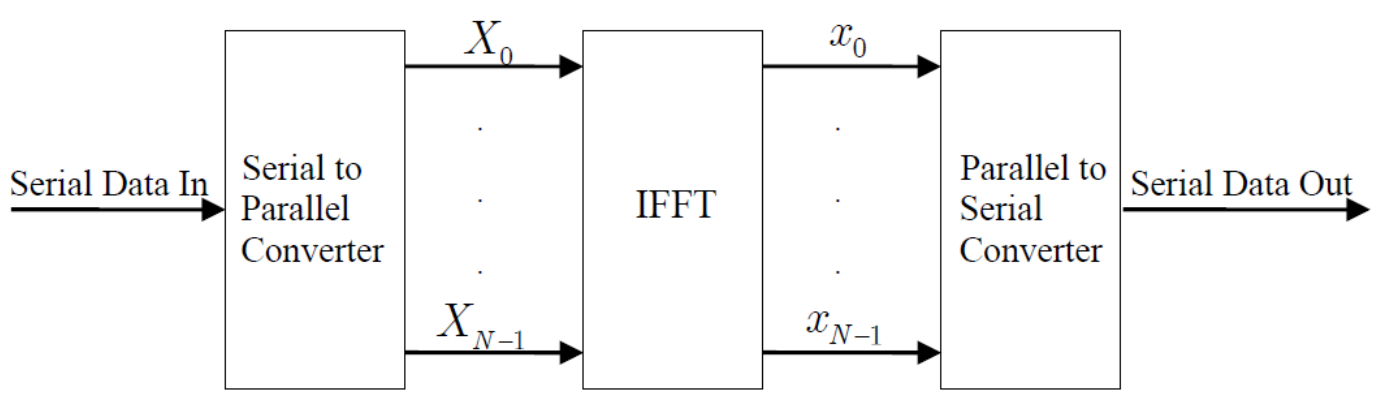

Figure 4.2: OFDM modulator

In this thesis, the number of subcarriers is 256 to simulate OFDM, in accordance with the IEEE 802.16a standard. In this standard, three types of subcarriers are defined: data carriers for information symbol transmission, pilot carriers for the estimation of CSI and guard carriers (null carriers) placed on both sides of the frequency spectrum to avoid intercarrier interference from neighboring frequency bands.

\subsubsection{Cyclic Delay Addition}

After the IFFT operation, the symbols $n x$ are cyclically shifted to realize a cyclic delay of one information symbol period. For transmitting antenna 1, there is no delay and $D=0$. For each subsequent antenna, the cyclic delay is increased one information symbol period. The signal after cyclic delay is given as

$$
x_{n}^{1}=x_{(n-D) N} \quad \text { where } l=1,2, \ldots ., L \quad \text { and } \quad D=l-1
$$

\subsubsection{Guard Interval (Cyclic Prefix) Addition}


As discussed in the previous chapter, a guard interval at the beginning of each OFDM symbol is added to mitigate the effect of the multipath channel. The length of the guard interval, $T_{G}$ in the form of a cyclic prefix, is chosen to be greater than or equal to the anticipated delay spread of the channel. The addition of a guard interval is an overhead at the cost of data rate. The guard length is specified with respect to the OFDM symbol period $T_{S}$ and is also referred to as guard ratio $T_{G} / T_{S}$. The addition of the guard interval has been illustrated in Figure 3.7. For example, in the IEEE 802.16 wireless standard, of the guard ratios is chosen to be $1 / 32$. The FFT block size is 256 . Therefore, the guard length is to be 8 . The cyclic prefix constitutes the last eight samples of the IFFT output and it is concatenated to the beginning of the OFDM symbol sequence. Now, the sequence with added guard interval for each transmitting antenna can be represented as

$$
\begin{gathered}
x_{(n+G)}^{1}=[x[248] x[249] \ldots x[255] \\
x_{(n+G)}^{2}=\left[\begin{array}{llll}
x[247] & x[248] \ldots x[254] & x[255] x[0] & x[1] \ldots x[254]
\end{array}\right.
\end{gathered}
$$

\subsection{Digital to Analog Conversion and RF Modulation}

The IFFT operation, the cyclic delay operation and the guard interval operations are performed in the discrete time domain. For transmission purposes, these discrete symbols are converted to an analog signal using a digital to analog (D/A) converter. After digital to analog conversion, these symbols are continuous time baseband OFDM symbols. Then, these symbols are up converted to the transmission radio frequency. After requisite amplification to the desired power level, these symbols are transmitted from the respective antennas. In this thesis, the simulation is performed at the discrete time baseband level and no digital to analog or RF modulation is implemented.

\subsection{MDDM Receiver}

The multicarrier delay diversity scheme can be employed with any number of receive antenna without changing the transmitted space time code. In this section, the receiver design for two receive antennas is discussed. It is assumed that the signals received at both receiving antennas are independent of each other due to independent channel responses and no antenna correlation. The MDDM receiver has almost the same structure as the MDDM transmitter but the operations are performed in reverse order. The 
MDDM receiver design is illustrated in Figure 4.3. At the receiving antenna, the cyclically time delayed OFDM symbols are received after passing through the channel with the AWGN and can be represented as

$$
\begin{gathered}
r_{(n+G)}^{1}=A\left(h^{11} * x_{(n+G)}^{1}+h^{21} * x_{(n+G)}^{2}\right)+n^{1} \text { for } n \\
=1,1,2, \ldots, N+G-1 \\
r_{(n+G)}^{2}=A\left(h^{12} * x_{(n+G)}^{1}+h^{22} * x_{(n+G)}^{2}\right)+n^{2} \text { for } n \\
=1,1,2, \ldots, N+G-1
\end{gathered}
$$

where * represents circular convolution (due to the circular guard interval) [35].

After RF demodulation and analog to digital (A/D) conversion, the OFDM symbol received by each receiving antenna is in the discrete time domain. The guard interval added at the transmitter is removed. Then, this signal is fed to the OFDM demodulator as a baseband signal. In the OFDM demodulator block, the received OFDM symbol is converted from serial to parallel for the FFT operation. The OFDM demodulator is illustrated in Figure 4.4. After the FFT operation as defined in Equation (3.29), the signal can be represented as

$$
\begin{aligned}
& R_{k}^{j}=F F T\left\{r_{n}^{j}\right\}=\sum_{n=0}^{255}\left(\sum_{l=1}^{2}\left(h^{l j} * x^{l}\right)+n^{j}\right) e^{j 2 \pi k n / 255} \quad \text { for } n= \\
& 0,1,2, \ldots, 255(4.8)
\end{aligned}
$$

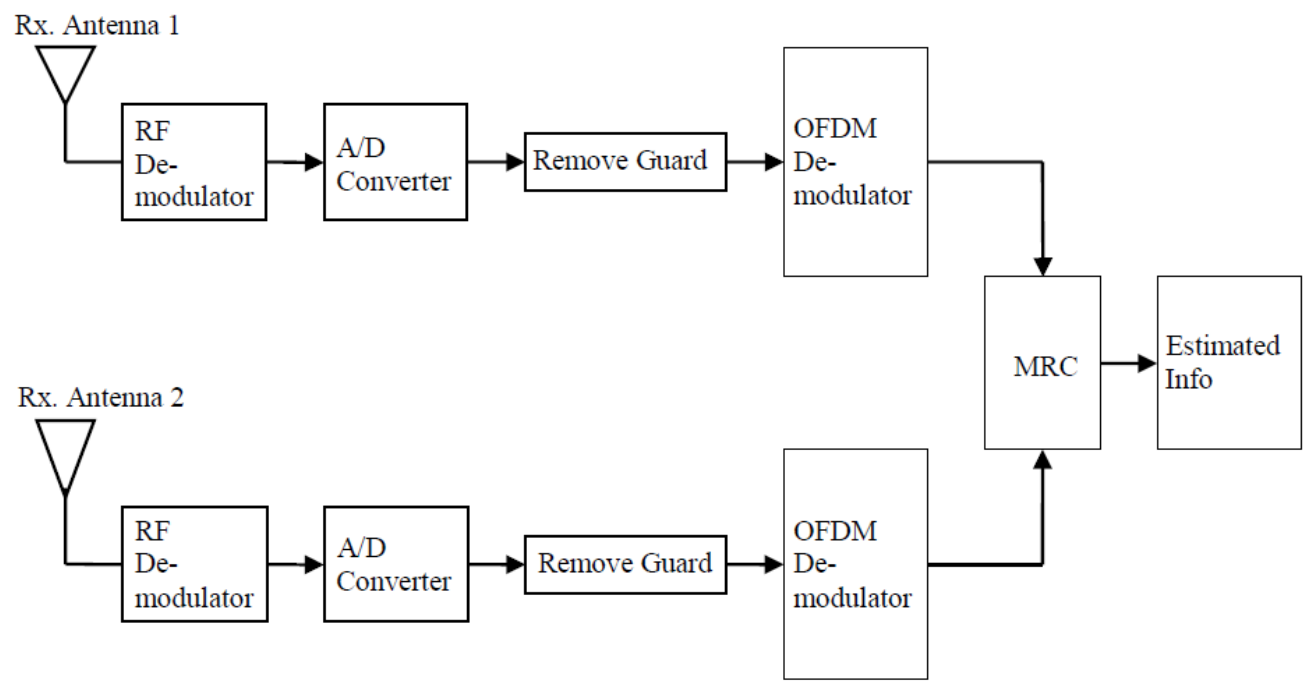

Figure 4.3: Block diagram of MDDM receiver 


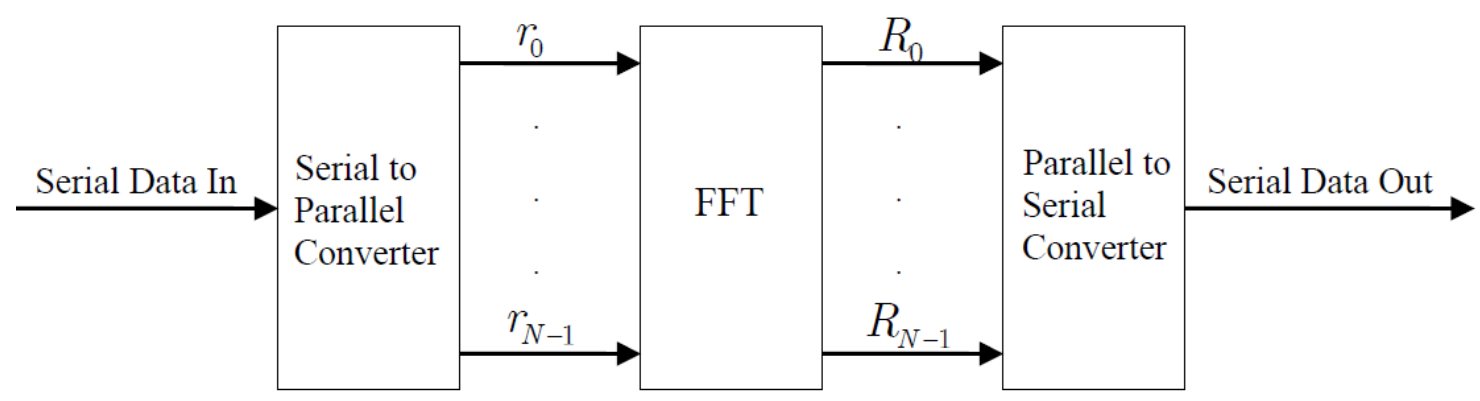

Figure 4.4: OFDM demodulator

Using the linearity property of the discrete Fourier transform, Equation (4.8) can be represented as

$$
\begin{aligned}
& R_{k}^{1}=H^{11} X_{k}^{1}+H^{21} X_{k}^{2}+N_{k}^{1} \quad \text { for } k=0,1,2, \ldots, 255 \\
& R_{k}^{2}=H^{12} X_{k}^{1}+H^{22} X_{k}^{2}+N_{k}^{2} \text { for } k=0,1,2, \ldots, 255
\end{aligned}
$$

where $H^{j l}$ is the frequency response of the channel from transmit antenna $l$ to receive antenna $j$ and $N^{j}$ is the AWGN at receive antenna $j$ in the frequency domain. Using the circular time shift property of the discrete Fourier transform as defined in Equation (3.31), Equation (4.9) can be written as

$$
\begin{array}{ll}
R_{k}^{1}=H^{11} X_{k}^{1}+H^{21} X_{k}^{1} e^{-i 2 \pi k D / N}+N^{1} & \text { for } k=0,1,2, \ldots, 255 \\
R_{k}^{2}=H^{12} X_{k}^{1}+H^{22} X_{k}^{1} e^{-i 2 \pi k D / N}+N^{2} & \text { for } k=0,1,2, \ldots, 255 \\
R_{k}^{1}=H^{11} X_{k}^{1}+H^{21} X_{k}^{1} e^{-i 2 \pi k D / N}+N^{1} & \text { for } k=0,1,2, \ldots, 255 \\
R_{k}^{2}=H^{12} X_{k}^{1}+H^{22} X_{k}^{1} e^{-i 2 \pi k D / N}+N^{2} & \text { for } k=0,1,2, \ldots, 255
\end{array}
$$

where $D=1$ is the cyclic time delay induced at transmit antenna two. Now, the effective channel response at the receive antenna $j$ is defined as

$$
H_{k}^{j}=H_{k}^{1 j}+H_{k}^{2 j} e^{2 \pi k / N}
$$

Substituting Equation (4.11) into Equation (4.10), the signal at each receiving antenna can be written as 


$$
\begin{gathered}
R_{k}^{l}=H_{k}^{1} X_{k}^{1}+N_{k}^{1} \\
R_{k}^{2}=H_{k}^{2} X_{k}^{1}+N_{k}^{2}
\end{gathered}
$$

Equation (4.12) shows clearly the space diversity receptions at the receiving end. They are combined by using the optimum maximal ratio combining receiver. MRC for space diversity has already been discussed in the previous chapter and illustrated in Figure 3.9. It is assumed that perfect CSI is known at the receiver end. In MRC, the received signal is multiplied with the complex conjugate of CSI and all diversity receptions are added to form the decision variable. The outputs of the integrators of the MRC for each diversity reception can be represented as

$$
\begin{gathered}
Y_{k}^{1}=A T_{b} H_{k}^{1} X_{k}+N_{k}^{1} \\
Y_{k}^{2}=A T_{b} H_{k}^{2} X_{k}+N_{k}^{2} \\
Y_{k}^{1}=\frac{E_{b} H_{k}^{1} X_{k}}{A}+N_{k}^{1} \\
Y_{k}^{2}=\frac{E_{b} H_{k}^{2} X_{k}}{A}+N_{k}^{2}
\end{gathered}
$$

where $E_{b}$ is the average energy per bit, $N_{k}^{1}=A T_{b} N_{k}^{1}$ and $N_{k}^{2}=A T_{b} N_{k}^{2}$

After the integrator stage, the random variable is multiplied by the complex conjugate of CSI and the resulting random variable can be represented as

$$
\begin{gathered}
Z_{k}^{1}=\frac{E_{b} H_{k}^{1}\left(H_{k}^{1}\right) * X_{k}}{A}+\left(H_{k}^{1}\right) * N_{k}^{1} \\
Z_{k}^{2}=\frac{E_{b} H_{k}^{2}\left(H_{k}^{2}\right) * X_{k}}{A}+\left(H_{k}^{2}\right) * N_{k}^{2} \\
Z_{k}^{1}=\frac{E_{b}\left|H_{k}^{1}\right|^{2} X_{k}}{A}+\left(H_{k}^{1}\right) * N_{k}^{1}
\end{gathered}
$$




$$
Z_{k}^{2}=\frac{E_{b}\left|H_{k}^{2}\right|^{2} X_{k}}{A}+\left(H_{k}^{2}\right) * N_{k}^{2}
$$

The decision variable $Z_{k}$ at the output of combiner stage can be expressed as

$$
\begin{gathered}
Z_{k}=Z_{k}^{1}+Z_{k}^{2} \\
Z_{k}=E\left(\left|H_{k}^{1}\right|^{2}+\left|H_{k}^{2}\right|^{2}\right) \frac{X_{k}}{A}+\left(H_{k}^{1}\right) * N_{k}^{1}+\left(H_{k}^{2}\right) * N_{k}^{2}
\end{gathered}
$$

For the final decision of the estimated received data, the decision variable is compared with predetermined threshold levels. For the case of BPSK, the real part of $Z_{k}$ is compared with a threshold level of zero to decide each received binary bit. $[35,48]$

\subsection{Summary}

This chapter discussed the designs of the MDDM transmitter and receiver at the block level. The function of each block was discussed with the representation of signals before and after each block operation. During the discussion, two assumptions were made, i.e., perfect knowledge of CSI at the receiver and constant channel response for the duration of the OFDM symbol. The next chapter analyzes the MDDM transmitter and receiver model for the AWGN channel without flat fading. 


\section{Chapter 5}

\section{ANALYSIS AND SIMULATION OF MULTICARRIER DELAY DIVERSITY MODULATION SCHEME}

In this chapter, the performance (bit error probability) analysis and simulation of MISO and MIMO systems with multicarrier delay diversity is presented. The MDDM transmitter and receiver as described in Chapter IV are simulated in Matlab. The system is analyzed with an AWGN channel without rayleigh fading. The analytical and simulated results are compared with a SISO BPSK system. To establish a fair comparison of the SISO system and the MIMO system with MDDM, the power transmitted for both systems is assumed equal. To facilitate better understanding and precise representation of signals from transmit to receive antenna, this thesis will adhere to the following notation:

- $E_{b}$ represents the average energy per bit at the receiver of the SISO system,

- $E_{S}^{\prime}$ represents the average energy per MIMO BPSK symbol per receiving antenna,

- $T_{s}$ represents the OFDM symbol duration,

- $T_{S}^{\prime}$ represents the MIMO BPSK symbol duration and $T_{s}^{\prime}=T_{b}$

- $\zeta_{k}$ represents the real part of decision variable $Z_{k}$.

Energy per bit is an important parameter for comparison and is defined as

$$
E_{b}=\int_{0}^{T_{b}}\left|X_{k}\right|^{2} d t=A^{2} T_{b}
$$

where $k$ is the time index of the BPSK symbol (before serial to parallel and after parallel to serial conversions) and $X_{k}$ is the BPSK modulated symbol as defined in Table 1. 


\begin{tabular}{|c|c|}
\hline Input bit at time $k$ & Output Symbol $X_{k}$ \\
\hline 0 & $\mathrm{~A}$ \\
\hline 1 & $-\mathrm{A}$ \\
\hline
\end{tabular}

Table 1: the BPSK modulated symbol scheme [41]

For simulation and analysis, the amplitude $A=1$ is maintained. Equation (5.1) shows that the energy per bit for BPSK modulation is the same for bit 0 and bit 1 . Thus the transmitted power for the SISO system can be written as

$$
P_{\text {SISO }}=\frac{E_{b}}{T_{b}}=A^{2}
$$

The probability of bit error $P_{b}$ for a baseband equivalent SISO BPSK system in discrete domain for correlation demodulator can be represented as [48, 49]

$$
P_{b}=Q\left(\frac{\bar{Z}^{+}}{\sigma_{Z}}\right)
$$

In this case $\bar{Z}^{+}$is represented as

$$
\bar{Z}^{+}=A
$$

And

$$
\sigma_{Z}^{2}=\frac{N_{0}}{2 T_{b}}
$$

where $\frac{N_{0}}{2}$ represents the two sided noise power spectral density for the real part of the AWGN. Substituting Equation (5.4) and Equation (5.5) into Equation (5.3) yields,

$$
P_{b}=Q\left(\frac{\sqrt{2 T_{b}} A}{\sqrt{N_{0}}}\right)=Q\left(\sqrt{\frac{2 E_{b}}{N_{0}}}\right)
$$

The bit error probability of the SISO system as given by Equation (5.6) will serve as a benchmark for comparison of the MISO and MIMO systems with MDDM in the AWGN channel.

In this thesis the number of transmit antennas is two and both the transmit antennas transmit equal power. The total energy per bit transmitted for MISO and MIMO systems using BPSK symbols can be defined as 


$$
E_{s}^{\prime}=P^{\prime} T_{s}^{\prime}
$$

And

$$
P_{\text {SISO }}=P_{\text {MISO }}=P_{M I M O}
$$

Therefore, the energy transmitted per antenna $P^{\prime}$ can be given as

$$
P^{\prime}=\frac{P_{S I S O}}{L}=\frac{P_{S I S O}}{2}
$$

Now, Equation (5.7) is rewritten as

$$
\begin{array}{r}
E_{S}^{\prime}=P^{\prime} T_{s}^{\prime}=\frac{P_{S I S O} T_{s}^{\prime}}{2} \\
E_{s}^{\prime}=\frac{E_{b}}{2}
\end{array}
$$

\subsection{Simulation of MDDM Transmitter}

The block diagram of the MDDM transmitter model is shown in Figure 4.1. The MDDM transmitter was simulated in MATLAB with equivalent baseband BPSK in the discrete time domain. The simulation was implemented with one sample for each BPSK symbol. The MDDM transmitter scheme, as mentioned in Chapter IV with two transmitting antennas, was simulated without added guard interval, D/A converter and RF modulator blocks to facilitate the simulations. Equal power was transmitted from both the antennas. To achieve the same total power transmission as that of a single antenna BPSK transmitter, the signal at each branch of MIMO transmitter was multiplied with a gain factor of $g$ for normalization. The transmitted energy per symbol for BPSK is same whether a binary 1 or 0 is transmitted. With the gain factor $g$ the energy per symbol is represented as

$$
E_{s}^{\prime}=\int_{0}^{T_{s}^{\prime}}\left|g X_{k}\right|^{2} d t=g^{2} A^{2} T_{s}^{\prime}
$$

Substituting Equation (5.10) into Equation (5.11) yields

$$
\frac{P_{S I S O} T_{S}^{\prime}}{2}=g^{2} A^{2} T_{S}^{\prime}
$$


Using Equation (5.2), Equation (5.12) can be rewritten as

$$
\begin{aligned}
& g^{2}=\frac{1}{2} \\
& g=\frac{1}{\sqrt{2}}
\end{aligned}
$$

The gain factor $g=1 / \sqrt{2}$ is maintained for all subsequent simulations to transmit the same power as that of a single antenna BPSK system. Thus the effective amplitude of the BPSK modulated signal for each antenna is given as

$$
\left|X_{k}\right|=\frac{A}{\sqrt{2}}=\frac{1}{\sqrt{2}}
$$

\subsection{Simulation of MDDM Receiver}

The block diagram of the MDDM receiver model is shown in Figure 4.3. The MDDM receiver was also simulated in MATLAB with equivalent baseband BPSK in the discrete time domain. The MDDM receiver simulation was implemented as discussed in Chapter IV except for the RF demodulator, the A/D converter and the Remove Guard blocks. The receiver was configured as a MISO system with only one receive antenna and as a MIMO system with two receive antennas and as a MIMO system with three receive antennas. After multicarrier delay diversity demodulation, the space diversity receptions of MIMO systems were combined by using the optimum MRC technique as discussed in Chapter III. After the BPSK correlation demodulator as illustrated in Figure 5.1, $\zeta_{k}$ (the real part of the random variable $Z_{k}$ ) was compared with the threshold level according to Table 2. For analysis and simulation purposes it is assumed that the transmitter and receiver frequencies are synchronized and, in the case of MIMO systems, all the diversity receptions are also synchronized. 


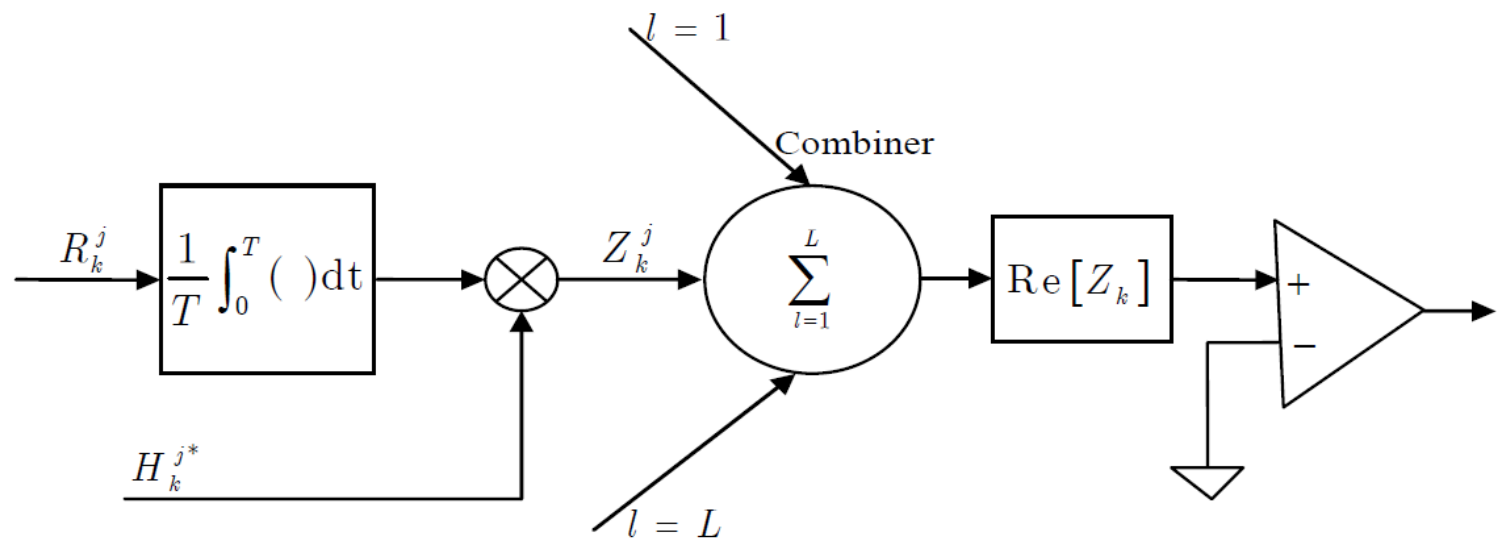

Figure 5.1: BPSK correlation demodulator for MIMO system with MDDM

\begin{tabular}{|l|c|}
\hline & Output Binary Bit \\
\hline$\zeta_{k} \geq 0$ & 0 \\
\hline$\zeta_{k}<0$ & 1 \\
\hline
\end{tabular}

Table 2: Demodulation of BPSK signal [after Ref. 41]

\subsection{Simulation and Performance Analysis of MDDM in AWGN}

The performance of MDDM was first simulated and analyzed in AWGN only. In this simulation and analysis no fading is assumed. This means that each channel response coefficient $h^{l j}$ equals one. A block diagram of the MIMO system in AWGN with three receiving antennas is illustrated in Figure 5.2. Both the MDDM transmitter and receiver are collapsed into one block each to facilitate presentation. In this simulation two gain blocks each with normalizing gain factor of $g$ are shown at the outputs of the transmitter for each transmitting antenna. The AWGN channel blocks add white Gaussian noise to the signal at respective receiving antennas. The noise power is increased progressively with each simulation run to calculate the bit error rate at different signal to noise ratios. Bit error rate is calculated by comparing the input binary data stream at the input to the transmitter $b_{k}$ and output binary data stream at the output of the receiver $\hat{b}_{k}$. 


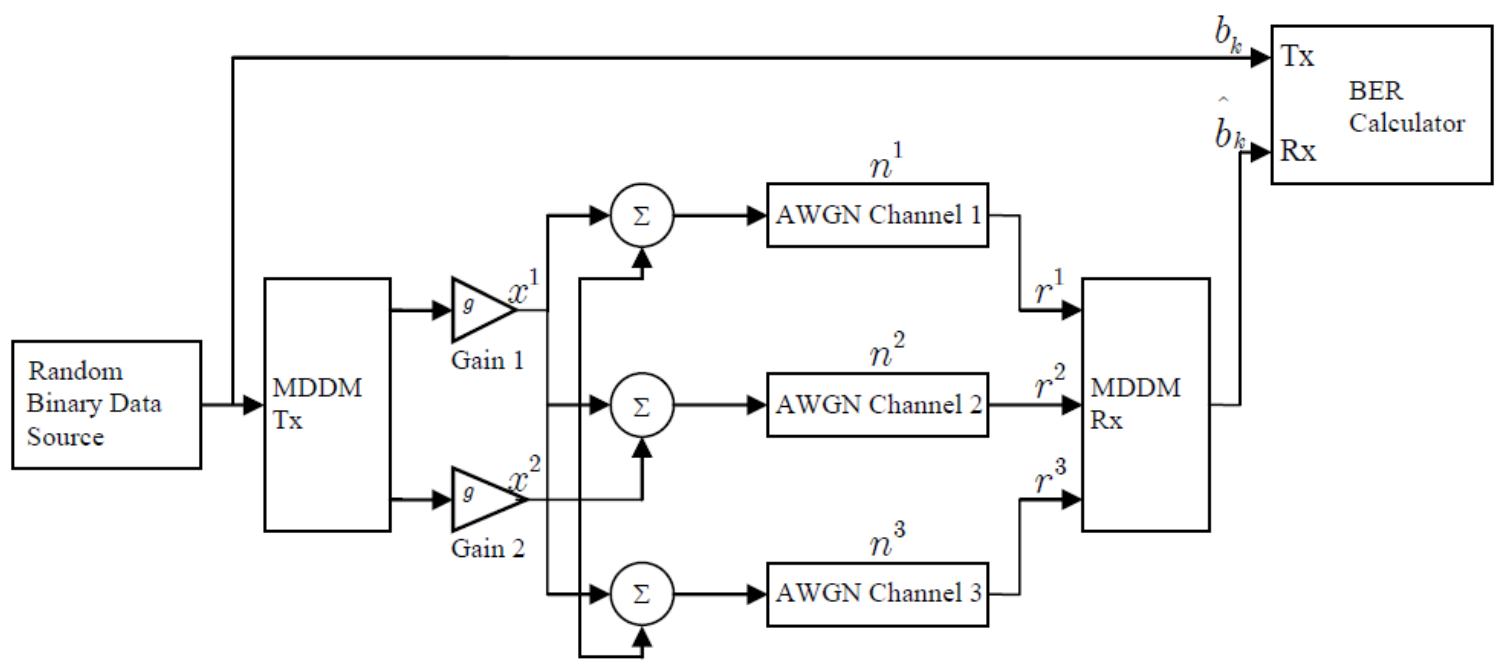

Figure 5.2: Simulation of MDDM MIMO system in AWGN

\subsubsection{Performance Analysis of MISO System with Two Transmitting and One Receiving Antenna}

At the receiving antenna, the received signal $r_{m}$ at time $m$ is given by

$$
r_{m}=x_{m}^{1}+x_{m}^{2}+n_{m}
$$

where $x_{m}^{2}$ is a cyclically delayed signal and is given by Equation (4.5)

$$
x_{m}^{2}=x_{(m-1)_{N}}^{1}
$$

After the FFT operation, the received signal is written as

$$
\operatorname{FFT}\left[r_{m}\right]=\operatorname{FFT}\left[x_{m}^{1}+x_{m}^{2}+n_{m}\right]
$$

Using the linearity property of the discrete Fourier transform [46], Equation (5.17) can be represented as

$$
R_{k}=X_{k}^{1}+X_{k}^{2}+N_{k}
$$

Substituting Equation (3.31) and (5.16) into Equation (5.18) yields

$$
\begin{aligned}
& R_{k}=X_{k}^{1}+X_{k}^{2} e^{-j 2 \pi k / N}+N_{k} \\
& R_{k}=\left(1+e^{-j 2 \pi k / N}\right) X_{k}^{1}+N_{k}
\end{aligned}
$$


where $N$ is the FFT size or the number of subcarriers, $N_{k}$ is AWGN in the frequency domain and $\left(1+e^{-j 2 \pi k / N}\right)$ is the effective channel response for MDDM in AWGN channel. To facilitate the presentation, the phase angle $\phi_{k}$ is defined as

$$
\phi_{k}=2 \pi k / N
$$

Multiplying Equation (5.19) by the complex conjugate of the effective channel response, the result is given by

$$
Z_{k}=\left(1+e^{-j \phi_{k}}\right)\left(1+e^{+j \phi_{k}}\right) X_{k}^{1}+\left(1+e^{+j \phi_{k}}\right) N_{k}
$$

Using Euler's identity

$$
e^{j \theta}=\cos (\theta)+j \sin (\theta)
$$

Equation (5.21) is rewritten as

$$
Z_{k}=\left(2+2 \cos \left(\phi_{k}\right)\right) X_{k}^{1}+\left(1+e^{+j \phi_{k}}\right) N_{k}
$$

Equation (5.23) defines a complex Gaussian random variable $Z_{k}$ due to AWGN. For demodulation of BPSK data, $\zeta_{k}=\operatorname{Re}\left\{Z_{k}\right\}$ is compared with the threshold as given in Table 2. If correlation demodulator conditions are assumed then

$$
\begin{aligned}
& E\left\{Z_{k} \mid \text { "0" was transmitted }\right\} \\
& \qquad \begin{array}{l}
=E\left\{\frac{1}{T_{b}} \int_{0}^{T_{b}}\left[\frac{A}{\sqrt{2}}\left(2+2 \cos \phi_{k}\right)+N_{k}\left(1+e^{+j \phi_{k}}\right)\right] d t\right\} \\
=\left[2+2 \cos \left(\phi_{k}\right)\right] \frac{A}{\sqrt{2}} \\
=\sqrt{2}\left[1+\cos \left(\phi_{k}\right)\right] A
\end{array}
\end{aligned}
$$

Since this expected value is real, it follows that

$$
\bar{\zeta}_{k}^{+}=E\left\{Z_{k} \mid " 0 " w a s \text { sent }\right\}=\sqrt{2\left[1+\cos \left(\phi_{k}\right)\right] A}
$$


The variance of $\zeta_{k}=\operatorname{Re}\left\{Z_{k}\right\}$ is only due to the variance of real part of the noise component $\left(1+e^{+j \phi_{k}}\right) N_{k}[48,49]$ and is represented by $\sigma_{\zeta}^{2}$

$$
\begin{gathered}
\sigma_{\zeta}^{2}=E\left\{\left(\operatorname{Re}\left[\left(1+e^{+j \phi_{k}}\right) N_{k}\right]\right)^{2}\right\}-\left(E\left\{\left(\operatorname{Re}\left[\left(1+e^{+j \phi_{k}}\right) N_{k}\right]\right)\right\}\right)^{2} \\
\sigma_{\zeta}^{2}=E\left\{\left(\operatorname{Re}\left[\left(1+e^{+j \phi_{k}}\right) N_{k}\right]\right)^{2}\right\}
\end{gathered}
$$

where $\mathrm{E}\{\}$ represents the expected value. $\eta_{k}=\operatorname{Re}\left[\left(1+e^{+j \phi_{k}}\right) N_{k}\right]$ is the real part of a complex Gaussian random variable with zero mean. $\eta_{k}$ and $\eta_{n}^{2}$ can be written as

$$
\begin{gathered}
\eta_{k}=\operatorname{Re}\left[\left(1+e^{+j \phi_{k}}\right) N_{k}\right]=\frac{1}{2}\left(\left(1+e^{+j \phi_{k}}\right) N_{k}+\left(1+e^{-j \phi_{k}}\right) N_{k}^{*}\right) \\
\eta_{k}^{2}=\left(\operatorname{Re}\left[\left(1+e^{+j \phi_{k}}\right) N_{k}\right]\right)^{2}=\left(\frac{1}{2}\left(\left(1+e^{+j \phi_{k}}\right) N_{k}+\left(1+e^{-j \phi_{k}}\right) N_{k}^{*}\right)\right)^{2} \\
=\frac{1}{4}\left(\left(1+e^{+j \phi_{k}}\right)^{2} N_{k}^{2}+\left(1+e^{-j \phi_{k}}\right)^{2} N_{k}^{* 2}\right. \\
\left.+2\left(1+e^{-j \phi_{k}}\right)\left(1+e^{+j \phi_{k}}\right) N_{k} N_{k}^{*}\right) \\
=\frac{1}{4}\left(\left(1+e^{+j \phi_{k}}\right)^{2} N_{k}^{2}+\left(1+e^{-j \phi_{k}}\right)^{2} N_{k}^{* 2}+4\left(1+\cos \left(\phi_{k}\right)\right) N_{k} N_{k}^{*}\right)
\end{gathered}
$$

where ( $)^{\star}$ represents the complex conjugate. Substituting Equation (5.27) into Equation (5.26) gives

$$
\begin{gathered}
\sigma_{\zeta}^{2}=E\left\{\frac{1}{4}\left(1+e^{+j \phi_{k}}\right)^{2}\left(N_{k}\right)^{2}+\left(1+e^{-j \phi_{k}}\right)^{2}\left(N_{k}^{*}\right)^{2}\right. \\
\left.+4\left(1+\cos \left(\phi_{k}\right)\right) N_{k} N_{k}^{*}\right\} \\
=\frac{1}{4}\left(1+e^{+j \phi_{k}}\right)^{2} E\left\{\left(N_{k}\right)^{2}\right\}+\left(1+e^{-j \phi_{k}}\right)^{2} E\left\{\left(N_{k}^{*}\right)^{2}\right\} \\
+4\left(1+\cos \left(\phi_{k}\right)\right) E\left\{N_{k} N_{k}^{*}\right\}
\end{gathered}
$$


As discussed earlier, $N_{k}$ is a complex Gaussian random variable with zero mean, therefore

$$
E\left\{\left(N_{k}\right)^{2}\right\}=E\left\{\left(N_{k}^{*}\right)^{2}\right\}=0
$$

Substituting Equation (5.29) into Equation (5.28) gives

$$
\sigma_{\zeta}^{2}=\left\{1+\cos \left(\phi_{k}\right)\right\} E\left\{N_{k} N_{k}^{*}\right\}
$$

Using Equation (3.29), $N_{k}$ is given as

$N_{k}=\sum_{m=0}^{N-1} n_{m} e^{-j 2 \pi k m / N}(5.31)$

Substituting Equation (5.31) into Equation (5.30) yields

$$
\begin{gathered}
\sigma_{\zeta}^{2}=\left[1+\cos \left(\phi_{k}\right)\right] E\left\{\sum_{m=0}^{N-1} n_{m} e^{-j 2 \pi k m / N} \sum_{l=0}^{N-1} n_{l}^{*} e^{+2 \pi k m / N}\right\} \\
=\left[1+\cos \left(\phi_{k}\right)\right] \sum_{l=0}^{N-1} \sum_{m=0}^{N-1} E\left\{n_{l} n_{m}^{*}\right\} e^{-j 2 \pi k m / N} e^{+2 \pi k m / N} \\
=\left[1+\cos \left(\phi_{k}\right)\right] \sum_{l=0}^{N-1} \sum_{m=0}^{N-1} E\left\{n_{l} n_{m}^{*}\right\} e^{-j 2 \pi k(l-m) / N}
\end{gathered}
$$

Samples of AWGN at different time instants are IID Gaussian random variables with zero mean. Therefore, they are uncorrelated and the variance can be represented as

$$
\begin{aligned}
E\left\{n_{m} n_{p}^{*}\right\} & = \begin{cases}E\left\{n_{m}\right\} E\left\{n_{p}^{*}\right\} & \text { if } m \neq p \\
E\left\{\left|n_{m}\right|^{2}\right\} & \text { if } m=p\end{cases} \\
& = \begin{cases}0 & \text { if } m \neq p \\
\sigma_{n_{m}}^{2} & \text { if } m=p\end{cases}
\end{aligned}
$$

Substituting Equation (5.33) into Equation (5.32) gives 


$$
\begin{aligned}
& \sigma_{\zeta}^{2}= {\left[1+\cos \left(\phi_{k}\right)\right] \sum_{m=0}^{N-1} E\left\{n_{m} n_{m}^{*}\right\} } \\
&=\left[1+\cos \left(\phi_{k}\right)\right] N \sigma_{n_{m}}^{2}
\end{aligned}
$$

where $\sigma_{n_{m}}^{2}$ is the noise power of the OFDM symbol in the time domain before the FFT operation. If correlation demodulator conditions are assumed, the noise power for OFDM symbol durations is given by

$$
\sigma_{n_{m}}^{2}=\frac{N_{0}}{2} \int_{-\infty}^{\infty}|H(f)|^{2} d f
$$

where $\frac{N_{0}}{2}$ (Watts per Hertz) is the power spectral density of the real part of noise and $H(f)$ is the frequency response of the integrator [48, 49]. Using Parsevel's theorem [52], Equation (5.35) converts to

$$
\begin{gathered}
\sigma_{n_{m}}^{2}=\frac{N_{0}}{2} \int_{0}^{T_{s}}|h[t]|^{2} d t \\
=\frac{N_{0}}{2} \int_{0}^{T_{s}} \frac{1}{T_{s}^{2}} d t \\
=\frac{N_{0}}{2 T_{s}}
\end{gathered}
$$

Substituting Equation (5.36) into Equation (5.34) gives

$$
\sigma_{\zeta}^{2}=\frac{\left[1+\cos \left(\phi_{k}\right)\right] N N_{0}}{2 T_{s}}
$$

Substituting, $T_{s}$ from Equation (3.33) and since for BPSK $T_{s}^{\prime}=T_{b}$, Equation (5.37) yields

$$
\sigma_{\zeta}^{2}=\frac{\left[1+\cos \left(\phi_{k}\right)\right] N N_{0}}{2 N b}=\sigma_{\zeta}^{2}=\frac{\left[1+\cos \left(\phi_{k}\right)\right] N_{0}}{2 T_{s}}
$$

Using Equations (5.24) and (5.38), the bit error probability $P_{b}^{\prime}$ conditioned on the value $\varphi_{k}$ can be expressed as

$$
P_{b}^{\prime}=\operatorname{Pr}\left\{\zeta_{k}<0 \mid 0 w a s \text { sent }\right\}
$$




$$
\begin{gathered}
=\operatorname{Pr}\left\{\frac{\zeta_{k}-\overline{\zeta_{k}^{+}}}{\sigma_{\zeta_{k}}}<\frac{\overline{\zeta_{k}^{+}}}{\sigma_{\zeta_{k}}} \mid " 0^{\prime \prime}\right\} \\
=\operatorname{Pr}\left\{\frac{\zeta_{k}-\overline{\zeta_{k}^{+}}}{\sigma_{\zeta_{k}}}>\frac{\overline{\zeta_{k}^{+}}}{\sigma_{\zeta_{k}}} \mid{ }^{\prime \prime}\right\} \\
=Q\left(\frac{\overline{\zeta_{k}^{+}}}{\sigma_{\zeta_{k}}}\right)=Q\left(\frac{2 A\left(1+\cos \left(\phi_{k}\right)\right)}{\sqrt{\left[1+\cos \left(\phi_{k}\right)\right] N_{0} / 2 T_{b}}}\right) \\
=Q\left(\frac{2 A \sqrt{T_{b}\left[1+\cos \left(\phi_{k}\right)\right]}}{\sqrt{N_{0}}}\right)=Q\left(2 \sqrt{\frac{E_{b}\left[1+\cos \left(\phi_{k}\right)\right]}{N_{0}}}\right)
\end{gathered}
$$

The number of data symbols per OFDM frame is 192 as discussed in Chapter III. The indices of these data symbols are given as $k=$ $9,10, \ldots, 100,156,157, \ldots, 255$. Thus, the average bit error probability $P_{b}$ is given by

$$
P_{b}=\frac{1}{192}\left(\sum_{k=9}^{100} Q\left(\sqrt{\frac{4\left[1+\cos \left(\phi_{k}\right)\right] E_{b}}{N_{0}}}\right)+\sum_{k=156}^{255} Q\left(\sqrt{\frac{4\left[1+\cos \left(\phi_{k}\right)\right] E_{b}}{N_{0}}}\right)\right)
$$

The simulation was conducted with increasing $E_{0} / N_{0}$ for 100,000 OFDM frames or 19.2 million data symbols per $E_{b} / N_{0}$ value. The simulated bit error rate (BER) for the MISO system is plotted in Figure 5.3 where $L=2$ is the number of transmit antennas and $J=1$ is the number of receive antennas. The theoretical probability of bit error as obtained in Equation (5.40) is also plotted. For comparison of performances, this figure also includes the theoretical probability of bit error for a baseband equivalent SISO system. The simulated results follow the theoretical results very closely. The MISO system with MDDM performs better than the SISO system for lower $E_{b} / N_{0}$ values and the performance of the MISO system is poorer than that of SISO system for $E_{b} / N_{0}$ greater than $6.5 \mathrm{~dB}$. 


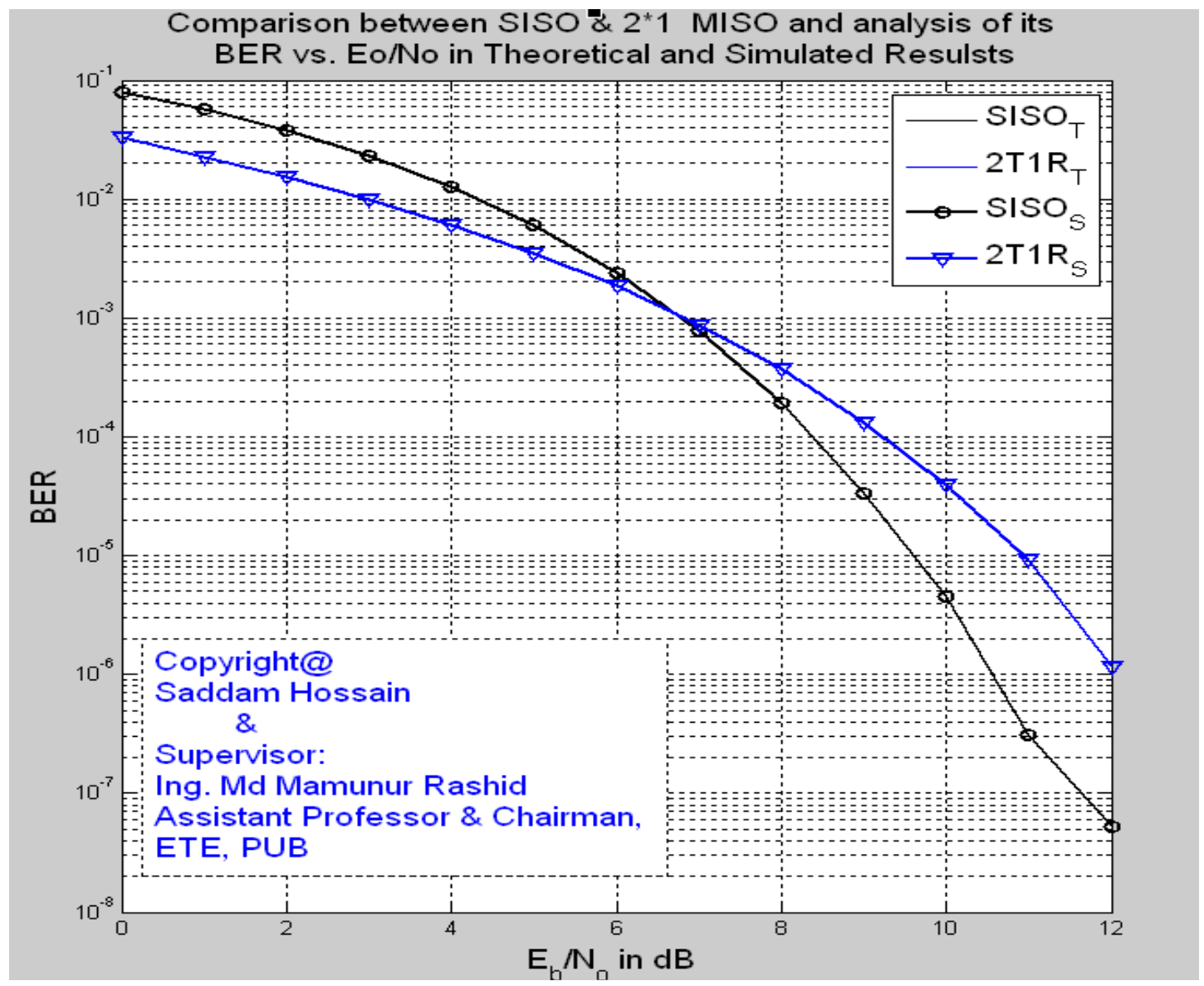

Figure 5.3: Simulation result of MDDM MISO system in AWGN

\subsubsection{Performance Analysis of MIMO System with Two Transmitting and Two Receiving Antennas}

For the MDDM MIMO system with two receive antennas, it is assumed that the signal receptions at both the antennas are uncorrelated and signals are received at the same time without any relative delay. The received signals at both receiving antennas are given by

$$
\begin{aligned}
& r_{m}^{1}=x_{m}^{1}+x_{m}^{2}+n_{m}^{1} \\
& r_{m}^{2}=x_{m}^{1}+x_{m}^{2}+n_{m}^{2}
\end{aligned}
$$

After the FFT operation the signals can be represented as

$$
\begin{aligned}
& R_{k}^{1}=X_{k}^{1}+X_{k}^{2}+N_{k}^{1} \\
& R_{k}^{2}=X_{k}^{1}+X_{k}^{2}+N_{k}^{2}
\end{aligned}
$$


Substituting Equation (3.31) and (3.19) into Equation (5.42) gives

$$
\begin{gathered}
R_{k}^{1}=X_{k}^{1}+X_{k}^{1} e^{-j 2 \pi k / N}+N_{k}^{1} \\
R_{k}^{2}=X_{k}^{1}+X_{k}^{1} e^{-j 2 \pi k / N}+N_{k}^{2} \\
R_{k}^{1}=\left(1+e^{-j \phi_{k}}\right) X_{k}^{1}+N_{k}^{1} \\
R_{k}^{2}=\left(1+e^{-j \phi_{k}}\right) X_{k}^{1}+N_{k}^{2}
\end{gathered}
$$

Following the derivation of Equation (5.23), Equation (5.43) converts to

$$
\begin{aligned}
& Z_{k}^{1}=R_{k}^{1}\left(1+e^{+j \phi_{k}}\right)=\left[2+2 \cos \left(\phi_{k}\right)\right] X_{k}^{1}+\left(1+e^{+j \phi_{k}}\right) N_{k}^{1} \\
& Z_{k}^{2}=R_{k}^{2}\left(1+e^{+j \phi_{k}}\right)=\left[2+2 \cos \left(\phi_{k}\right)\right] X_{k}^{1}+\left(1+e^{+j \phi_{k}}\right) N_{k}^{2}
\end{aligned}
$$

Now both space diversity receptions are combined to form random variable $Z_{k}$ which is given by

$$
\begin{gathered}
Z_{k}=Z_{k}^{1}+Z_{k}^{2} \\
=\left[4+4 \cos \left(\phi_{k}\right)\right] X_{k}^{1}+\left(1+e^{+j \phi_{k}}\right) N_{k}^{1}+\left(1+e^{+j \phi_{k}}\right) N_{k}^{2} \\
=4\left[1+\cos \left(\phi_{k}\right)\right] \frac{A}{\sqrt{2}}+\left(1+e^{+j \phi_{k}}\right) N_{k}^{1}+\left(1+e^{+j \phi_{k}}\right) N_{k}^{2} \\
=2 \sqrt{2} A\left[1+\cos \left(\phi_{k}\right)\right]+\left(1+e^{+j \phi_{k}}\right) N_{k}^{1}+\left(1+e^{+j \phi_{k}}\right) N_{k}^{2}
\end{gathered}
$$

If correlation demodulator conditions are assumed, then

$$
\begin{gathered}
E\left\{Z_{k} \mid \text { "0" was transmitted }\right\}=E\left\{\frac{1}{T_{b}} \int_{0}^{T_{b}} Z_{k} d t\right\} \\
=2 \sqrt{2} A\left[1+\cos \left(\phi_{k}\right)\right]
\end{gathered}
$$

The variance of $\zeta_{k}=\operatorname{Re}\left\{Z_{k}\right\}$ is only due to the variance of real part of noise components [48, 49]. Noise components at both receive antennas are IID 
Gaussian random variables. Therefore the total variance of the sum of two noise components is the sum of their individual variances [51]. The total noise variance is given as

$$
\sigma_{\zeta_{k}}^{2}=\sigma_{\zeta_{k}^{1}}^{2}+\sigma_{\zeta_{k}^{2}}^{2}
$$

Where $\zeta_{k}^{1}=\operatorname{Re}\left\{Z_{k}^{1}\right\}$ and $\zeta_{k}^{2}=\operatorname{Re}\left\{Z_{k}^{2}\right\}$. Following the derivation of noise variance derived in Equation (5.37), Equation (5.47) is represented as

$$
\begin{gathered}
\sigma_{\zeta_{k}}^{2}=\frac{\left[1+\cos \left(\phi_{k}\right)\right] N_{0}}{2 T_{b}}+\frac{\left[1+\cos \left(\phi_{k}\right)\right] N_{0}}{2 T_{b}} \\
=\frac{\left[1+\cos \left(\phi_{k}\right)\right] N_{0}}{T_{b}}
\end{gathered}
$$

and $\sigma_{\zeta_{k}}$ is given as

$$
\sigma_{\zeta_{k}}=\sqrt{\frac{\left(1+\cos \left(\phi_{k}\right)\right) N_{0}}{T_{b}}}
$$

Following the derivation of Equation (5.39), the bit error probability $P_{b}^{\prime}$ conditioned on the value $\phi_{k}$ is given by

$$
\begin{aligned}
P_{b}^{\prime} & =Q\left(\frac{2 \sqrt{2}\left[1+\cos \left(\phi_{k}\right)\right] A}{\sqrt{\left[1+\cos \left(\phi_{k}\right)\right] N_{0} / T_{b}}}\right) \\
& =Q\left(\sqrt{\frac{8\left[1+\cos \left(\phi_{k}\right)\right] A^{2} T_{b}}{N_{0}}}\right) \\
& =Q\left(\sqrt{\frac{8\left[1+\cos \left(\phi_{k}\right)\right] E_{b}}{N_{0}}}\right)
\end{aligned}
$$

Following the derivation of Equation (5.40) the average probability of bit error $P_{b}$ for a MDDM MIMO system with two transmit and two receiving antennas is given by 


$$
P_{b}=\frac{1}{192}\left(\sum_{k=9}^{100} Q\left(\sqrt{\frac{8\left[1+\cos \left(\phi_{k}\right)\right] E_{b}}{N_{0}}}\right)+\sum_{k=156}^{255} Q\left(\sqrt{\frac{8\left[1+\cos \left(\phi_{k}\right)\right] E_{b}}{N_{0}}}\right)\right)
$$

The simulation was conducted for the same number of OFDM frames or data symbols as mentioned in the previous section. The simulated bit error rate (BER) for the MIMO system is plotted in Figure 5.4. The simulated results follow theoretical results very closely.

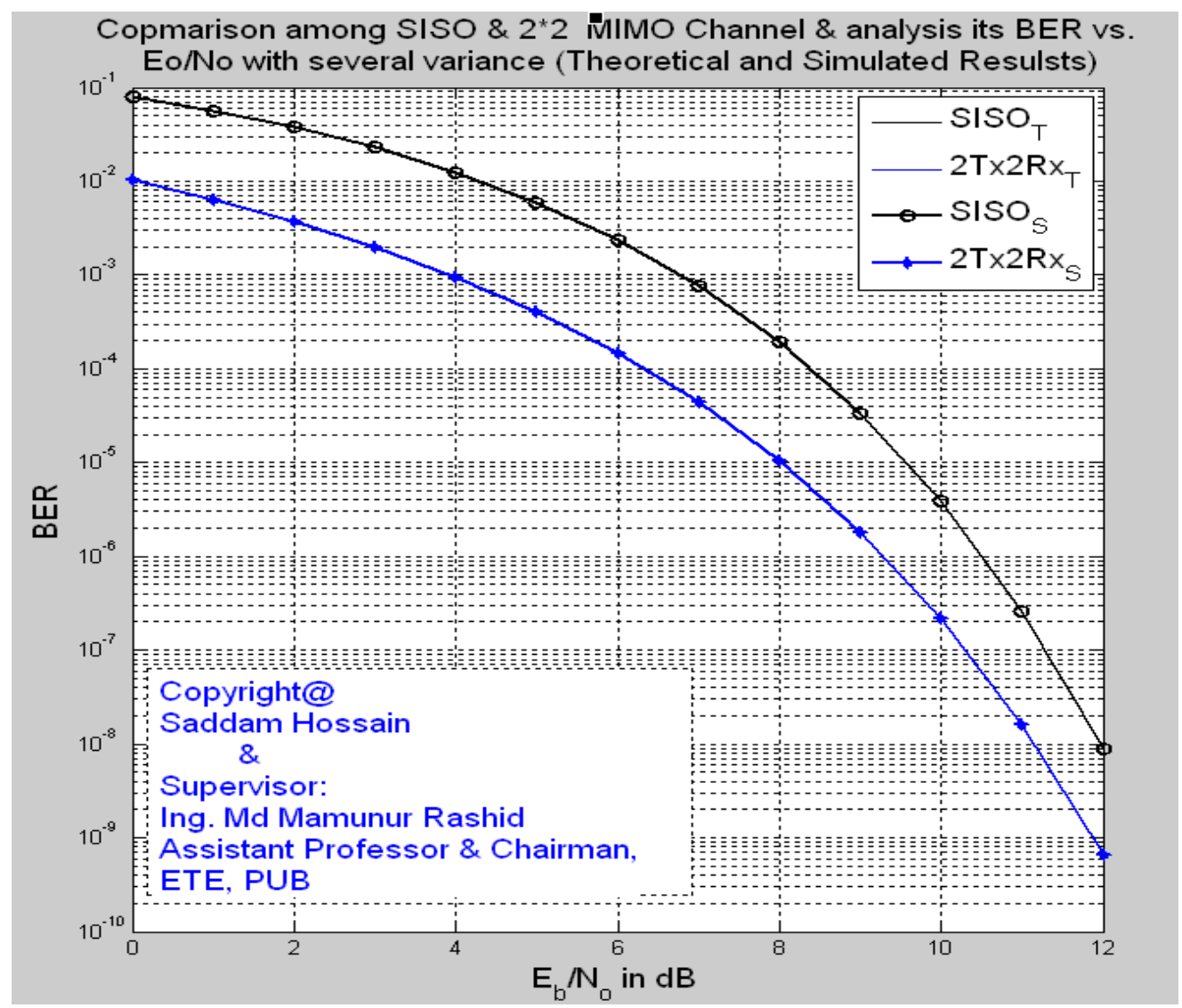

Figure 5.4: Simulation result of MDDM-2*2MIMO system in AWGN

\subsubsection{Performance Analysis of MIMO System with Two Transmitting and Three Receiving Antennas}

Similarly, in this section, the performance analysis of MDDM MIMO system with two transmit and three receive antennas is discussed. Exploiting the symmetry of the receiver design, the derivation of results is mainly based on the results presented in the last two sections. It is also assumed that the signal receptions at all antennas are uncorrelated and signals are received at the same time without any relative delay. The received signals at the three receive antennas are given by 


$$
\begin{gathered}
r_{m}^{1}=x_{m}^{1}+x_{m}^{2}+n_{m}^{1} \\
r_{m}^{2}=x_{m}^{1}+x_{m}^{2}+n_{m}^{2} \\
r_{m}^{3}=x_{m}^{1}+x_{m}^{2}+n_{m}^{3}
\end{gathered}
$$

After the FFT operation the signals are represented as

$$
\begin{gathered}
R_{k}^{1}=X_{k}^{1}+X_{k}^{2}+N_{k}^{1} \\
R_{k}^{2}=X_{k}^{1}+X_{k}^{2}+N_{k}^{2} \\
R_{k}^{2}=X_{k}^{1}+X_{k}^{2}+N_{k}^{3}
\end{gathered}
$$

Based on the discussion in previous sections, the components of the variables from all the space diversity receptions are

$$
\begin{gathered}
Z_{k}^{1}=R_{k}^{1}\left(1+e^{+j \phi_{k}}\right)=\left[2+2 \cos \left(\phi_{k}\right)\right] X_{k}^{1}+\left(1+e^{+j \phi_{k}}\right) N_{k}^{1} \\
Z_{k}^{2}=R_{k}^{2}\left(1+e^{+j \phi_{k}}\right)=\left[2+2 \cos \left(\phi_{k}\right)\right] X_{k}^{1}+\left(1+e^{+j \phi_{k}}\right) N_{k}^{2} \\
Z_{k}^{3}=R_{k}^{3}\left(1+e^{+j \phi_{k}}\right)=\left[2+2 \cos \left(\phi_{k}\right)\right] X_{k}^{1}+\left(1+e^{+j \phi_{k}}\right) N_{k}^{3}
\end{gathered}
$$

Now all the space diversity receptions are combined to form random variable $Z_{k}$ which is given by

$$
\begin{gathered}
Z_{k}=Z_{k}^{1}+Z_{k}^{2}+Z_{k}^{3} \\
=6\left[1+\cos \left(\phi_{k}\right)\right] X_{k}^{1}+\left(1+e^{+j \phi_{k}}\right) N_{k}^{1}+\left(1+e^{+j \phi_{k}}\right) N_{k}^{2}+\left(1+e^{+j \phi_{k}}\right) N_{k}^{3}
\end{gathered}
$$

If correlation demodulator conditions are assumed, then

$$
\begin{gathered}
E\left\{Z_{k} \mid \text { "0"was transmitted }\right\}=E\left\{\frac { 1 } { T _ { b } } \int _ { 0 } ^ { T _ { b } } \left[2 \sqrt{2} A\left[1+\cos \left(\phi_{k}\right)\right]+\right.\right. \\
\left.\left.N_{k}\left(1+e^{+j \phi_{k}}\right)\right] d t\right\}(5.56) \\
=2 \sqrt{2} A\left[1+\cos \left(\phi_{k}\right)\right]
\end{gathered}
$$


The variance of $\zeta_{k}=\operatorname{Re}\left\{Z_{k}\right\}$ is only due to the variance of real part of noise components [48, 49]. Noise components at both receive antennas are IID Gaussian random variables. Therefore the total variance of the sum of two noise components is the sum of their individual variances [51]. The total noise variance is given as

$$
\sigma_{\zeta_{k}}^{2}=\sigma_{\zeta_{k}^{1}}^{2}+\sigma_{\zeta_{k}^{2}}^{2}+\sigma_{\zeta_{k}^{3}}^{2}
$$

Where $\zeta_{k}^{1}=\operatorname{Re}\left\{Z_{k}^{1}\right\} \quad, \quad \zeta_{k}^{2}=\operatorname{Re}\left\{Z_{k}^{2}\right\}$ and $\zeta_{k}^{3}=\operatorname{Re}\left\{Z_{k}^{3}\right\}$. Following the derivation of noise variance derived in Equation (5.37), Equation (5.47) is represented as

and $\sigma_{\zeta_{k}}$ is given as

$$
\sigma_{\zeta_{k}}^{2}=\frac{3\left[1+\cos \left(\phi_{k}\right)\right] N_{0}}{2 T_{b}}
$$

$$
\sigma_{\zeta_{k}}=\sqrt{\frac{3\left(1+\cos \left(\phi_{k}\right)\right) N_{0}}{2 T_{b}}}
$$

Following the derivation of Equation (5.39), the bit error probability $P_{b}^{\prime}$ conditioned on the value $\phi_{k}$ is given by

$$
\begin{aligned}
P_{b}^{\prime} & =Q\left(\frac{3 \sqrt{2}\left[1+\cos \left(\phi_{k}\right)\right] A}{\sqrt{3\left[1+\cos \left(\phi_{k}\right)\right] N_{0} / 2 T_{b}}}\right) \\
& =Q\left(\sqrt{\frac{12\left[1+\cos \left(\phi_{k}\right)\right] A^{2} T_{b}}{N_{0}}}\right) \\
& =Q\left(\sqrt{\frac{12\left[1+\cos \left(\phi_{k}\right)\right] E_{b}}{N_{0}}}\right)
\end{aligned}
$$

The average probability of bit error $P_{b}$ for this case can be obtained by

$$
P_{b}=\frac{1}{192}\left(\sum_{k=9}^{100} Q\left(\sqrt{\frac{12\left[1+\cos \left(\phi_{k}\right)\right] E_{b}}{N_{0}}}\right)+\sum_{k=156}^{255} Q\left(\sqrt{\frac{12\left[1+\cos \left(\phi_{k}\right)\right] E_{b}}{N_{0}}}\right)\right)
$$


The simulation was conducted for the same number of OFDM frames or data symbols as mentioned in the previous section. The simulated bit error rate (BER) for the MIMO system is plotted in Figure 5.5. The simulated results follow theoretical results very closely.

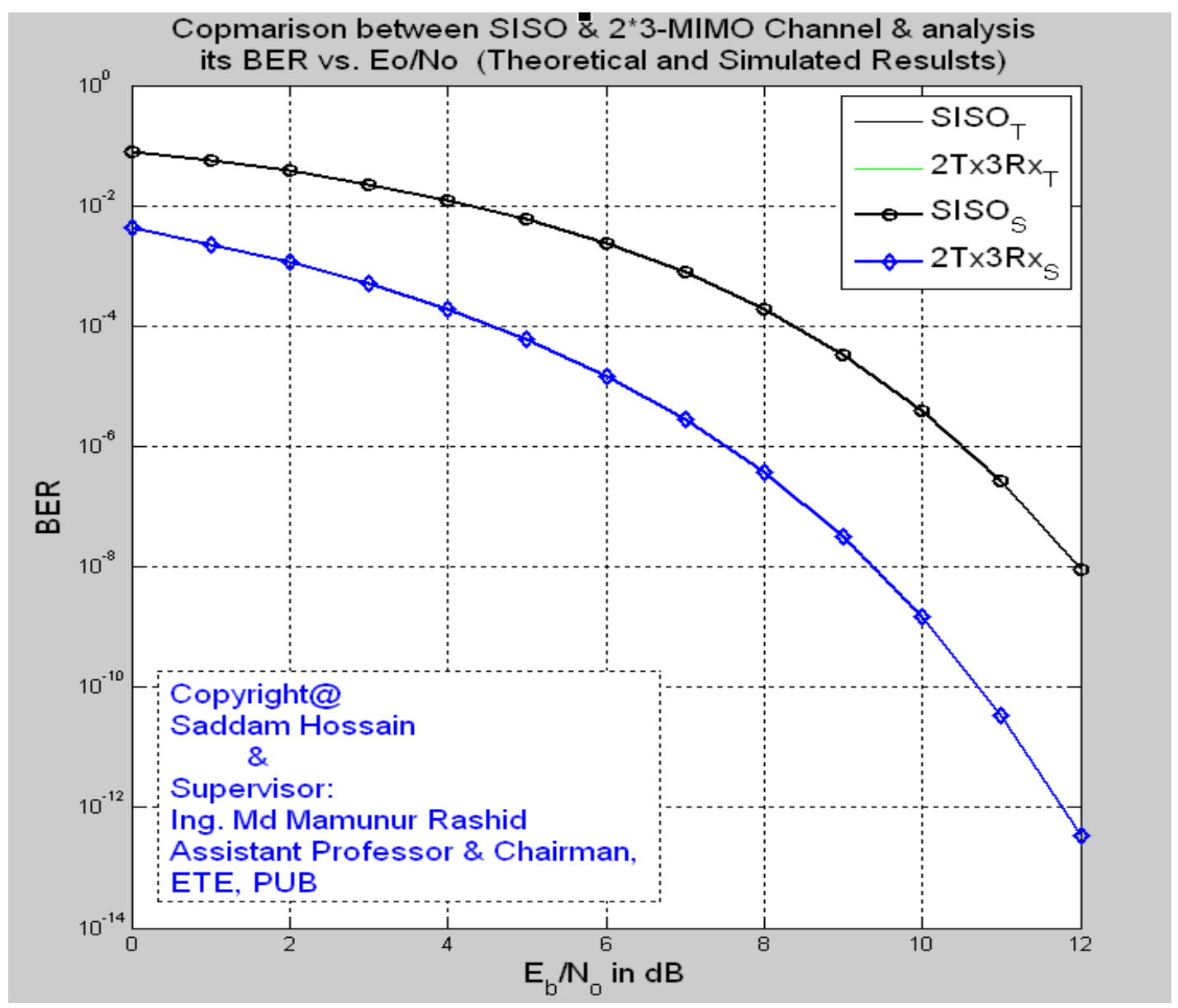

Figure 5.5: Simulation result of MDDM 2*3-MIMO system in AWGN

\subsubsection{Performance Analysis of MIMO System with Three Transmitting and Three Receiving Antennas}

Similarly, in this section, the performance analysis of MDDM MIMO system with three transmit and three receive antennas is discussed. Exploiting the symmetry of the receiver design, the derivation of results is mainly based on the results presented in the last two sections. It is also assumed that the signal receptions at all antennas are uncorrelated and signals are received at the same time without any relative delay. The received signals at the three receive antennas are given by 


$$
\begin{gathered}
r_{m}^{1}=x_{m}^{1}+x_{m}^{2}+x_{m}^{3}+n_{m}^{1} \\
r_{m}^{2}=x_{m}^{1}+x_{m}^{2}+x_{m}^{3}+n_{m}^{2} \\
r_{m}^{3}=x_{m}^{1}+x_{m}^{2}+x_{m}^{3}+n_{m}^{3}
\end{gathered}
$$

After the FFT operation the signals are represented as

$$
\begin{gathered}
R_{k}^{1}=X_{k}^{1}+X_{k}^{2}+X_{k}^{3}+N_{k}^{1} \\
R_{k}^{2}=X_{k}^{1}+X_{k}^{2}+X_{k}^{3}+N_{k}^{2} \\
R_{k}^{2}=X_{k}^{1}+X_{k}^{2}+X_{k}^{3}+N_{k}^{3}
\end{gathered}
$$

Based on the discussion in previous sections, the components of the variables from all the space diversity receptions are

$$
\begin{gathered}
Z_{k}^{1}=R_{k}^{1}\left(1+e^{+j \phi_{k}}\right)=\left[3+2 \cos \left(\phi_{k}\right)\right] X_{k}^{1}+\left(1+e^{+j \phi_{k}}\right) N_{k}^{1} \\
Z_{k}^{2}=R_{k}^{2}\left(1+e^{+j \phi_{k}}\right)=\left[3+2 \cos \left(\phi_{k}\right)\right] X_{k}^{1}+\left(1+e^{+j \phi_{k}}\right) N_{k}^{2} \\
Z_{k}^{3}=R_{k}^{3}\left(1+e^{+j \phi_{k}}\right)=\left[3+2 \cos \left(\phi_{k}\right)\right] X_{k}^{1}+\left(1+e^{+j \phi_{k}}\right) N_{k}^{3}
\end{gathered}
$$

Now all the space diversity receptions are combined to form random variable $Z_{k}$ which is given by

$$
\begin{gathered}
Z_{k}=Z_{k}^{1}+Z_{k}^{2}+Z_{k}^{3} \\
=9\left[1+\cos \left(\phi_{k}\right)\right] X_{k}^{1}+\left(1+e^{+j \phi_{k}}\right) N_{k}^{1}+\left(1+e^{+j \phi_{k}}\right) N_{k}^{2}+\left(1+e^{+j \phi_{k}}\right) N_{k}^{3}
\end{gathered}
$$

If correlation demodulator conditions are assumed, then

$$
\begin{gathered}
E\left\{Z_{k} \mid \text { "0"was transmitted }\right\}=E\left\{\frac { 1 } { T _ { b } } \int _ { 0 } ^ { T _ { b } } \left[3 \sqrt{2} A\left[1+\cos \left(\phi_{k}\right)\right]+\right.\right. \\
\left.\left.N_{k}\left(1+e^{+j \phi_{k}}\right)\right] d t\right\}(5.66) \\
=3 \sqrt{2} A\left[1+\cos \left(\phi_{k}\right)\right]
\end{gathered}
$$


The variance of $\zeta_{k}=\operatorname{Re}\left\{Z_{k}\right\}$ is only due to the variance of real part of noise components $[48,49]$. Noise components at both receive antennas are IID Gaussian random variables. Therefore the total variance of the sum of two noise components is the sum of their individual variances [51]. The total noise variance is given as

$$
\sigma_{\zeta_{k}}^{2}=\sigma_{\zeta_{k}^{1}}^{2}+\sigma_{\zeta_{k}^{2}}^{2}+\sigma_{\zeta_{k}^{3}}^{2}
$$

Where $\zeta_{k}^{1}=\operatorname{Re}\left\{Z_{k}^{1}\right\} \quad, \quad \zeta_{k}^{2}=\operatorname{Re}\left\{Z_{k}^{2}\right\}$ and $\zeta_{k}^{3}=\operatorname{Re}\left\{Z_{k}^{3}\right\}$. Following the derivation of noise variance derived in Equation (5.37), Equation (5.47) is represented as

and $\sigma_{\zeta_{k}}$ is given as

$$
\sigma_{\zeta_{k}}^{2}=\frac{3\left[1+\cos \left(\phi_{k}\right)\right] N_{0}}{2 T_{b}}
$$

$$
\sigma_{\zeta_{k}}=\sqrt{\frac{3\left(1+\cos \left(\phi_{k}\right)\right) N_{0}}{2 T_{b}}}
$$

Following the derivation of Equation (5.39), the bit error probability $P_{b}^{\prime}$ conditioned on the value $\phi_{k}$ is given by

$$
\begin{aligned}
P_{b}^{\prime} & =Q\left(\frac{3 \sqrt{2}\left[1+\cos \left(\phi_{k}\right)\right] A}{\sqrt{3\left[1+\cos \left(\phi_{k}\right)\right] N_{0} / 2 T_{b}}}\right) \\
& =Q\left(\sqrt{\frac{12\left[1+\cos \left(\phi_{k}\right)\right] A^{2} T_{b}}{N_{0}}}\right) \\
& =Q\left(\sqrt{\frac{12\left[1+\cos \left(\phi_{k}\right)\right] E_{b}}{N_{0}}}\right)
\end{aligned}
$$

The average probability of bit error $P_{b}$ for this case can be obtained by

$$
P_{b}=\frac{1}{192}\left(\sum_{k=9}^{100} Q\left(\sqrt{\frac{12\left[1+\cos \left(\phi_{k}\right)\right] E_{b}}{N_{0}}}\right)+\sum_{k=156}^{255} Q\left(\sqrt{\frac{12\left[1+\cos \left(\phi_{k}\right)\right] E_{b}}{N_{0}}}\right)\right)
$$


The simulation was conducted for the same number of OFDM frames or data symbols as mentioned in the previous section. The simulated bit error rate (BER) for the MIMO system is plotted in Figure 5.6. The simulated results follow theoretical results very closely.

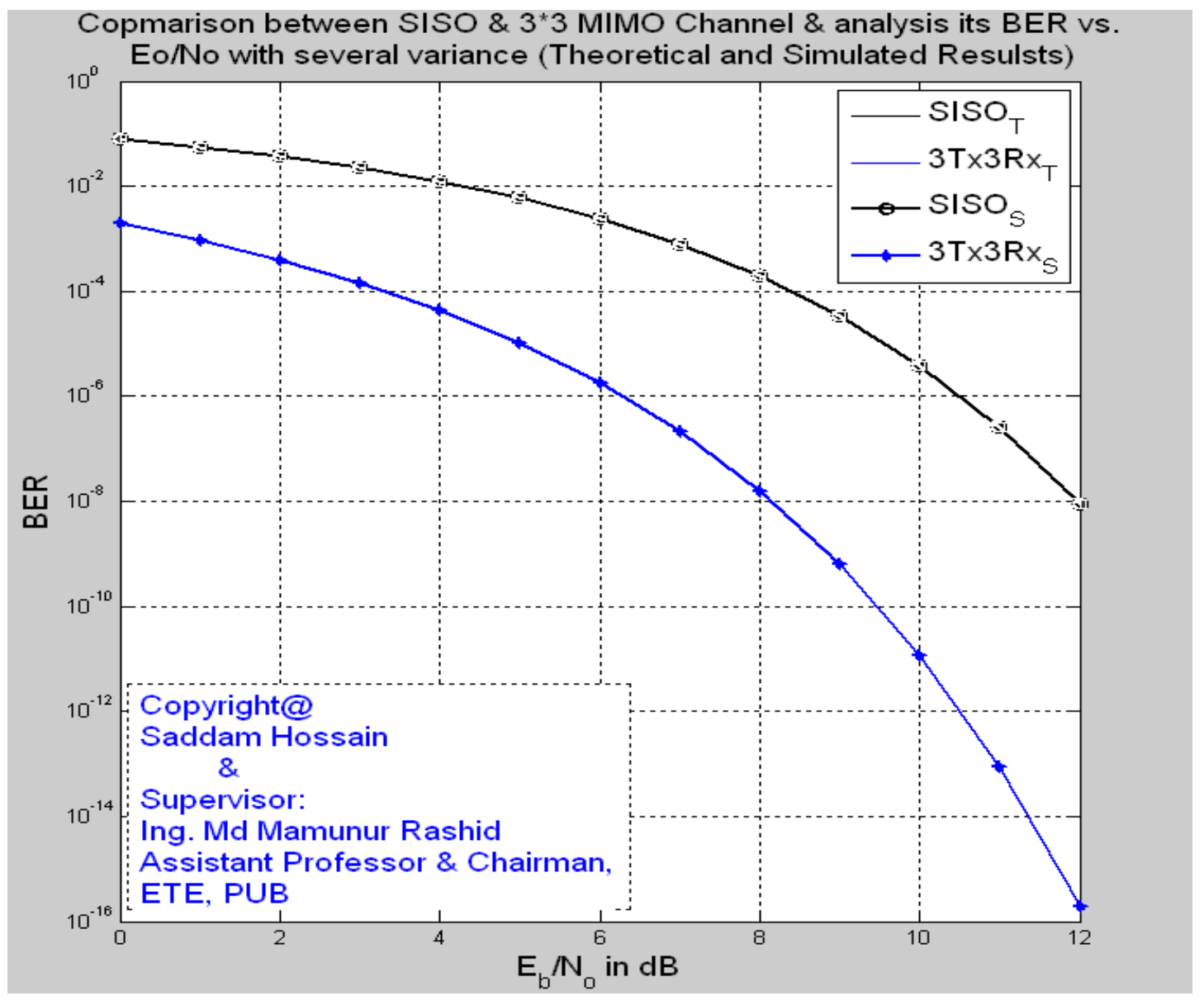

Figure 5.6: Simulation result of MDDM $3 * 3-M I M O$ system in AWGN

A comparison of Equations (5.40), (5.51) and (5.61) clearly reveals that the improvement factor increases linearly with increasing number of receiving antenna. The simulated bit error rate (BER) for the all MIMO systems are plotted in Figure 5.7.Thus, the average probability of bit error in AWGN channel for MDDM scheme discussed in this thesis with $J$ receiving antenna can be represented in general form as

$$
P_{b}=\frac{1}{192}\left(\sum_{k=9}^{100} Q\left(\sqrt{\frac{4 J\left[1+\cos \left(\phi_{k}\right)\right] E_{b}}{N_{0}}}\right)+\sum_{k=156}^{255} Q\left(\sqrt{\frac{4 J\left[1+\cos \left(\phi_{k}\right)\right] E_{b}}{N_{0}}}\right)\right)
$$




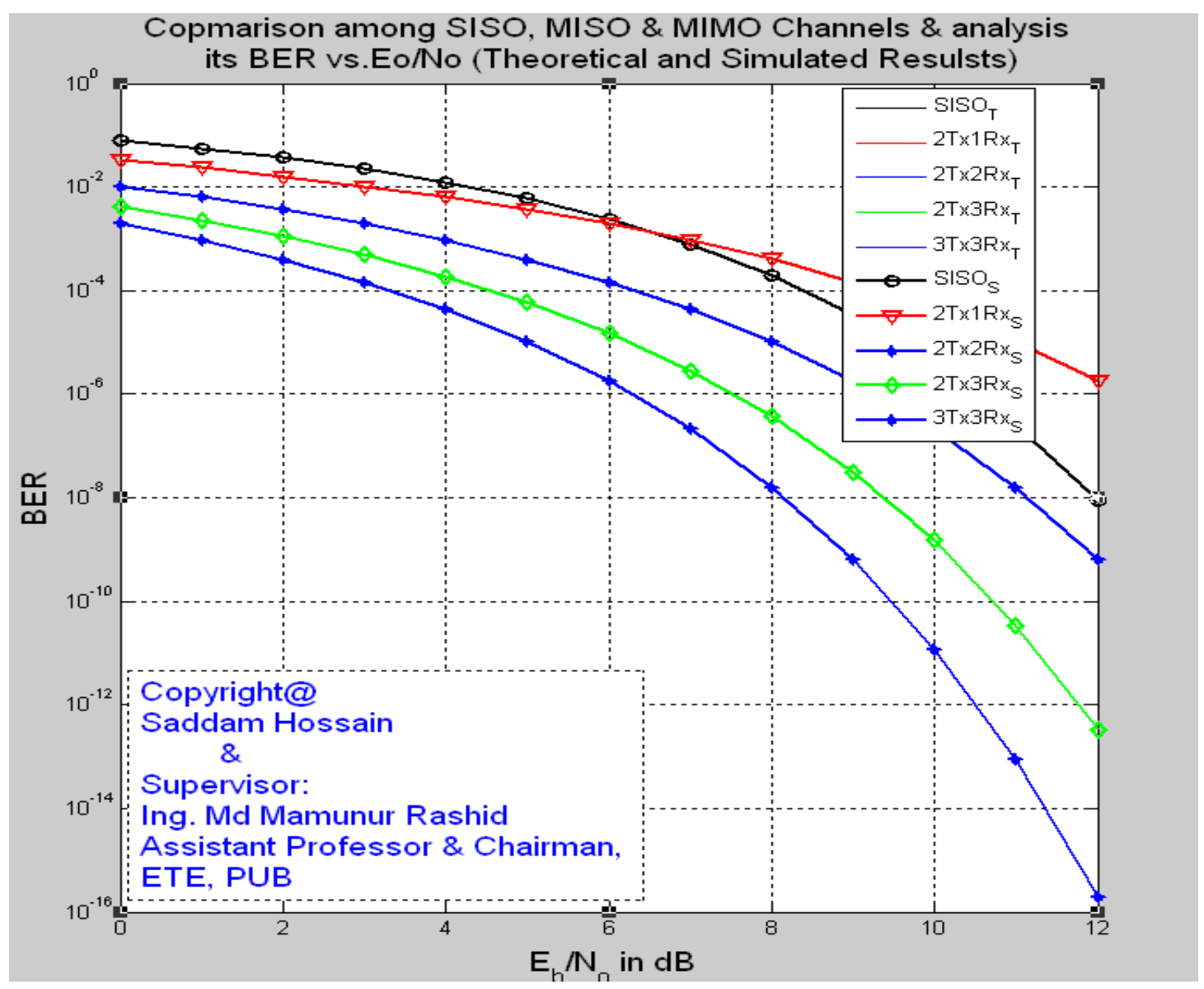

Figure 5.7: Simulation result of all MDDM MIMO systems in AWGN

\section{CONCLUSION}

The goal of this thesis was to design and analyze a MIMO system with multicarrier delay diversity modulation to mitigate BER. MDDM was incorporated with orthogonal frequency division multiplexing and cyclic delay diversity. A MISO system and MIMO systems were designed using the MDDM. They were simulated in MATLAB with BPSK and were tested in a progressive manner, in an AWGN channel. The BER performance of these systems was analyzed and compared with the performance of the simulated systems. The simulated performance results and theoretical analysis results were compared with the conventional SISO system performance. 


\section{APPENDIX}

\section{MATLAB CODES}

\section{This appendix includes the MATLAB code for simulation results}

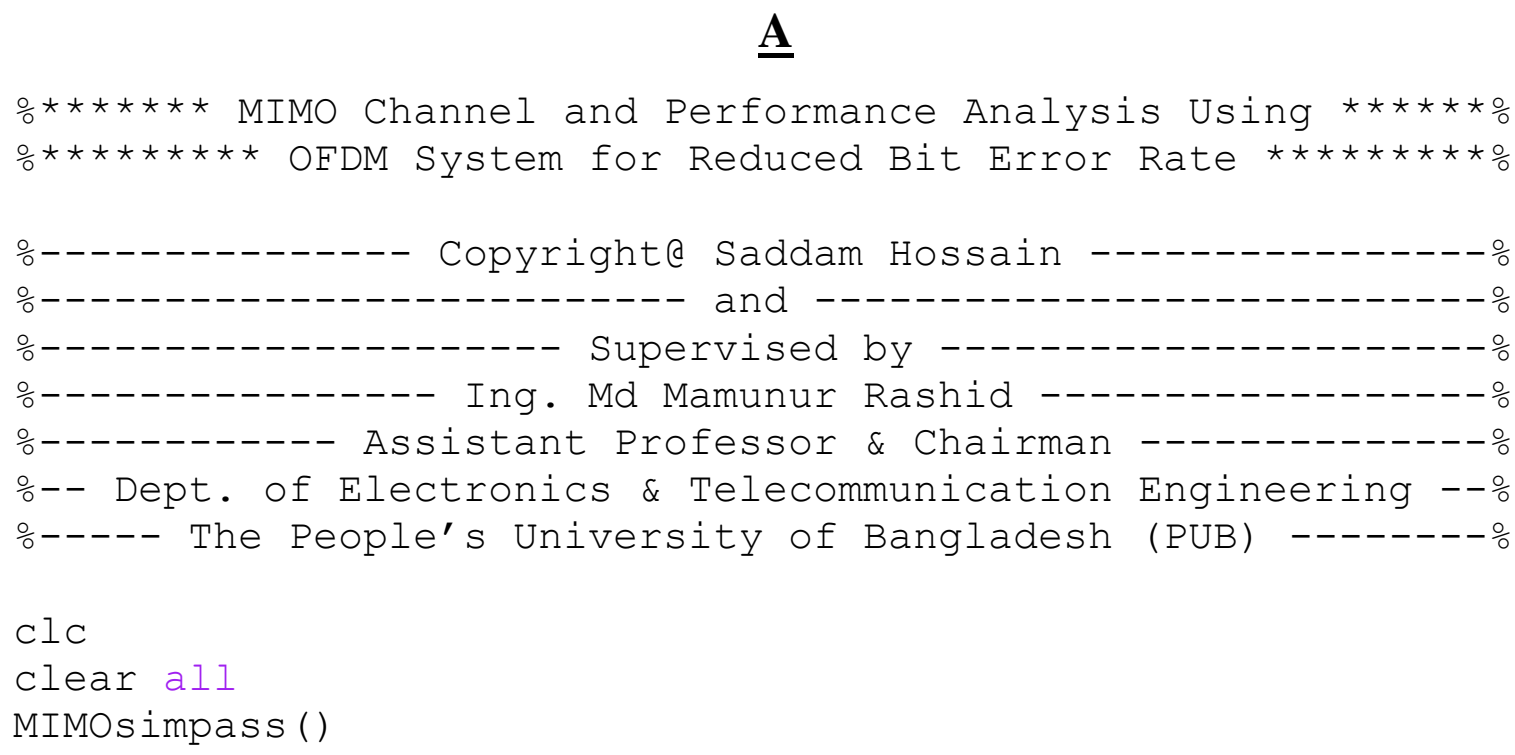

$\underline{\mathbf{A}}$

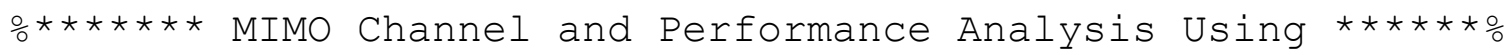

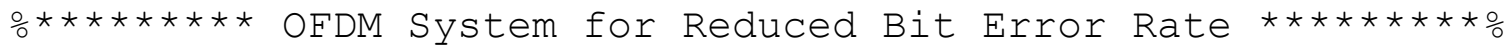




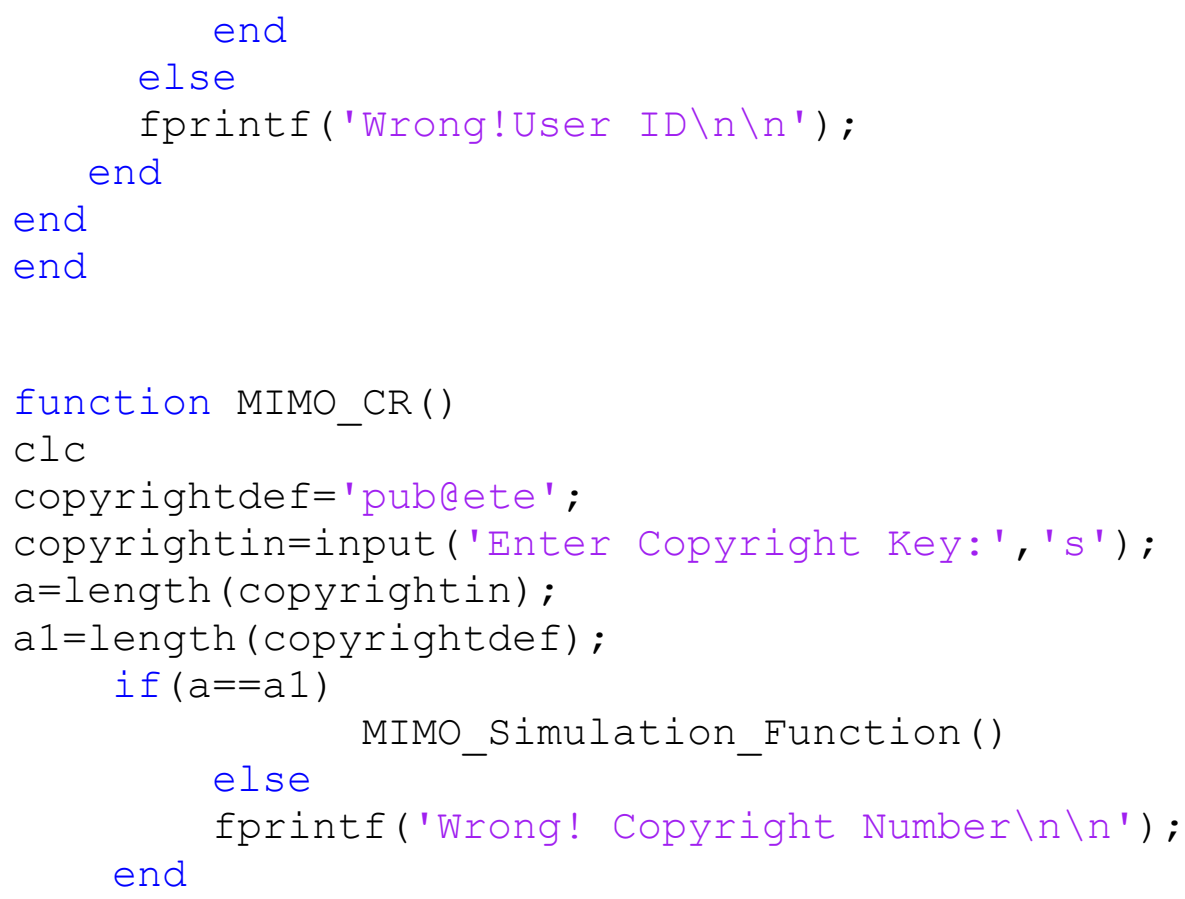




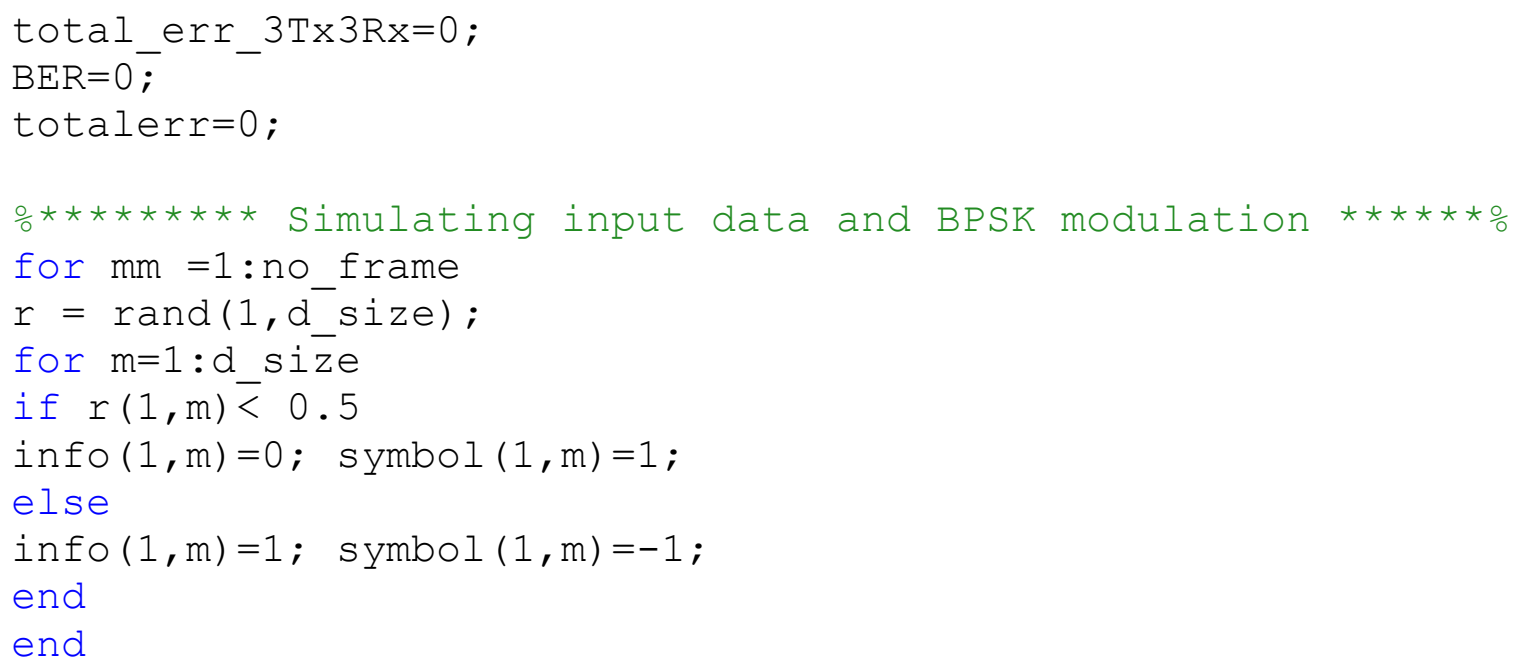

$\frac{0}{0} * * * * * * * * * * * * * * * * * * *$ Creating OFDMA Block $* * * * * * * * * * * * * * * * * \%$ Pre_IFFT_block $=[$ dc_comp pilot symbol (1:92)

zeros (1, (high_fc+low_fc)) symbol (93:d_size)];

응 $* * * * * * * * * * * * *$ Simulating Trnasmitted Signal $* * * * * * * * * * * * * * \circ$ txa_sigl = ifft((Pre_IFFT_block).'); \% taking IFFT after S/P coversion

txa_sig2 = circshift(txa_sig1.1); \% adding Cyclic Delay

Diversity

Txa_sig $=1 /$ sqrt $(2) *($ txa_sig1+txa_sig2) ;

o $* * * * * * * * * * * * * * * * * *$ Simulating Received signal $* * * * * * * * * * * * \frac{\circ}{0}$ Rxa_sig = awgn (symbol,SNR, 'measured'); Received signal for SISO BPSK

Rxa_sig_1 = awgn(Txa_sig,SNR, 'measured'); $\frac{\circ}{0}$ Received signal by Ânt. 1

Rxa_sig_2 = awgn(Txa_sig,SNR, 'measured'); Received signal by Ânt. 2

Rxa_sig_3 $=$ awgn(Txa_sig,SNR,'measured'); \% Received signal by Ant. 3

Rxa sig 4 = awgn(Txa sig, SNR, 'measured'); \% Received signal by Ant. 4

Rxa_2Tx1Rx $=$ fft $($ Rxa_sig_1); $\div 2$ Txa 1 Rxa Antennas

Rxa_2Tx2Rx $=$ fft $($ Rxa_sig_1+Rxa_sig_2); $\% 2$ Txa 2 Rxa

(Antennas)

Rxa_2Tx3Rx $=$ fft (Rxa_sig_1+Rxa_sig_2+Rxa_sig_3);2 Txa 3 Rxa (Antennas)

$\mathrm{Rxa} 3 \mathrm{Tx} 3 \mathrm{Rx}=$ fft $(\mathrm{Rxa}$ _sig_1+Rxa_sig_2+Rxa_sig_3+Rxa_sig_4); \% 3 Txa 3 Rxa (Antennas), Rxa_sing_4 can be Rxa_sig_3

Rxa_d_2Tx1Rx $=(\operatorname{Rxa} 2 \mathrm{Tx} 1 \mathrm{Rx} . *(1+$ phase_shift $))$; 


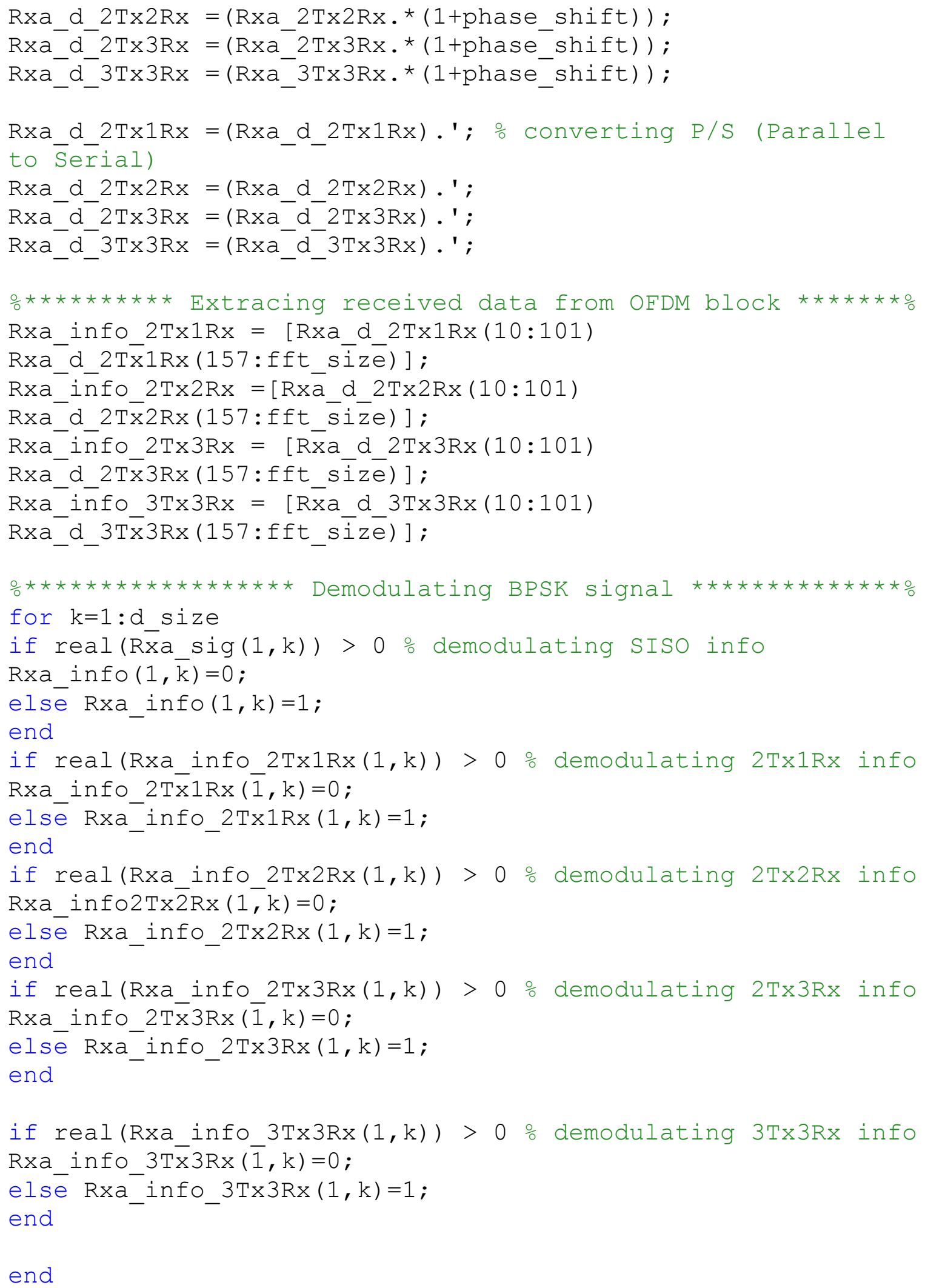

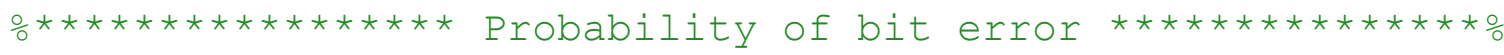




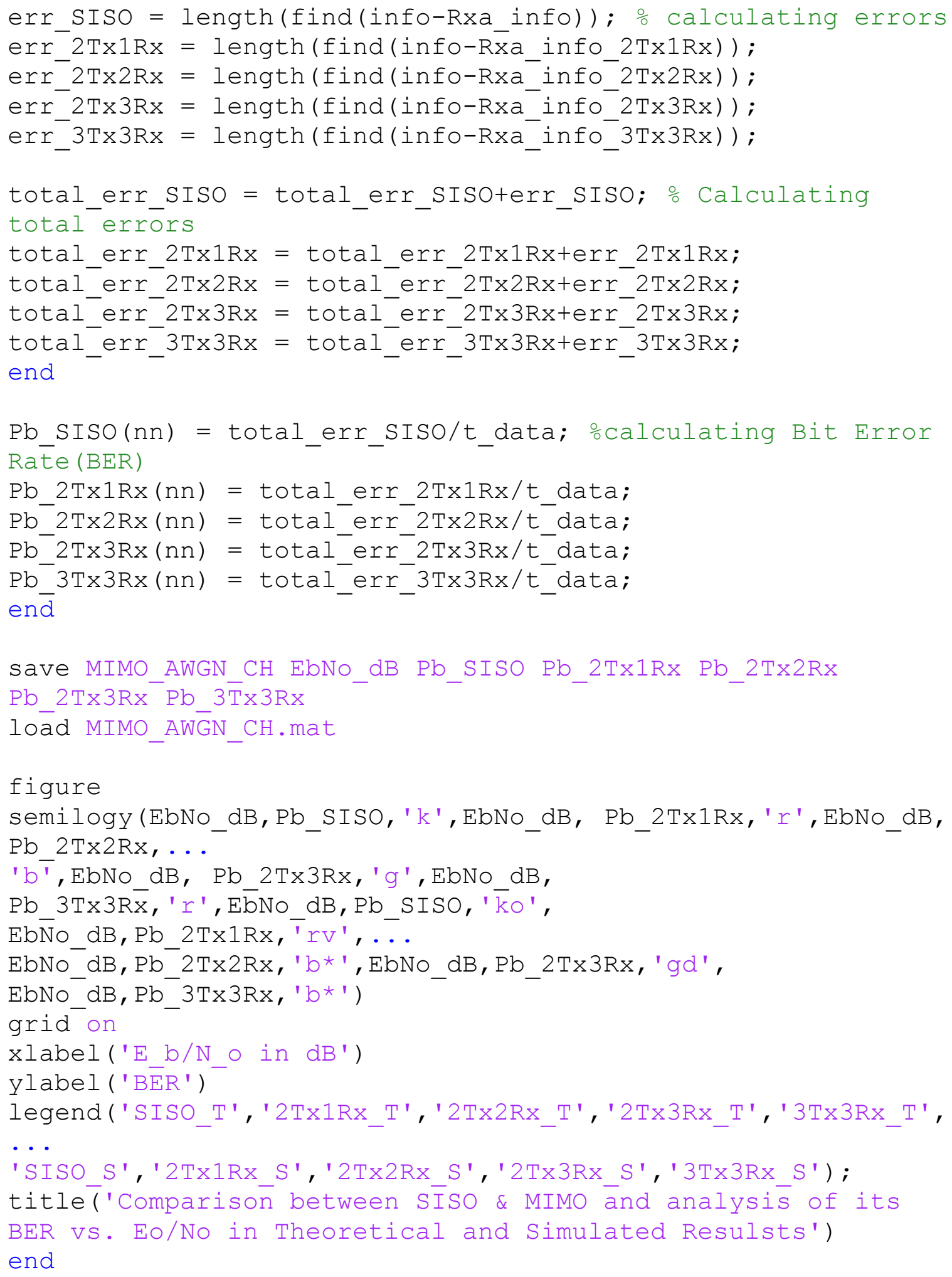


$\underline{\mathbf{B}}$

$\mathrm{ClC}$

clear all

MIMOsimpass ()

function MIMOsimpass()

$\mathrm{clc}$

userdef=' saddam' ;

passdef=' saddamhossain';

userin=input ('Enter Username: ', 's') ;

passin=input ('Enter Password: ', 's') ;

a=length (userin);

$a 1=$ length (userdef);

$\mathrm{b}=$ length (passin);

bl=length (passdef);

if $(\mathrm{a} \sim=\mathrm{a} 1 \quad \& \& \quad \mathrm{~b} \sim=\mathrm{b} 1)$

fprintf('Wrong! User ID and Password $\backslash n \backslash n$ ')

else

if $(a==a 1)$

if $(\mathrm{b}==\mathrm{b} 1)$

if (userin==userdef ) if (passin==passdef) MIMO_CR ( )

end

end

else

fprintf ('Wrong! Password \n\n') ; end

else

fprintf('Wrong! User ID $\backslash n \backslash n$ ') ;

end

end

end

function MIMO_CR()

$\mathrm{clc}$

copyrightdef= ' pubeete ' ;

copyrightin=input ('Enter Copyright Key: ', 's');

a=length (copyrightin) ;

al=length (copyrightdef) ;

if $(\mathrm{a}==\mathrm{a} 1)$

$$
\text { else }{ }^{\text {MIMO_Variance_Function( ) }}
$$




\section{end}

fprintf('Wrong! Copyright Number $\backslash n \backslash n$ ') ;

function MIMO_Variance_Function()

$\mathrm{clc}$

clear all

phase $=$ linspace $(0,2 *$ pi*255/256,256);

phi $=$ [phase $(10: 101)$ phase $(157: 256)]^{\prime}$;

complex $=(1+\cos (\mathrm{phi}))$;

EbNo_dB=0:12;

$\mathrm{EbNo}=10 . \wedge(\mathrm{EbNo} \mathrm{dB} / 10)$;

for $\mathrm{n}=1$ : length $(\mathrm{EbNO})$

sadd $=$ EbNo $(n)$;

for $k=1$ : length (phi)

$\operatorname{M} 2 \operatorname{Tx} 1 \operatorname{Rx}(k, n)=0.5 * \operatorname{erfc}(\operatorname{sqrt}(2 * \operatorname{sadd} * \operatorname{complex}(\mathrm{k})))$;

$\operatorname{M} 2 \operatorname{Tx} 2 \operatorname{Rx}(k, n)=0.5 * \operatorname{erfc}\left(\operatorname{sqrt}\left(4{ }^{*} \operatorname{sadd}{ }^{*} \operatorname{complex}(\mathrm{k})\right)\right)$;

$\operatorname{M} 2 \operatorname{Tx} 3 \operatorname{Rx}(k, n)=0.5 * \operatorname{erfc}\left(\operatorname{sqrt}\left(6{ }^{*} \operatorname{sadd}^{*} \operatorname{complex}(\mathrm{k})\right)\right)$;

$\operatorname{M3Tx} 3 \operatorname{Rx}(k, n)=0.5{ }^{*} \operatorname{erfc}\left(\operatorname{sqrt}\left(8{ }^{*} \operatorname{sadd}^{*} \operatorname{complex}(\mathrm{k})\right)\right)$;

end

end

응 $* * * * * * * * * * * * * * * * * * * * * *$ Theoratical BPSK $* * * * * * * * * * * * * * * * * \frac{\circ}{0}$

$\mathrm{Pb}$ SISO $=0.5 * \operatorname{erfC}(\operatorname{sqrt}(\mathrm{EbNO}))$;

$\mathrm{Pb}-2 \mathrm{Tx} 1 \mathrm{Rx}=1 /$ length $(\mathrm{phi}) * \operatorname{sum}(\mathrm{M} 2 \mathrm{Tx} 1 \mathrm{Rx})$;

$\mathrm{Pb} 2 \mathrm{Tx} 2 \mathrm{Rx}=1 /$ length (phi) $* \operatorname{sum}(\mathrm{M} 2 \mathrm{Tx} 2 \mathrm{Rx})$;

$\mathrm{Pb}_{-} 2 \mathrm{Tx} 3 \mathrm{Rx}=1 /$ length $(\mathrm{phi}) * \operatorname{sum}(\mathrm{M} 2 \mathrm{Tx} 3 \mathrm{Rx})$;

$\mathrm{Pb}_{-} 3 \mathrm{Tx} 3 \mathrm{Rx}=1 /$ length $(\mathrm{phi}) * \operatorname{sum}(\mathrm{M} 3 \mathrm{Tx} 3 \mathrm{Rx})$;

save Vari_AWGN_CH EbNo_dB Pb_SISO Pb_2Tx1Rx Pb_2Tx2Rx

$\mathrm{Pb} 2 \mathrm{Tx} 3 \mathrm{Rx}$ Pb_3Tx3Rx

loād Vari_AwḠ

$\frac{\circ}{0} * * * * * * * * * * * * * * *$ Plotting the simulation result $* * * * * * * * * * * * \frac{\circ}{0}$

figure

semilogy (EbNo_dB,Pb_SISO, 'k', EbNo_dB,

$\mathrm{Pb} 2 \mathrm{Tx} 1 \mathrm{Rx},{ }^{\prime} r$ ', $\mathrm{EbNo} \mathrm{dB}, \ldots$

$\mathrm{Pb} 2 \mathrm{Tx} 2 \mathrm{Rx},{ }^{\prime} \mathrm{b} \mathrm{\prime}^{\prime}, \mathrm{EbNo} \mathrm{dB}, \mathrm{Pb} 2 \mathrm{Tx} 3 \mathrm{Rx}$, ' $^{\prime}$ ',

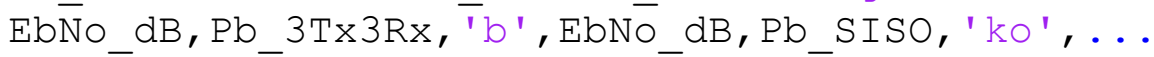

EbNo_dB, $\mathrm{Pb} 2 \mathrm{Tx} 1 \mathrm{Rx}, \mathrm{C}^{-} \mathrm{rV}$ ',

EbNo_dB, $\mathrm{Pb} \_2 \mathrm{Tx} 2 \mathrm{Rx}, \mathrm{b}^{-} \mathrm{b}$ ' ', EbNo_dB, $\mathrm{Pb} \_2 \mathrm{Tx} 3 \mathrm{Rx}, \mathrm{gd}^{\prime}$,

EbNo_dB, $\left.\mathrm{Pb} \_3 \mathrm{~T} \times 3 \mathrm{Rx}, \mathrm{b}^{\mathrm{b}} \mathrm{\prime}^{\prime}\right)$

grid on

xlabel ('E_b/N_o in $\mathrm{dB}$ ')

ylabel ( $\mathrm{BE} \overline{\mathrm{E}}$ ')

legend ('SISO_T', '2Tx1Rx_T', '2Tx2Rx_T', '2Tx3Rx_T', '3Tx3Rx_T',

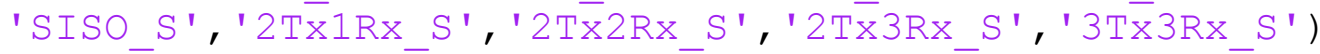


title('Copmarison between SISO and MIMO Channel \& analysis its BER vs. Eo/No with several variance (Theoretical and Simulated Resulsts)' )

end

\section{$\underline{\mathbf{C}}$}

oMimo Channel Capacity

clear all

clc

o Shannon capacity

$\sin =0$;

for $i=1: 10$

$\operatorname{snr}=\operatorname{snr}+2$;

$\mathrm{C}=\left(\log \left(1+10^{\wedge}(\operatorname{snr} / 10)\right)\right) / \log (2) ;$

$\mathrm{x}(\mathrm{i})=\operatorname{sn} r$;

$\mathrm{Y}(\mathrm{i})=\mathrm{C}$;

end

figure

plot (x,y, '- ', 'LineWidth' , 1.5)

hold on

o capacity of MIMO Link with $N R=2, N T=2$

$\mathrm{NR}=2$;

rand ('state', 456321)

$\sin =0$;

for $i=1: 10$;

$\operatorname{snr}=\operatorname{snr}+2$;

for $j=1: 10000$;

$c(j)=\left(N R \star \log \left(1+\left(10^{\wedge}(\operatorname{snr} / 10)\right) * \operatorname{abs}(\operatorname{normrnd}(0,1))\right) / \log (2)\right)$; end

yy $(i)=\operatorname{mean}(\mathrm{c})$;

$\mathrm{xx}(i)=\operatorname{snr}$;

end

plot (xx, yy, ' : ', 'Linewidth' ' 1.5)

o capacity of MIMO Link with $\mathrm{NR}=3, \mathrm{NT}=3$

$\mathrm{NR}=3$;

rand ('state', 456321)

$\operatorname{snr}=0$;

for $i=1: 10$;

$\operatorname{snr}=\operatorname{snr}+2$;

for $j=1: 10000$;

$c(j)=\left(N R * \log \left(1+\left(10^{\wedge}(\operatorname{snr} / 10)\right) * \operatorname{abs}(\operatorname{normrnd}(0,1))\right) / \log (2)\right)$; end 


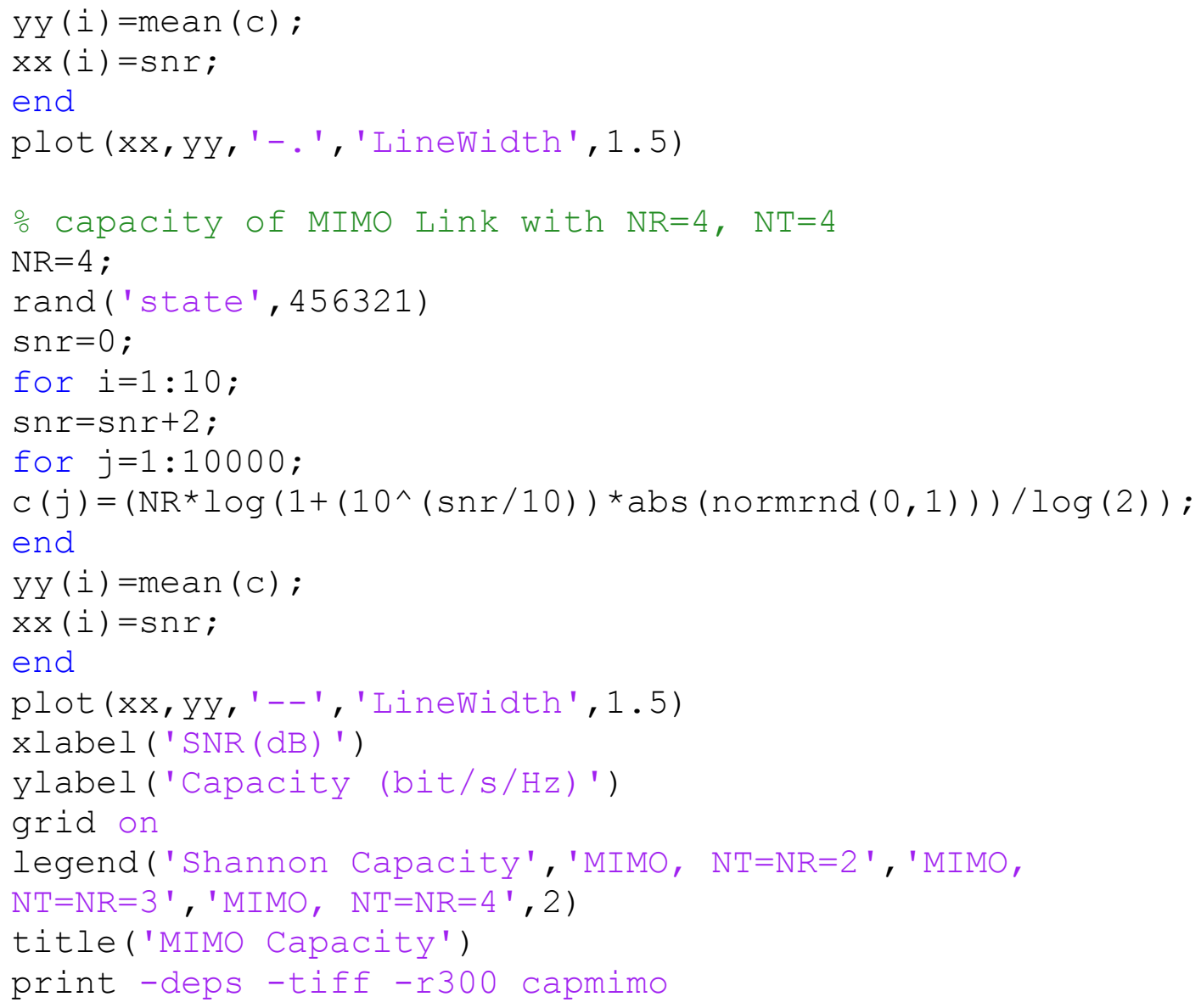




\section{REFERENCES}

[1] H. Sampath, S. Talwar, J. Tellado, V. Erce g and A. Paulraj," A Fourth-Generation MIMOOFDM Broadband Wireless System: Design, Performance, and Field Trial results, " IEEE Communications Magazine ,vol 40,no. 9,pp 143-149,September 2002

[2] Sam Jenkins "Implement OFDMA , MIMO for WiMAX ,LTE” picoChip , http://www.picochip.com/downloads/EEOL_2008MAR17_RFD_NETD_TA.pdf [10/11/08]

[3] M. Herdin, G. Gritsch, B. Badic and E Bonek, "The influence of channel models on simulated MIMO performance" 59th VTC 2004 Spring, Vol.1 pp 304 -307

[4] Saddam Hossain, "5G Wireless Communication Systems," American Journal of Engineering Research., vol. 02, Issue 10, pp. 344-353, Oct, 2013.

[5] D. Chizhik, G.J. Foschini, M.J. Gans, and R.A. Valenzuela,"Keyholes, correlations, and capacities of multielement transmit and receive antennas," IEEE Trans. on Wireless Commun., vol. 1, no. 2, pp. 361-368, Apr. 2002.

[6] M. Herdin, H. Özcelik, H. Hofstetter, and E. Bonek,"Linking reduction in measured MIMO capacity with dominant wave propagation ," in proc. of Int. Conf. on Telecommun., pp. 1526-1530, Mar. 2003.

[7] I. E. Telatar, "Capacity of multi-antenna gaussian channels," Office for Official Publications of the European Communities, AT\&T Bell Labs., Tech. Rep. BL0112170- 950615-07TM, 1995.

[8] K. Yu and B. E. Ottersten, "Models for MIMO propagation channels: a review." Wireless Communications and Mobile Computing, vol. 2, no. 7, pp. 653-666, 2002.

[9] T. Svantesson and A. Ranheim, "Mutual coupling effects on the capacity of multiple antenna systems," in Proc. IEEE ICASSP01, vol. 4, May 2001, pp. 24852488 .

[10] 3rd Generation Partnership Project (3GPP), "Spacial channel model for multiple input multiple output (MIMO) simulations (3gpp tr 25.996 version 6.1.0 release 6)," ETSI, Tech. Rep., 2003.

[11] W. C. Jakes, Microwave Mobile Communications New York: IEEE Press, 1993.

[12] K. Yu, M. Bengtsson, B. Ottersten, D. McNamara, P. Karlsson, and M. Beach,"Modeling of wideband MIMO radio channels based on NLOS indoor measurements," IEEE Trans. on Veh. Tech., vol. 53, no. 3, pp. 655-665, May 2004. 
[13] H. O־zcelik, M. Herdin, W. Weichselberger, J. Wallace, and E. Bonek, "Deficiencies of 'Kronecker' MIMO radio channel model," IEE Electronic Letters, vol. 39, no. 16, pp. 1209-1210, Aug. 2003.

[14] J. W. Wallace, and M. A. Jensen, "Modeling the indoor MIMO wireless channel,"IEEE Trans. Antennas Propagation, vol. 50, pp. 591-599, May 2002.

[15] A. Paulraj, R. Nabar, and D. Gore,"Introduction to space-time wireless communications," Cambridge, UK.: Cambridge Univ. Press, 2003

[16] T.D Abhayapala, T.S. Pollock, and R. Kennedy, "Spatial decomposition of MIMO wireless channels," in proc. of Int. Symp. on Sign. Proc. and its App , vol. 1, pp. 309-312, 2003.

[17] D. J. Cichon, and T. Kurner," Propagation Prediction Models", COST 231 Final Rep., 1995.

[18] M. F. Iskander, and Z. Yun, "Propagation prediction models for wireless communication systems," IEEE Trans. on Microw. The. and Tech., vol. 50, pp. 662-673, Mar 2002.

[19] F. Tila, P. R. Shepherd, and S. R. Pennock, "Theoretical capacity evaluation of indoor microand macro- MIMO systems at $5 \mathrm{GHz}$ using site specific ray tracing," IEE Electronics Letters, vol. 39, pp. 471-472, Mar. 2003.

[20] A.L. Swindlehurst, G. German, J. Wallace, and M. Jensen, "Experimental measurements of capacity for MIMO indoor wireless channels," in proc. of IEEE 3rdWorkshop on Signal Processing Advances in Wireless Commun., pp. 30;33, Mar. 2001.

[21] H. Zhu, J. Takada, and T. Kobayashi, "The verification of a deterministic spatiotemporal channel modeling approach by applying a deconvolution technique in the measurement," in proc. of IEEE Veh. Tech. Conf., vol. 1, pp. 362-366, May 2001.

[22] R. Tingley, and K. Bahlavan, "A statistical model of space-time radio propagation in indoor environments," in proc. of IEEE Antennas and Propagation forWireless Commun., pp. 61-64, Nov. 2000.

[23] C. Oestges, V. Erceg, and A.J. Paulraj,"A physical scattering model for MIMO macrocellular broadband wireless channels," IEEE J. on Select Areas in Commun., vol. 21, pp. 721-729, Jun. 2003.

[24] G.L. Turin, F.D. Clapp, T.L. Johnston, B.F. Stephen, and D. Lavry,"A statistical model of urban multipath propagation," IEEE Trans. on Veh. Tech., vol. 21, pp. 19, Feb. 1972. 
[25] R. J. C. Bultitude, S. A. Mahmoud, and W. A. Sullivan,“ A comparison of indoor radio propagation characteristics at $910 \mathrm{MHz}$ and $1.75 \mathrm{GHz}$," IEEE J. Select Areas Commun., vol. 7, pp. 20-30, Jan. 1989.

[26] L. M. Correia,"Wireless Flexible Personalised Communications," Wiley, 2001.

[27] J. Fuhl, A.F. Molisch, and E. Bonek, "Unified channel model for mobile radio systems with smart antennas," in proc. of Inst. Elect. Eng. Radar, Sonar and Navigation, vol. 145, pp. 32-41, Feb. 1998,

[28] Yong Soo Cho, Jaekwon Kim, Won Young Yang, Chung G. Kang," MIMOOFDM Wireless Communications With MATLAB," John Wiley \& Sons (Asia) Pte Ltd, 2 Clementi Loop, \# 02-01, Singapore 129809,

[29] Abdulla A. Abouda," Characterization of MIMO Channel Capacity in Urban Microcellular Environment," Helsinki University of Technology Communications Laboratory Technical Report T55 Teknillinen korkeakoulu Tietoliikennelaboratorio Raportti T55 Espoo 2007;

[30] C. Shannon, "A mathematical theory of communcation," Bell Labs Technical Journal, vol. 27, pp. 379-423,623-656, July and October 1948.

[31] T. S. Rappaport, Wireless Communications: Principles and Practice, 2nd ed. Prentice Hall, 2002.

[32] D. Gesbert, M. Shafi, D. shan Shiu, P. J. Smith, and A. Naguib, "From theory to practice: an overview of MIMO space-time coded wireless systems," IEEE J. Select. Areas Commun., vol. 21, no. 3, pp. 281-302, Apr. 2003.

[33] M. Stege, J. Jelitto, M. Bronzel, and G. Fettweis, “A multiple input-multiple output channel model for simulation of Tx-and Rx-diversity wireless systems," in proc. of IEEE Veh. Tech. Conf., vol. 2, pp. 833-839, Sep. 2000.

[34] Branka Vucetic and Jinhong Yuan, "Space-Time Coding." John Wiley \& Sons, West Sussex, England, 2003.

[35] Jun Tan, Gordon L. Stuber, "Multicarrier Delay Diversity Modulation for OFDM Systems." IEEE Transactions on Wireless Communications, Vol. 3, No. 5, September 2004. pp. 1756-1763.

[36] A. Wittneben, "A new bandwidth efficient transmit antenna modulation diversity scheme for linear digital modulation." in Proc. IEEE Int. Conf. Communications, 1993, pp. 1630-1634. 
[37] N. Seshadri and J. H. Winters, "Two signaling schemes for improving the error performance of frequency-division-duplex (FDD) transmission systems using transmitter antenna diversity." Int J. Wireless Inform. Networks, vol. 1, No. 1, pp. 24-47, January 1994.

[38] S. Kaiser, "Spatial transmit diversity techniques for broadband OFDM systems." in Proc. IEEE GLOBECOM, San Francisco, California, November 2000, pp. 18241828.

[39] A. Dammann and S. Kaiser, "Standard conformable antenna diversity techniques for OFDM and its application to the DVB-T system." in Proc. IEEE GLOBECOM, San Antonio, Texas, November 2001, pp. 3100-3105.

[40] A. Paulraj, R. Nabar and D. Gore, "Introduction to Space Time Wireless Communications." Cambridge University Press, Cambridge, United Kingdom, 2003.

[41] Michael J. Turpin, "An Investigation of a Multiple-Input Multiple-Output Communication System with the Alamouti Space-Time Code." Master's Thesis, Naval Postgraduate School, Monterey, California, June 2004.

[42] Erwin Kreyszig, "Advanced Engineering Mathematics, Fourth Edition." John Wiley \& Sons, New York, 1979.

[43] Bernard Sklar, "Digital Communications Fundamentals and Applications." Second Edition, Prentice Hall, Upper Saddle River, New Jersey, 2002.

[44] Meixia Tao and Toger S. Cheng, "Spade Code Design in Delay Diversity Transmission for PSK modulation." Vehicular Technology Conference, 200 Proceedings. IEEE 56th Volume 1, 24-28 Sept. 2002, pp. 444-448, vol. 1.

[45] Gerhaud Bauch and Javed Shamim Malik, "Orthogonal Frequency Division Multiple Access with Cyclic Delay Diversity.” ITG Workshop on Smart Antennas, 2004, pp. 17-24.

[46] Roberto Cristi, "Modern Digital Signal Processing." Brooks/Cole-Thomson Learning, Pacific Grove, California USA, 2004.

[47] Patrick A. Count, "Performance Analysis of OFDM in Frequency Selective, Slowly Fading Nakaghami Channel." Master's Thesis, Naval Postgraduate School, Monterey, California, June 2001.

[48] Clark Robertson, "EC4550 Digital Communications Systems Lecture Notes." Naval Postgraduate School, Monterey, California 2004 (unpublished). 
[49] John G. Proakis, "Digital Communications." Fourth Edition, McGraw Hill, New York, 2001.

[50] Theodore S. Rappaport, "Wireless Communications Principles and Practice." Second Edition, Prentice Hall PTR, Upper Saddle River, New Jersey, 2002.

[51] Charles W. Therrien, Murali Tummala, "Probability for Electrical and Computer Engineers.” CRC Press, Washington, D.C. 2004.

[52] Peyton Z. Peebles Jr., "Probability, Random Variables and Random Signal Principles." Fourth Edition, McGraw-Hill, New York, 2001.

ABC Research Alert, Vol 3, No 3 (2015)

ISSN 2413-5224 